Preprint typeset in JHEP style - HYPER VERSION

DESY 10-072

\title{
Reheating for closed string inflation
}

\author{
Michele Cicoli $^{1}$, and Anupam Mazumdar ${ }^{2,3}$ \\ ${ }^{1}$ Deutsches Elektronen-Synchrotron DESY, Notkestrasse 85, 22603 Hamburg, Germany \\ 2 Physics Department, Lancaster University, Lancaster, LA1 4YB, United Kingdom \\ 3 Niels Bohr Institute, Copenhagen University, Blegdamsvej-17, Copenhagen-2100, Denmark
}

ABSTRACT: We point out some of the outstanding challenges for embedding inflationary cosmology within string theory studying the process of reheating for models where the inflaton is a closed string mode parameterising the size of an internal cycle of the compactification manifold. A realistic model of inflation must explain the tiny perturbations in the cosmic microwave background radiation and also how to excite the ordinary matter degrees of freedom after inflation, required for the success of Big Bang Nucleosynthesis. We study these issues focusing on two promising inflationary models embedded in LARGE volume type IIB flux compactifications. We show that phenomenological requirements and consistency of the effective field theory treatment imply the presence at low energies of a hidden sector together with a visible sector, where the Minimal Supersymmetric Standard Model fields are residing. A detailed calculation of the inflaton coupling to the fields of the hidden sector, visible sector, and moduli sector, reveals that the inflaton fails to excite primarily the visible sector fields, instead hidden sector fields are excited copiously after the end of inflation. This sets severe constraints on hidden sector model building where the most promising scenario emerges as a pure $N=1 \mathrm{SYM}$ theory, forbidding the kinematical decay of the inflaton to the hidden sector. In this case it is possible to reheat the Universe with the visible degrees of freedom even though in some cases we discover a new tension between TeV scale SUSY and reheating on top of the well-known tension between TeV scale SUSY and inflation. 


\section{Contents}

1. Introduction 1

2. Inflation in the LARGE Volume Scenario

2.1 LARGE Volume Scenario

2.1.1 Geometric Regime 5

2.1.2 Quiver locus 6

2.2 Blow-up Inflation 7

2.2.1 Potential problems with string loops 8

2.3 Fibre Inflation 11

2.4 Moduli dynamics after inflation and reheating 13

3. Hidden sector dynamics

$3.1 N_{f}=0 \quad 16$

$3.2 N_{f}<\left(N_{c}-1\right)$

3.3 Constraint on hidden sector configurations 19

4. Inflaton couplings to hidden and visible sectors 20

4.1 Blow-up inflation 21

4.1.1 Geometric Regime 21

4.1 .2 Quiver Locus 27

4.2 Fibre Inflation 30

4.2.1 Geometric Regime 30

4.2 .2 Quiver Locus 33

5. Discussion and Conclusions 35

A. Calculation of the moduli couplings 37

A.1 Blow-up Inflation 37

A.1.1 Geometric regime 37

A.1.2 Quiver locus

A.2 Fibre Inflation 51

A.2.1 Canonical normalisation without loop corrections

A.2.2 Canonical normalisation with loop corrections 53

A.2.3 Moduli couplings 54

\section{Introduction}

Primordial inflation has been a very successful paradigm which can explain the observed temperature anisotropy in the cosmic microwave background (CMB) radiation [1] (for a recent review see [2]). Any successful model of inflation must also explain how to excite the Standard Model (SM) quarks and leptons after the end of inflation through the process of reheating (for a review see [3]) required for the success of Big Bang Nucleosynthesis [4. In this regard only visible sector models of inflation can be considered to be safe where the inflaton carries the SM charges 5 . Such models are primarily based on the Minimal Supersymmetric Standard Model (MSSM), where supersymmetry (SUSY) helps in obtaining a cosmologically flat potential via $D$-flat directions (for a review on MSSM flat directions see [6]). The advantage of visible sector models embedded within 
the MSSM is that it can also reproduce the required dark matter abundance necessary for the structure formation 7 .

On the contrary there exists a plethora of models of inflation where the inflaton belongs to the hidden sector since it is a SM gauge singlet. Typically these models can explain the temperature anisotropy in the CMB [2], but fail to explain how the inflaton energy density gets transferred primarily to visible sector degrees of freedom $(d o f)$, and not to hidden sector ones. Note that $a$ priori a gauge singlet inflaton has no preference to either the visible or the hidden sector. Therefore, it is a challenge to construct a model of inflation where the inflaton belongs to the hidden sector and motivate the inflaton couplings to hidden and visible $d o f$.

It is typical to encounter such a scenario in string theory which provides many closed string modes that are good inflaton candidates. In particular, the low energy effective action of string compactifications on Calabi-Yau three-folds typically has a large number of uncharged massless scalar fields with a flat potential, called moduli. These moduli can be the ideal candidates to drive inflation. Since their vacuum expectation value (VEV) determines various parameters (like masses and coupling constants) of the four-dimensional effective theory, it is important to stabilise them by providing masses to these moduli. Otherwise, the presence of these massless scalars with effective gravitational coupling would mediate unobserved long range fifth forces. It is well known by now that nonzero background fluxes induce potentials for some of the moduli [8, 9, 10]. However these fluxes severely backreact on the Calabi-Yau geometry generically modifying the internal space to a manifold with a more complicated structure. This is not the case for type IIB compactifications where the fluxes induce just a warp factor. For this reason, moduli stabilisation is best understood in the context of type IIB which is therefore well suited for the discussion of phenomenology and cosmology. However the stabilisation of all the geometric moduli requires to take into account various perturbative and non-perturbative effects [11, 12, 13].

One of the nice properties of type IIB flux compactifications is that global (or bulk) issues decouple from local (or brane) issues. The former are model independent issues which depend on moduli stabilisation and some of them are: (a) Different hierarchical scales, (b) SUSY breaking, (c) Soft SUSY breaking mass terms, (d) Cosmological constant, and (e) Inflation. On the other hand, the local issues depend on the particular local $D$-brane construction and some of them are: (a) Gauge group, (b) Chiral spectrum, (c) Yukawa couplings, (d) Gauge coupling unification, (e) Proton stability, and (f) Baryogenesis.

An excellent example of type IIB compactifications with stabilised moduli is given by the LARGE Volume Scenario (LVS) originally proposed in [12]. This is very appealing for particle physics phenomenology and cosmology. In these compactifications $\alpha^{\prime}$ and $g_{s}$ corrections can be combined with non-perturbative effects in order to generate a potential for all the Kähler moduli [13], whereas the background fluxes induce a potential for the dilaton and the complex structure moduli. Unlike the KKLT set-up [11], the moduli stabilisation is performed without fine tuning of the values of the internal fluxes and the Calabi-Yau volume is fixed at an exponentially large value (in string units). As a consequence, one has a very reliable four-dimensional effective description, as well as a tool for the generation of phenomenologically desirable hierarchies.

There are two different choices to embed the visible sector within LVS:

1. Geometric regime: the visible sector is built via magnetised intersecting $D 7$ branes wrapping a blow-up 4-cycle which is stabilised at a value larger than the string scale [14];

2. Quiver locus: the visible sector is built via fractional $D$-branes at the singularity obtained by shrinking down a blow-up mode 15.

Both of them have different phenomenology, the scales and hierarchies are different and we will briefly review them in the following section. 
One of the interesting features for all these setups is that at low energies they always come with a hidden and a visible sector. Therefore these models will serve as a perfect playground to study the inflaton couplings to both the dof. Furthermore, the two sectors do not have any direct coupling among themselves, except indirectly via the closed string sector. In this respect the two sectors are isolated from each other, nevertheless their total energy density will govern the dynamics of the Universe. It is then crucial to find an answer to each of the following questions: how does the inflaton couple to each sector? how does perturbative reheating take place? is it at all possible to excite predominantly the MSSM dof? Thankfully, these questions can be answered within this setup which involves an interesting interplay between local and global issues. We shall stress that reheating can set severe constraints both on the hidden sector physics and the scale of inflation which should be compatible with CMB predictions, successful reheating, and TeV scale SUSY ${ }^{1}$.

In order to study these issues, we shall focus on two very promising models of string inflation embedded in LVS, where the inflaton is a closed string mode, more precisely a Kähler modulus parameterising the size of an internal 4-cycle: (1) Blow-up inflation (BI) [20]: in this case the inflaton is the size of a blow-up mode yielding a small field inflationary model which is in good agreement with current observational data; and (2) Fibre inflation (FI) [21]: in this case the inflaton is the size of a $\mathrm{K} 3$ fibre over a $\mathbb{C} P^{1}$ base producing a large field inflationary model which can be a potential candidate if primordial gravity waves are discovered. These models represent some of the few known examples where inflation can be achieved without fine-tuning any parameter in the potential. In fact it is the typical no-scale structure of the potential that allows to solve the $\eta$-problem in a natural way.

In spite of the successes of these models we shall discover two outstanding challenges for closed string inflationary scenarios:

1. Most of the inflaton energy gets dumped to hidden sector $d o f$. This will lead to overproduction of hidden sector dark matter if there exists a stable species. This problem seems to rule out all the inflationary models unless the hidden sector consists just of an $N=1$ pure SYM theory. This severe constraint on hidden sector model building can be relaxed only for the geometric regime case with the inflaton wrapped by the visible sector $D 7$-stack.

2. Incompatibility between TeV scale SUSY and reheating on top of the well-know tension between inflation and TeV scale SUSY [22]: assuming that the density perturbations can be generated in a non-standard way by a second curvaton or modulating field [23], one could set the scale of the scalar potential in order to get inflation, the correct amount of CMB temperature fluctuations and TeV scale SUSY at the same time. However it might still be impossible to achieve an efficient reheating of the visible sector due to the fact that the inflaton might decay after BBN. This problem seems to rule out all the FI models (with the possible exception of $\mathrm{FI}$ at the quiver locus) and BI in the geometric regime with the inflaton not wrapped by the visible sector. The only models which are left over are BI in the geometric regime with the inflaton wrapped by the visible sector or BI at the quiver locus. It is important to stress that both these models require fine-tuning to have a successful inflationary scenario.

This paper is organised as follows. In section 2, we give a brief review of LVS stressing the different features of the geometric regime and the quiver locus case. We then discuss the dynamics of $\mathrm{BI}$ and FI presenting their predictions for the cosmological observables. We finally briefly describe the process of perturbative and non-perturbative reheating and thermalisation. In section 3, we

\footnotetext{
${ }^{1}$ Reheating and thermalisation have been studied previously in the context of closed string excitations in brane inflation 16], in the case of warped geometry 17, and in closed string models of inflation [18, 19]. In all these cases, no clear distinction has been made between hidden and visible dof. In this respect such studies are interesting but leave little impact on how the MSSM dof are excited, whose answer is relevant for the success of BBN.
} 
discuss the dynamics of the hidden sector and the constraints on hidden sector dof. In section 4 , we describe the inflaton couplings to both hidden and visible dof estimating the maximal reheating temperature in the approximation of sudden thermalisation of the visible sector for BI and FI, respectively. In section 5, we discuss our main results and conclude. In appendix A, we present a detailed derivation of the moduli canonical normalisation, mass spectrum, couplings and decay rates to any particle present in our models both for BI and FI. More precisely, we have derived the moduli couplings to fermions/sfermions, Higgs/Higgsinos, gauge bosons/gauginos of the visible and hidden sector, and the moduli self couplings.

\section{Inflation in the LARGE Volume Scenario}

In this section we shall present a brief review of the LVS and then proceed to describe the main features of the two inflationary models under consideration: blow-up and fibre inflation.

\subsection{LARGE Volume Scenario}

The LVS emerges naturally in the context of type IIB flux compactifications on Calabi-Yau orientifolds in the presence of space-time filling $D 3-D 7$ branes and $O 3-O 7$ planes. The overall volume of the Calabi-Yau is stabilised exponentially large by the interplay of non-perturbative and $\alpha^{\prime}$ corrections without fine-tuning the background fluxes [12]. Two big advantages of this scenario are that the effective field theory is under good control and one can generate hierarchies via the exponentially large volume. Expressing the Kähler moduli as $T_{i}=\tau_{i}+i b_{i}, i=1, \ldots, h_{1,1}$, with $\tau_{i}$ the volume of the internal 4-cycle $\Sigma_{i}$ and $b_{i}=\int_{\Sigma_{i}} C_{4}$, the only two conditions on an arbitrary Calabi-Yau to obtain the LVS are [13]:

1. $h_{1,2}>h_{1,1}>1$, where $h_{1,2}$ gives the number of complex structure moduli which are flux stabilised at tree-level.

2. The existence of at least one blow-up mode $\tau_{s}$ resolving a point-like singularity that gets non-perturbative corrections: $W=W_{0}+A_{s} e^{-a_{s} T_{s}}$. Then the overall volume can be fixed such that $\mathcal{V} \simeq \sqrt{\tau_{s}} e^{a_{s} \tau_{s}}$

The simplest examples of LVS are:

- Swiss-Cheese Calabi-Yau three-folds with volume given by [24]:

$$
\mathcal{V}=\alpha\left(\tau_{b}^{3 / 2}-\sum_{i} \gamma_{i} \tau_{i}^{3 / 2}\right)
$$

In this case all the $\tau_{i}$ are rigid cycles which are fixed small by non-perturbative corrections, whereas the large cycle $\tau_{b}$ is stabilised exponentially large due to $\alpha^{\prime}$ and non-perturbative effects.

- K3-fibered Calabi-Yau three-folds with volume of the form 25]:

$$
\mathcal{V}=\alpha\left(\sqrt{\tau_{1}} \tau_{2}-\sum_{i} \gamma_{i} \tau_{i}^{3 / 2}\right) .
$$

In this case still all the rigid cycles $\tau_{i}$ are fixed small by non-perturbative corrections, whereas the overall volume $\mathcal{V} \simeq \sqrt{\tau_{1}} \tau_{2}$ is stabilised exponentially large due to $\alpha^{\prime}$ and non-perturbative effects. The remaining flat direction can be frozen via the inclusion of subleading string loop corrections [26] which naturally fix both $\tau_{1}$ and $\tau_{2}$ large. 
Particular attention has to be paid on the stabilisation of the cycle supporting the visible sector which we shall call $\tau_{4}$ for later convenience in the notation. In fact $\tau_{4}$ has to be one of the small cycles in order not to get a visible gauge coupling $g^{-2} \sim \tau_{4}$ which is too small. However this cycle cannot get any non-perturbative correction since any instanton wrapped around $\tau_{4}$ will have chiral intersections with the visible sector inducing prefactors that depend on visible chiral fields 27:

$$
W_{n p} \supset\left(\Pi_{i} \Phi_{i}\right) e^{-a_{4} T_{4}}=0 \text { for }\left\langle\Phi_{i}\right\rangle=0 .
$$

Hence $\tau_{4}$ cannot be stabilised by non-perturbative effects but it can still be fixed by either $D$-terms [15, 25, 27, 28] or string loop corrections to the Kähler potential [13]. However $\tau_{4}$ can be stabilised either in the geometric regime, i.e. at values larger than the string scale, or it can shrink down at the singularity. These two regimes are characterised by different effective field theories with different features, which we discuss below.

\subsubsection{Geometric Regime}

In this case the cycle supporting the visible sector is stabilised in the geometric regime. There are two ways to do it:

1. via $D$-terms for intersecting rigid cycles [25, 27, 28].

2. via string loop corrections to the Kähler potential [13].

In this case the visible sector is built via magnetised intersecting $D 7$-branes wrapping $\tau_{4}$. Supersymmetry is broken due to non-vanishing background fluxes by the $F$-terms of the Kähler moduli [14. Hence we are in the presence of gravity, or better moduli-mediated supersymmetry breaking. In particular, the $F$-term of $\tau_{4}$ is non-zero:

$$
F^{4} \simeq e^{K / 2} K^{4 \overline{4}}\left(W_{\overline{4}}+W_{0} K_{\overline{4}}\right)=e^{K / 2} K^{4 \overline{4}} W_{0} K_{\overline{4}} \sim \frac{W_{0} \tau_{4}}{\mathcal{V}} \neq 0 .
$$

Then the main scales in the model are [14]:

- Planck-scale: $M_{P} \sim 10^{18} \mathrm{GeV}$.

- String-scale: $M_{s} \sim \frac{M_{P}}{\sqrt{\mathcal{V}}}$.

- Kaluza-Klein scale: $M_{K K} \sim \frac{M_{s}}{\tau_{4}^{1 / 4}} \sim \frac{M_{P}}{\tau_{4}^{1 / 4} \sqrt{\mathcal{V}}}$.

- Gravitino mass: $m_{3 / 2} \sim \frac{M_{P}}{\mathcal{V}}$.

- Soft-terms: $M_{\text {soft }} \sim m_{3 / 2}$.

- Small blow-up modes: $m_{\tau_{i}} \sim m_{3 / 2}$.

- Volume mode: $m_{\mathcal{V}} \sim \frac{M_{P}}{\mathcal{V}^{3 / 2}}$.

- Large mode orthogonal to the volume (only for K3-fibrations) [13: $m_{\chi} \sim \frac{M_{P}}{\mathcal{V}^{5 / 3}}$.

Setting the volume $\mathcal{V} \simeq 10^{14-15}$ in string units, corresponding to an intermediate $M_{s} \simeq 10^{11-12}$ $\mathrm{GeV}$, we have the following good phenomenological properties:

- TeV-scale SUSY: $M_{\text {soft }} \sim 1 \mathrm{TeV}$.

- Right QCD axion scale [29]: $f_{a} \sim M_{s} \sim 10^{11} \mathrm{GeV}$.

- Right Majorana scale for right handed neutrinos [30]: $M_{\nu_{R}} \sim M_{P} \mathcal{V}^{-1 / 3} \sim 10^{14} \mathrm{GeV}$, required for generating the observed light neutrino masses via the see-saw mechanism. 
We also point out that there are a few shortcomings:

1. No standard GUT theory since the scale for minimal $S U(5)$ or $S O(10)$ is much larger than the string scale.

2. No viable inflationary scenario given that BI works for $\mathcal{V} \simeq 10^{6-7}$, whereas FI works for $\mathcal{V} \simeq 10^{3-4}$. The latter scales are fixed by the observation of temperature anisotropies in the cosmic microwave background radiation [1].

3. Cosmological moduli problem for the volume mode (and $\chi$ in the case of compactifications on K3-fibered Calabi-Yau three-folds).

All these three problems could be solved at the same time by setting the volume smaller, of the order $\mathcal{V} \simeq 10^{4}$, and then fine-tuning the back-ground fluxes to give rise to a highly warped region where the visible sector sits. In this way $M_{\text {soft }}$ would redshift down to the $\mathrm{TeV}$ scale. However warping is usually negligible in constructions with exponentially large overall volume, and so this possibility does not seem very attractive.

As far as GUT theories are concerned, it is fair to say that there is no string model with $M_{s} \sim M_{G U T}$ which is able to reproduce the standard picture of gauge coupling unification, and so we do not consider LVS with an intermediate string scale less promising than other models in this regard. In addition there are left-right symmetric constructions where the gauge couplings unify at $10^{11-12} \mathrm{GeV}$ 31].

The authors of [32] proposed a model to reconcile the high scale of inflation with the lower scale of the soft SUSY breaking terms, by invoking inflation driven by the volume mode, even though this model requires a good amount of fine-tuning. Another way to get inflation and $\mathrm{TeV}$ scale SUSY at the same time, could be to generate the density perturbations via a non-standard mechanism, like a curvaton or a modulating field [23]. In this way, one might be able to lower the inflationary scale and solve, or more likely, attenuate this tension between cosmology and particle phenomenology. Finally it would be interesting to investigate if, in the case of compactifications with several moduli, the cosmological moduli problem could be solved due to the dilution factor induced by the out-of-equilibrium decay of a heavy modulus.

\subsubsection{Quiver locus}

When the cycle supporting the visible sector, $\tau_{4}$, is a rigid cycle which does not intersect with other cycles, it can shrink down at the singularity, $\tau_{4} \rightarrow 0$, due to $D$-terms, in the absence of visible sector singlets which can get a non-vanishing VEV 115.

In this case the visible sector is built via $D 3$-branes at the quiver locus. Supersymmetry is still broken due to non-vanishing background fluxes by the $F$-terms of the Kähler moduli which then mediate this breaking to the visible sector. However in this case the $F$-term of $\tau_{4}$ is vanishing:

$$
F^{4} \simeq e^{K / 2} K^{4 \overline{4}}\left(W_{\overline{4}}+W_{0} K_{\overline{4}}\right)=e^{K / 2} K^{4 \overline{4}} W_{0} K_{\overline{4}} \sim K^{4 \overline{4}} W_{0} \xi_{F I}=0,
$$

given that the $\tau_{4}$-cycle is stabilised by requiring a vanishing Fayet-Iliopoulos term $\xi_{F I}=0$. Therefore there is no local SUSY-breaking and the visible sector is sequestered. This implies that now the soft terms do not scale as the gravitino mass anymore but they can even be suppressed with respect to $m_{3 / 2}$ by an inverse power of the volume ${ }^{2}$. Then the main scales in the model are [15]:

- Planck-scale: $M_{P} \sim 10^{18} \mathrm{GeV}$.

- GUT-scale: $M_{G U T} \sim \mathcal{V}^{1 / 6} M_{s} \sim \frac{M_{P}}{\mathcal{V}^{1 / 3}}$.

\footnotetext{
${ }^{2}$ This might not be the case in the presence of a 1-loop redefinition of $\tau_{4}$ 33.
} 
- String-scale: $M_{s} \sim \frac{M_{P}}{\sqrt{\mathcal{V}}}$.

- Kaluza-Klein scale: $M_{K K} \sim \frac{M_{P}}{\mathcal{V}^{2 / 3}}$.

- Gravitino mass: $m_{3 / 2} \sim \frac{M_{P}}{\mathcal{V}}$.

- Small blow-up modes: $m_{\tau_{i}} \sim m_{3 / 2}$.

- Soft-terms: $M_{\text {soft }} \sim \frac{m_{3 / 2}^{2}}{M_{P}} \sim \frac{M_{P}}{\mathcal{V}^{2}}$.

- Volume mode: $m_{\mathcal{V}} \sim \frac{M_{P}}{\mathcal{V}^{3 / 2}}$.

- Large mode orthogonal to the volume (only for K3-fibrations): $m_{\chi} \sim \frac{M_{P}}{\mathcal{V}^{5 / 3}}$.

Setting the volume $\mathcal{V} \simeq 10^{6-7}$ in string units, corresponding to $M_{s} \simeq 10^{15} \mathrm{GeV}$, one finds several nice features [15]:

- Standard GUTs: $M_{G U T} \sim 10^{16} \mathrm{GeV}$.

- TeV scale SUSY: $M_{\text {soft }} \sim 1 \mathrm{TeV}$.

- No cosmological moduli problem as the mass of the moduli are larger than $10 \mathrm{TeV}$.

- Good inflationary model: BI.

We also point out that there are two shortcomings:

1. No correct size for the QCD axion decay constant.

2. No correct size for the Majorana mass term for right-handed neutrinos.

However these two seem not to be unsurmountable problems given that they might very well find viable solutions looking at open string modes as QCD axion candidates [34] or considering nonperturbative effects for the generation of neutrino masses [35].

For all these reasons the quiver locus seems more promising than the geometric regime. In this paper we shall investigate if this is still the case once we focus on the study of reheating of the MSSM dof after the end of inflation.

\subsection{Blow-up Inflation}

In this case the inflaton is the size of a blow-up mode [20]. The volume looks like:

$$
\mathcal{V}=\alpha\left(\tau_{1}^{3 / 2}-\gamma_{2} \tau_{2}^{3 / 2}-\gamma_{3} \tau_{3}^{3 / 2}-\gamma_{4} \tau_{4}^{3 / 2}\right),
$$

while the tree-level and non-perturbative superpotential takes the form:

$$
W=W_{0}+A_{2} e^{-a_{2} T_{2}}+A_{3} e^{-a_{3} T_{3}},
$$

and the tree-level Kähler potential with the leading order $\alpha^{\prime}$ correction reads:

$$
K=-2 \ln \left(\mathcal{V}+\frac{\xi}{2 g_{s}^{3 / 2}}\right), \quad \text { with } \quad \xi=\frac{\left(h_{1,2}-h_{1,1}\right) \zeta(3)}{(2 \pi)^{3}}>0 .
$$

The cycle $\tau_{4}$ is supporting the visible sector and so it does not get any non-perturbative correction. After minimising the axion directions and assuming that $\tau_{4}$ is fixed by either $D$-terms or $g_{s}$ corrections at the quiver locus or in the geometric regime, the supergravity $F$-term scalar potential is given by:

$$
V=\sum_{i=2}^{3} \frac{8 a_{i}^{2} A_{i}^{2}}{3 \alpha \gamma_{i}}\left(\frac{\sqrt{\tau_{i}}}{\mathcal{V}}\right) e^{-2 a_{i} \tau_{i}}-4 \sum_{i=2}^{3} W_{0} a_{i} A_{i}\left(\frac{\tau_{i}}{\mathcal{V}^{2}}\right) e^{-a_{i} \tau_{i}}+\frac{3 \xi W_{0}^{2}}{4 g_{s}^{3 / 2} \mathcal{V}^{3}}
$$


This potential completely stabilises $\tau_{2}, \tau_{3}$ and the volume $\mathcal{V} \simeq \alpha \tau_{1}^{3 / 2}$ at:

$$
a_{i}\left\langle\tau_{i}\right\rangle=\frac{1}{g_{s}}\left(\frac{\xi}{2 \alpha J}\right)^{\frac{2}{3}}, \quad\langle\mathcal{V}\rangle=\left(\frac{3 \alpha \gamma_{i}}{4 a_{i} A_{i}}\right) W_{0} \sqrt{\left\langle\tau_{i}\right\rangle} e^{a_{i}\left\langle\tau_{i}\right\rangle}, \quad \forall i=2,3,
$$

where $J=\sum_{i=2}^{3} \gamma_{i} / a_{i}^{3 / 2}$. Now displacing $\tau_{2}$ far from its minimum, due to the rapid exponential suppression, this blow-up mode experiences a very flat direction which is suitable for inflation. The reason why we have added the other blow-up mode $\tau_{3}$, which sits at its minimum while $\tau_{2}$ rolls down to the minimum, is to keep the volume minimum stable during inflation.

In terms of the canonically normalised inflaton $\phi$, the inflationary potential looks like [20]:

$$
V \simeq V_{0}-\beta\left(\frac{\phi}{\mathcal{V}}\right)^{4 / 3} e^{-a \mathcal{V}^{2 / 3} \phi^{4 / 3}}
$$

This is a model of small field inflation, and so no detectable gravity waves are produced during inflation: $r \equiv T / S \ll 1$. The spectral index turns out to be in good agreement with the observations: $0.960<n_{s}<0.967$, and the requirement of generating enough density perturbations fixes $\mathcal{V} \simeq$ $10^{6-7}$. This value of the volume is also the one preferred at the quiver locus to get both TeV scale SUSY and GUT theories. However for $\mathcal{V} \simeq 10^{6-7}$ the models in the geometric regime do not yield TeV scale SUSY. Hence this model of inflation seems to give a strong indication in favour of LVS at the quiver locus.

\subsubsection{Potential problems with string loops}

As we have seen in section 2.2, the inflationary potential is generated by tiny non-perturbative effects which make it naturally very flat. However potential dangerous problems can come from string loop corrections to the Kähler potential [26]. As pointed out in [13], due to the extended no-scale structure, these particular corrections are subleading in a large volume expansion with respect to the non-perturbative effects which fix the inflationary cycle $\tau_{2}$ small. However this is true only in a region in field-space around the LVS minimum, while once $\tau_{2}$ is displaced far from the minimum to drive inflation, the $g_{s}$ corrections quickly come to dominate the non-perturbative ones due to the rapid exponential suppression. Then these corrections spoil the flatness of the potential which is not suitable for inflation anymore. This can easily be seen from the form of the string loop corrections to the scalar potential in terms of the canonically normalised inflaton $\phi \sim \mathcal{V}^{-1 / 2} \tau_{2}^{3 / 4}$ :

$$
\delta V_{\left(g_{s}\right)}^{1-l o o p} \sim \frac{1}{\sqrt{\tau_{2}} \mathcal{V}^{3}} \sim \frac{1}{\phi^{2 / 3} \mathcal{V}^{10 / 3}}
$$

which induce a correction to the $\eta$-parameter of the form:

$$
\delta \eta \sim \frac{\partial^{2}\left(\delta V_{\left(g_{s}\right)}^{1-\text { loop }}\right)}{\partial \phi^{2}} \frac{\mathcal{V}^{3} g_{s}^{3 / 2}}{\xi} \sim \frac{g_{s}^{3 / 2}}{\phi^{8 / 3} \mathcal{V}^{1 / 3} \xi} \sim \frac{g_{s}^{3 / 2} \mathcal{V}}{\tau_{2}^{2} \xi} \gg 1
$$

In principle, there are two different way-outs to avoid this potential problem with string loop corrections:

1. Do not wrap any D7-brane around the inflationary 4-cycle;

2. Fine-tune the coefficients of the $g_{s}$ corrections.

Let us see these two cases in more detail. 


\section{- No D7 wrapped around the inflaton cycle}

If the inflaton 4-cycle is wrapped by an $E D 3$, the source of the string loop corrections is absent and the blow-up mode $\tau_{2}$ can safely drive inflation without any hidden sector located on it. However this is not possible for the following reason: denoting the inflaton 4-cycle as $\tau_{2}$ and the other blowup mode which keeps the volume minimum stable during inflation as $\tau_{3}$, the overall volume in the string frame scales as:

$$
\mathcal{V} \sim e^{\frac{a_{2} \tau_{2}}{g_{s}}} \sim e^{\frac{a_{3} \tau_{3}}{g_{s}}}
$$

where $a_{i}=2 \pi, i=2,3$, in the case of an $E D 3$, while $a_{i}=2 \pi / N$ in the case of an $S U(N)$ hidden sector that undergoes gaugino condensation. In addition both $\tau_{2}$ and $\tau_{3}$ has to be fixed larger than unity and $g_{s} \ll 1$ in order to trust the effective field theory. This implies that an ED3 can never yield $\mathcal{V} \simeq 10^{6-7}$ as it is needed to generate enough density perturbations. In fact, in the limiting case for $g_{s}=0.1$ and $\tau_{i}=1$, we already have:

$$
\mathcal{V} \sim e^{20 \pi} \sim 10^{27}
$$

and increasing $\tau_{i}$, the volume can just get larger. On the other hand, if $\tau_{i}$ is wrapped by $N=10$ $D 7$-branes, for $g_{s}=0.1$ and $\tau_{i}=2.6$, we obtain:

$$
\mathcal{V} \sim e^{\frac{20 \pi \tau_{i}}{N}} \sim e^{16.3} \sim 10^{7}
$$

Hence, in order not to fix any cycle supporting non-perturbative effects at a size smaller than the string scale, both $\tau_{2}$ and $\tau_{3}$ has to support a hidden sector that undergoes gaugino condensation. Thus we realise that hidden sectors are always present in these models, both in the geometric regime and at the quiver locus, and the only way to avoid problems with string loop corrections is to fine-tune their coefficient small. In addition the Hubble parameter during inflation $H_{\text {inf }}$ has always to be smaller than the gaugino condensation scale $\Lambda$ [36], and so one might be worried that at the beginning of inflation at large $\tau_{2}, \Lambda$ could turn out to be very low. However, in section of, we shall show that this is never the case throughout all the inflationary dynamics.

As we have already pointed out, the cycle supporting the visible sector, which we called $\tau_{4}$, cannot receive non-perturbative corrections since an instanton wrapped around $\tau_{4}$ will generically have chiral intersections with the visible sector. We expect that similar conclusions apply also to the case when the non-perturbative superpotential is generated via gaugino condensation since the presence of chiral matter generically forbids the emergence of such a condensate. Therefore these considerations seem to imply that the visible sector cannot be wrapped around $\tau_{2}$ resulting in an effective decoupling of the inflaton from the visible sector which can be very dangerous for reheating. However we could still envisage a quite generic situation where the visible sector $D 7$-stack wraps a combination of the cycles $\tau_{2}$ and $\tau_{4}$ such that the chiral intersections are with $\tau_{4}$ but not with $\tau_{2}$. In this set-up, one could still have non-perturbative corrections dependent on $\tau_{2}$ and, at the same time, the visible sector wrapped around $\tau_{2}$. Therefore in our study of reheating we shall consider two possible brane set-ups with the inflaton 4-cycle wrapped by both the visible sector and a hidden sector undergoing gaugino condensation or just by the hidden sector.

\section{- Fine-tuning the coefficient of the string loops}

The inflationary potential (2.9) takes the schematic form:

$$
V_{\left(n p+\alpha^{\prime}\right)}=\frac{\lambda_{1} \sqrt{\tau_{2}} e^{-2 a_{2} \tau_{2}}}{\mathcal{V}}-\frac{\lambda_{2} \tau_{2} e^{-a_{2} \tau_{2}}}{\mathcal{V}^{2}}+\frac{\lambda_{3} \sqrt{\tau_{3}} e^{-2 a_{3} \tau_{3}}}{\mathcal{V}}-\frac{\lambda_{4} \tau_{3} e^{-a_{3} \tau_{3}}}{\mathcal{V}^{2}}+\frac{\lambda_{5}}{g_{s}^{3 / 2} \mathcal{V}^{3}}
$$


Our aim is then to use $\tau_{2}$ as the inflationary direction, but we have now to worry about the string loop corrections which could spoil the flatness of this potential in the inflationary region, since they take the form 26:

$$
\delta V_{\left(g_{s}\right)}^{1-\text { loop }} \simeq \frac{\left(\mathcal{C}_{2}^{K K} g_{s}\right)^{2}}{\mathcal{V}^{3} \sqrt{\tau_{2}}}
$$

where we are assuming that the blow-up mode $\tau_{2}$ does not intersect with any other 4-cycle. Hence no winding corrections can be generated but only KK corrections coming from the exchange of Kaluza-Klein closed string modes between the $D 7$-brane wrapping $\tau_{2}$ and any other $D 7$ or $D 3$ brane present in the compactification.

Given that inflation takes place in the region of field space such that $a_{2} \tau_{2}>2 \ln \mathcal{V}$, the large exponential suppression in (2.17) tends to render the non-perturbative generated inflationary potential smaller than the loop generated potential (2.18) even though this is not the case close to the global minimum. Hence we need to fine tune $\mathcal{C}_{2}^{K K} \ll 1$ (by fine-tuning the complex structure moduli), and in order to understand the amount of fine-tuning needed to avoid this problem, we have to make sure that $V_{\left(g_{s}\right)}<V_{(n p)}$ for initial values of $\tau_{2}$ that give rise to around 60 e-foldings of inflation. The number of e-foldings is given by [20]:

$$
N_{e} \simeq \frac{1}{\mathcal{V}^{2}} \int_{2 \ln \mathcal{V}}^{n \ln \mathcal{V}} \frac{e^{a_{2} \tau_{2}}}{\sqrt{\tau_{2}}\left(a_{2} \tau_{2}-1\right)} d \tau_{2},
$$

while the ratio between $(2.17)$ and $(2.18)$ looks like (considering just the $\tau_{2}$-dependent bit of each expression):

$$
R \equiv \frac{V_{\left(g_{s}\right)}}{V_{(n p)}} \simeq \frac{\left(\mathcal{C}_{2}^{K K} g_{s}\right)^{2}}{\mathcal{V}} \frac{e^{a_{2} \tau_{2}}}{\lambda_{2} \tau_{2}^{3 / 2}}, \quad \text { with } \quad \lambda_{2} \simeq 10 .
$$

Then we need to impose that $R \ll 1$ at $a_{2} \tau_{2}=n \ln \mathcal{V}$ with $n$ such that $N_{e}=60$. Expressing the volume as $\mathcal{V}=10^{x}, \mathcal{C}_{2}^{K K} \simeq 10^{-y}$ and fixing the string coupling such that $g_{s}=0.1,{ }^{3}$ this corresponds to imposing:

$$
R \simeq \frac{10^{(n-1) x-2 y-3}}{x^{3 / 2}} \ll 1 .
$$

For each value of $x$, we can work out $n$ numerically from (2.19) and then, substituting these two values in (2.21), we derive the amount of fine-tuning $y$. The results are summarised in Table 1.

\begin{tabular}{|c|c|c|c|}
$x$ & $n$ & $y$ & $R$ \\
\hline \hline 4 & 3.03 & 2.5 & 0.16 \\
\hline 6 & 2.72 & 3.5 & 0.14 \\
\hline 8 & 2.56 & 4.5 & 0.13 \\
\hline 10 & 2.46 & 5.5 & 0.12 \\
\hline 12 & 2.39 & 6.5 & 0.11 \\
\hline 14 & 2.34 & 7.5 & 0.11
\end{tabular}

Table 1: Estimation of the fine-tuning of $\mathcal{C}_{2}^{K K}=10^{-y}$ needed to render the string loops subleading with respect to the inflationary potential. Here $\mathcal{V}=10^{x}$ and $n$ is such that $N_{e} \simeq 60$.

\footnotetext{
${ }^{3}$ We prefer to focus all the fine-tuning on $\mathcal{C}_{2}^{K K}$ preserving $g_{s}$ not too small since the volume of a 4 -cycle in the string frame is related to the same quantity in the Einstein frame as $\tau_{s}=g_{s} \tau_{E}$, and so a very small $g_{s}$ might lead $\tau_{s} \ll 1$ in the regime where we do not trust anymore the supergravity approximation.
} 


\subsection{Fibre Inflation}

In this case the inflaton is the size of a $K 3$ fibre over a $\mathbb{C} P^{1}$ base [21]. The volume looks like:

$$
\mathcal{V}=\alpha\left(\sqrt{\tau_{1}} \tau_{2}-\gamma_{3} \tau_{3}^{3 / 2}-\gamma_{4} \tau_{4}^{3 / 2}\right)
$$

while the superpotential takes the form ${ }^{4}$ :

$$
W=W_{0}+A_{3} e^{-a_{3} T_{3}},
$$

and the Kähler potential has the same form as in (2.8). The cycle $\tau_{4}$ is supporting the visible sector and so it does not get any non-perturbative correction. After minimising the axion directions and assuming that $\tau_{4}$ is fixed by either $D$-terms or $g_{s}$ corrections at the quiver locus or in the geometric regime, the supergravity $F$-term scalar potential reads:

$$
V=\frac{8 a_{3}^{2} A_{3}^{2}}{3 \alpha \gamma_{3}}\left(\frac{\sqrt{\tau_{3}}}{\mathcal{V}}\right) e^{-2 a_{3} \tau_{3}}-4 W_{0} a_{3} A_{3}\left(\frac{\tau_{3}}{\mathcal{V}^{2}}\right) e^{-a_{3} \tau_{3}}+\frac{3 \xi W_{0}^{2}}{4 g_{s}^{3 / 2} \mathcal{V}^{3}}
$$

This potential completely stabilises $\tau_{3}$ and the volume $\mathcal{V} \simeq \alpha \sqrt{\tau_{1}} \tau_{2}$ at:

$$
\left\langle\tau_{3}\right\rangle=\frac{1}{g_{s}}\left(\frac{\xi}{2 \alpha \gamma_{3}}\right)^{\frac{2}{3}}, \quad\langle\mathcal{V}\rangle=\left(\frac{3 \alpha \gamma_{3}}{4 a_{3} A_{3}}\right) W_{0} \sqrt{\left\langle\tau_{3}\right\rangle} e^{a_{3}\left\langle\tau_{3}\right\rangle} .
$$

The direction in the $\left(\tau_{1}, \tau_{2}\right)$-plane orthogonal to the overall volume is still flat. It can be stabilised by the inclusion of string loop corrections to the Kähler potential which arise once we wrap a stack of $D 7$ branes around $\tau_{1}$ and another stack around $\tau_{2}$. These two stacks can correspond to either a hidden or a visible sector. These $g_{s}$-corrections turn out to be subleading with respect to (2.24), due to the extended no-scale structure [13, and so they do not destroy the large volume minimum (2.25) but they can lift the remaining flat direction. The loop generated potential looks like [21]:

$$
\delta V_{\left(g_{s}\right)}=\left(\frac{A}{\tau_{1}^{2}}-\frac{B}{\mathcal{V} \sqrt{\tau_{1}}}+\frac{C \tau_{1}}{\mathcal{V}^{2}}\right) \frac{W_{0}^{2}}{\mathcal{V}^{2}}
$$

and this fixes $\tau_{1}$ at:

$$
\left\langle\tau_{1}\right\rangle=c \mathcal{V}^{2 / 3}, \quad \text { where } \quad c \simeq \frac{\left(g_{s} \mathcal{C}_{1}\right)^{4 / 3}}{\mathcal{C}_{12}^{2 / 3}} .
$$

As shown in appendix A, the direction $\chi$ fixed by the string loops, turns out to be naturally lighter than the volume mode which sets the scale of the potential: $V \sim M_{P}^{2} m_{\mathcal{V}}^{2}$. Therefore this field is a natural candidate to drive inflation given that the $\eta$-parameter looks like:

$$
\eta=M_{P}^{2} \frac{V_{\chi \chi}}{V} \simeq \frac{m_{\chi}^{2}}{m_{\mathcal{V}}^{2}} \simeq \frac{\mathcal{V}^{-10 / 3}}{\mathcal{V}^{-3}} \simeq \frac{1}{\mathcal{V}^{1 / 3}} \ll 1
$$

Now working in the $\left(\mathcal{V}, \tau_{1}\right)$-plane and displacing $\tau_{1}$ far from its minimum, due to the fact that this mode is naturally lighter than $H$ by a factor $\mathcal{V}^{-1 / 3} \ll 1$, the K3 fibre experiences a very flat direction which is suitable for inflation. In terms of the canonically normalised inflaton $\phi=(\sqrt{3} / 2) \ln \tau_{1}$, the inflationary potential looks like 21]:

$$
V=\frac{\beta}{\mathcal{V}^{10 / 3}}\left(3-4 e^{-\phi / \sqrt{3}}+e^{-4 \phi / \sqrt{3}}+R e^{2 \phi / \sqrt{3}}\right),
$$

\footnotetext{
${ }^{4}$ Even in this case, in order not to have $\left\langle\tau_{3}\right\rangle \ll 1, \tau_{3}$ has to be wrapped by a stack of $D 7$-branes supporting a hidden sector where gaugino condensation takes place.
} 
where

$$
R \simeq g_{s}^{4} \ll 1 .
$$

The potential in the inflationary region is dominated by the up-lifting bit plus the first negative exponential in (2.29), and so it can be very well be approximated as:

$$
V \simeq \frac{\beta}{\mathcal{V}^{10 / 3}}\left(3-4 e^{-\phi / \sqrt{3}}\right)
$$

which is a typical large-field inflaton potential that can give rise to observable gravity waves due to a trans-Planckian motion of $\phi$ in field space: $\Delta \phi \gtrsim M_{P}$. It is also interesting to notice that all the adjustable parameters enter only in the prefactor rendering this inflationary model very predictive. The slow-roll parameters $\epsilon$ and $\eta$ are naturally smaller than unity for large $\phi$. In addition both the tensor-to-scalar ratio $r$ and the spectral index $n_{s}$ are functions of just the number of e-foldings $N_{e}$, and they can be expressed one in terms of the other via the interesting relation 21]:

$$
r \simeq 6\left(n_{s}-1\right)^{2} .
$$

The requirement of generating enough density perturbations fixes $\mathcal{V} \simeq 10^{3-4}$, corresponding to a GUT-scale $M_{s}$. Then for different values of the reheating temperature $T_{R H}$, one can obtain a different number of e-foldings $N_{e}$, which, in turn, fixes the predictions for the cosmological observables $r$ and $n_{s}$. This prediction does not depend strongly on $T_{R H}$, and one generically obtains the promising outcome [21]:

$$
n_{s}=0.97, \quad \text { and } r=0.005,
$$

with a tensor-to-scalar ratio potentially detectable by future cosmological observations.

Let us make a few final remarks:

- As in the case of BI, also in FI, both in the geometric regime and at the quiver locus, it is impossible to build a model without the presence of a hidden sector. In fact, in FI a stack of $D 7$-branes wrapping the two large cycles $\tau_{1}$ and $\tau_{2}$ has to be present in order to generate the string loop corrections that fix the K3 fibre and provide the inflationary potential. In addition the blow-up cycle $\tau_{3}$ has still to support a hidden sector that undergoes gaugino condensation in order to stabilise the overall volume at a phenomenologically viable value.

- In section 2.2.1 we noticed that $g_{s}$ corrections in general would destroy BI, whereas for FI we have seen that the inflationary potential is loop-generated. So in one case, string loops destroy inflation whereas in the other case they give rise to a nice potential naturally suitable to drive inflation. The reason of this different behaviour of the same kind of corrections is the different topology of the inflaton 4-cycle, which in the first case is the size of a blow-up mode resolving a point-like singularity whereas in the second case, it is the volume of a K3-fibre. The different topology is reflected in a different volume-scaling of the elements of the inverse Kähler metric which enter into the $F$-term supergravity scalar potential.

- FI requires a rather high string-scale which would seem to be in disagreement with its intermediate value that was needed to obtain TeV scale SUSY for the models in the geometric regime. Hence this would seem to be another indication in favour of LVS at the quiver locus. However it has to be said that the potential of FI is able to give inflation for all scales and the only issue which sets $M_{s} \sim M_{G U T}$, is the requirement of generating enough density perturbations. Therefore one could try to let the inflaton just drive inflation and generate the density fluctuations via another curvaton-like or modulating field. In this way, it might be possible either to lower the inflationary scale or to generate large non-Gaussianities in the CMB spectrum [23], but one should worry about excess isocurvature perturbations as the inflaton and curvaton decay products might not thermalise before BBN 2 . 


\subsection{Moduli dynamics after inflation and reheating}

At the end of inflation, the inflaton field which acts like a homogeneous condensate oscillates coherently around its minimum. During this time there could be perturbative and non-perturbative ways of creating particles which are coupled to the inflaton [2, 3]. There could be various distinct ways of exciting non-perturbative particles in general:

1. Tachyonic preheating: if there exists a scalar potential where the effective mass of the inflaton becomes $m_{\text {eff }}^{2}<0$, then there arises an instability [37] for the modes $k \leq m_{e f f}$. The occupation number of the inflaton quanta grows exponentially, $n_{k} \sim \exp \left(2 m_{\text {eff }} t_{*}\right)$, where $t_{*}$ is a typical time scale during which the growth of the momentum mode $k$ takes place. For a typical self coupling of the inflaton, $\lambda$, the growth factor can be at most $n_{k} \sim \pi^{2} / \lambda$. Note that for smaller $\lambda$ the occupation number can be larger. However this alone does not drain the inflaton energy density into visible or hidden dof since this process simply excites the self quanta of the inflaton [37]. The inflaton still needs to decay perturbatively into the observable and hidden dof in order to reach full thermalisation.

2. Instant reheating: if the inflaton couples to visible sector dof, it is possible to excite them just within few oscillations. This happens typically for very large Yukawa or gauge couplings in a renormalisable interaction. Note that by virtue of the inflaton coupling to visible sector dof, they obtain time dependent masses during the inflaton oscillations. However this mass vanishes if the inflaton passes through a zero VEV. It is then more effective to excite the zero modes of the visible sector dof from the vacuum fluctuations. In a typical scenario, a zero VEV would correspond to a point of enhanced gauge symmetry, where the gauge bosons are effectively massless [5. Particle production happens due to the violation of the adiabaticity condition: $\dot{\omega}_{k} \gg \omega_{k}^{2}$, where $\omega_{k}$ is the frequency of the excited quanta with momentum $k 38$, 39]. However in our case the instant preheating is rather unlikely, as the inflaton couplings to hidden and visible sectors are governed by non-renormalisable interactions. Since the couplings are so weak, the excitations of the visible sector dof do not exhaust the inflaton energy density.

3. Parametric resonance: in this case the particle creation mechanism is similar to the instant reheating scenario, albeit particle creation takes place in each oscillation whenever the inflaton passes through its minimum. If the inflaton couples to ordinary matter and gauge fields, it can then excite these dof from the time dependent induced mass term [39, 40]. In both instant and parametric resonance the particle creation mechanism is based on the violation of the adiabaticity condition. In the later case particle creation happens continuously over the oscillations. In both cases the visible sector dof can be excited if the couplings are large. However the decay products have a non-thermal spectra and a complete thermalisation of all the dof happens at a much larger time scale which is comparable to that of the perturbative decay of the inflaton, i.e. $\Gamma_{\text {infl }}^{-1}$ (see [3, 41]). Furthermore notice that in order to excite all the MSSM and hidden dof the inflaton has to decay completely, which happens only perturbatively.

4. Fragmentation: if the inflaton possesses a global or gauged $U(1)$ symmetry then it is possible to fragment the inflaton condensate to form inflatonic Q-balls [6, 42] provided the effective potential near the minimum grows slower than $\phi^{2}$ by virtue of quantum corrections. These inflatonic non-topological solitons, known as Q-balls, decay into lighter fermions from their surface and in some cases can be absolutely stable if the energy of the Q-ball becomes smaller than the lightest baryons in the theory [6]. The stable Q-balls can become a dark matter component of the Universe [43]. 
5. Reheating and thermalisation: given that all the above mentioned effects are typically scatterings of the inflaton to excite new quanta, preheating cannot exhaust all the inflaton energy density. Preheating will end when the backreaction of the excited quanta becomes important. The inflaton then must decay perturbatively. The decay products of the inflaton and the excited quanta due to preheating must thermalise to produce a thermal bath of MSSM dof. Typically thermalisation of all the dof take considerably longer time than one would expect, especially in the context of the MSSM [44, 45, 46. One of the main reasons is that the MSSM contains $F$-and $D$-flat directions (parametrised by a complex scalar monomial field), which are made up of MSSM squarks and sleptons [6]. During inflation these flat directions tend to take large VEVs by virtue of random quantum fluctuations imparted on light scalars with $\mathrm{TeV}$ scale mass due to a large Hubble friction term. Once these flat directions take a large VEV during inflation, their VEV slowly rolls down towards their minimum. Since these flat directions are charged under the MSSM gauge group, during their dynamical evolution after inflation they produce VEV dependent masses to gauge bosons and gauginos similar to the Higgs mechanism. Heavy gauge bosons and gauginos tend to slow down any $2 \leftrightarrow 2$ or $2 \leftrightarrow 3$ interactions required to obtain kinetic and chemical equilibrium, which are essential ingredients for acquiring local thermodynamical equilibrium [44]. These flat directions have conserved global numbers, like baryonic, leptonic or some combination of both, which enable them to live long enough and decay perturbatively close to their $\mathrm{TeV}$ mass scale 46. One of the important consequences of the finite VEV of the flat direction is that they can kinematically block preheating all together [3, 45].

The bottom line is that the thermalisation time scale $\Gamma_{\text {therm }}^{-1}$ in the presence of MSSM flat directions in the visible sector can be longer than the time scale of the perturbative decay of the inflaton, $\Gamma_{\text {infl }}^{-1}: \Gamma_{\text {infl }}^{-1} \leq \Gamma_{\text {therm }}^{-1} \simeq H_{\text {therm }}^{-1}$. The final reheating temperature will then be given by [44]:

$$
T_{R H}^{\text {therm }} \simeq\left(\Gamma_{\text {therm }} M_{P}\right)^{1 / 2} .
$$

Since $\Gamma_{\text {therm }}$ depends on the nature of the MSSM flat directions and their dynamics, we will leave this detailed study for future investigation. In a rather model independent scenario, it is expected that a complete thermalisation will take place when the flat directions completely evaporate. This happens at temperatures fairly close to the TeV scale [44, 45, 46].

For the purpose of illustrating the current issues at hand we shall work under the approximation of sudden thermalisation of the decayed particles, which are assumed to be relativistic at the time of decay. Thus we shall obtain a maximal value for the reheating temperature which we shall denote as $T_{R H}^{\max }$. This implicitly assumes also that the perturbative decay channels are the main source of energy transfer from the moduli sector. Given the couplings of the moduli to hidden and visible sectors to be so weak, preheating effects are expected to be largely suppressed. By equating the inflaton decay rate to the Hubble parameter for radiation dominance by assuming $\Gamma_{i n f l} \simeq H$, we obtain:

$$
T_{R H}^{\text {therm }} \leq T_{R H}^{\max } \simeq\left(\Gamma_{\text {infl }} M_{P}\right)^{1 / 2} .
$$

Note that since in our case the visible and hidden sectors do not have any direct coupling, the two sectors are not expected to thermalise before BBN. For all practical purposes the hidden sector will become like a dark sector. Thus there will be two separate thermal baths formed with two distinct temperatures, $T_{v i s}$ and $T_{h i d}$ both of them of the order $T_{R H}^{\max }$, and $d o f, g_{*}^{v i s}$ and $g_{*}^{\text {hid }}$, which we assume to be relativistic:

$$
H^{2} \simeq g_{*}^{v i s} \frac{T_{v i s}^{4}}{M_{P}^{2}}+g_{*}^{h i d} \frac{T_{h i d}^{4}}{M_{P}^{2}} .
$$


In this paper we will do the first preliminary check regarding the inflaton decay rates to visible and hidden dof. It is then important to know what fraction of the inflaton energy density gets diverted into the hidden sector. Since in our case a Kähler modulus plays the rôle of the inflaton within the 4D effective field theory, it is crucial to derive the inflaton couplings to visible and hidden sector dof which are localised in particular regions of the Calabi-Yau compactification. This derivation is performed in detail in appendix A. We also stress that in sections 2.2.1 and 2.3 we showed that it is impossible to avoid the presence of hidden sectors. Therefore a full understanding of reheating requires a non-trivial interplay between local and global aspects of string compactifications but it can also set severe constraints on which internal 4-cycle can be wrapped by what kind of branes.

There could be three potential problems which may arise in the study of reheating:

- The inflaton decay rate to visible sector dof is suppressed with respect to its decay rate to hidden sector dof by some power of the overall volume:

$$
\frac{\Gamma_{\text {infl } l \rightarrow \text { vis.sec. }}}{\Gamma_{\text {infl } l \rightarrow \text { hid.sec. }}} \sim \mathcal{O}\left(\frac{1}{\mathcal{V}^{p}}\right) \ll 1, \text { for } p>0 .
$$

In this case the inflaton dumps all its energy into hidden sector, instead of visible sector, dof, and so it is impossible to reheat the visible sector. Notice that the reheating of the visible sector cannot proceed via the decay of the hidden sector particles to the visible ones. In fact there is no direct coupling between these particles since they correspond to two open string sectors localised in different regions of the Calabi-Yau. The only indirect gravitational coupling via a graviton or a modulus exchange is definitely too weak to give rise to a viable reheating of the visible sector due to the decay of hidden to visible $d o f$.

- The inflaton decay rate to visible and hidden sector dof has the same volume scaling:

$$
\frac{\Gamma_{\text {infl } l \rightarrow \text { vis.sec. }}}{\Gamma_{\text {infl } l \rightarrow \text { hid.sec. }}} \sim \mathcal{O}(1) .
$$

In this case the inflaton decays at the same time to hidden and visible sector particles releasing the same amount of entropy to both sectors. This might generically yield an overproduction of dark matter particles given by the stable hidden superpartners which interact with the visible dof only gravitationally and get an $\mathcal{O}\left(M_{\text {soft }}\right)$ mass due to SUSY-breaking effects. These dark matter particles can be produced either non-thermally directly by the inflaton decay or by thermal effects. In the last case, two different thermal baths get formed with temperatures $T_{v i s}$ and $T_{h i d}$, with $T_{v i s} \neq T_{h i d}$, and the hidden sector dark matter particles would be created thermally if $T_{\text {hid }}>M_{\text {soft }}$.

- The inflaton decay rate to hidden sector dof is subleading with respect to its decay rate to visible sector $d o f$ :

$$
\frac{\Gamma_{\text {infl } l \rightarrow \text { hid.sec. }}}{\Gamma_{\text {infl } \rightarrow \text { vis.sec. }}} \sim \mathcal{O}\left(\frac{1}{\mathcal{V}^{p}}\right) \ll 1, \text { for } p>0 .
$$

In this case the inflaton can reheat the visible sector but the reheating temperature can turn out to be lower than the BBN temperature $T_{B B N} \sim 1 \mathrm{MeV}: T_{R H}^{\max }<T_{B B N}$, due to an effective decoupling of the inflaton from the visible sector because of the geometrical separation of the two corresponding 4-cycles within the Calabi-Yau.

\section{Hidden sector dynamics}

As we have seen in the previous section, in order not to have an overall volume which is too large for VEVs of the moduli larger than the string scale, one is forced to consider non-perturbative 
effects coming from gaugino condensation. Thus we need a hidden stack of $D 7$-branes supporting a supersymmetric field theory that undergoes gaugino condensation.

Let us focus on a $4 \mathrm{D} N=1 S U\left(N_{c}\right)$ gauge theory with $N_{f}$ chiral superfields in the fundamental representation, $Q_{a}^{i}$, with $a=1, \ldots, N_{c}$ and $i=1, \ldots, N_{f}$, and $N_{f}$ chiral superfields in the antifundamental representation, $\bar{Q}_{i}^{a}$ (for a review see [47]). The global anomaly-free symmetry is $S U\left(N_{f}\right)_{L} \times S U\left(N_{f}\right)_{R} \times U(1)_{B} \times U(1)_{R}$. The unique gauge-invariant chiral superfield is $M_{i j}=Q_{i} \cdot \bar{Q}_{j}$ and it can be thought of as a meson superfield whose scalar component is a colour-singlet combination of squarks. This theory gives rise to gaugino condensation for $N_{f}<\left(N_{c}-1\right)$, and so we shall focus only on this case ${ }^{5}$. Let us analyse the two subcases $N_{f}=0$ and $N_{f} \neq 0$ separately.

\section{$3.1 N_{f}=0$}

For $N_{f}=0$, we are in the case of a pure supersymmetric Yang-Mills theory with no matter. Defining the fundamental coupling constant $g^{2}$ at the UV scale $\Lambda_{U V}$, the effective running coupling constant is given by:

$$
\frac{1}{g^{2}}(\mu)=\frac{1}{g^{2}}+\frac{\beta}{16 \pi^{2}} \ln \left(\frac{\Lambda_{U V}^{2}}{\mu^{2}}\right) .
$$

This theory confines in the IR due to strong dynamics at the scale $\Lambda$ where the expression (3.1) formally diverges:

$$
\Lambda^{\beta}=\Lambda_{U V}^{\beta} e^{-8 \pi^{2} / g^{2}}
$$

where the coefficient of the 1-loop $\beta$-function is given by $\beta=3 N_{c}$. In addition, at the scale $\Lambda$ the theory develops a mass gap since glueballs $(g g),\left\langle F_{\mu \nu} F^{\mu \nu}\right\rangle \sim \Lambda^{4}$, gluinoball-mesons $(\tilde{g} \tilde{g}),\langle\lambda \lambda\rangle \sim \Lambda^{3}$ and 'glueballinos' $(g \tilde{g})$, all get a mass of the order $\Lambda$. The resulting non-perturbative superpotential looks like:

$$
W_{n p}=\Lambda^{3}=\Lambda_{U V}^{3} e^{-8 \pi^{2} /\left(N_{c} g^{2}\right)} .
$$

Notice that these condensates break the original $\mathbb{Z}_{2 N}$ global symmetry but since this is a discrete symmetry no massless Goldstone boson arises.

In string compactifications, the fundamental gauge coupling is given by the modulus $\tau$ supporting this pure $N=1$ SYM theory, $g^{-2}=\tau /(4 \pi)$, and so we can integrate out all the condensates and remain with a holomorphic non-perturbative superpotential for $\tau=\operatorname{Re}(T)$ below the scale $\Lambda$ :

$$
W_{n p}=M_{P}^{3} e^{-2 \pi T / N_{c}} .
$$

Let us estimate the order of magnitude of $\Lambda$. There are two ways to do it: either from (3.3) or from the expression of the normalised superpotential $e^{K / 2} W_{n p}=\Lambda^{3}$ in terms of (3.4). From (3.3) we obtain:

$$
\Lambda=\Lambda_{U V} e^{-a \tau / 3}, \quad \text { with } \quad a \equiv 2 \pi / N_{c} .
$$

There is a subtlety now pointed out in [33]. It might be that in the case when the cycle is invariant under the orientifold then the running of the coupling starts from $M_{s}$ while when the cycle is not invariant under the orientifold the UV scale is higher: $\Lambda_{U V}=M_{s} R \simeq M_{s} \mathcal{V}^{1 / 6}$. In the first case we would obtain:

$$
\Lambda \simeq \frac{M_{P}}{\mathcal{V}^{1 / 2}} e^{-a \tau / 3}
$$

while in the second case we find:

$$
\Lambda \simeq \frac{M_{P}}{\mathcal{V}^{1 / 3}} e^{-a \tau / 3} .
$$

\footnotetext{
${ }^{5}$ For $N_{f}=\left(N_{c}-1\right)$, the non-perturbative superpotential is generated by gauge instantons and so we are not interested in this case. In addition no non-perturbative superpotential is generated for $N_{f}>\left(N_{c}-1\right)$.
} 
On the other hand, from (3.4) we realise that:

$$
\Lambda=e^{K / 6} W_{n p}^{1 / 3}=e^{K / 6} M_{P} e^{-a \operatorname{Re}(T) / 3} \simeq \frac{M_{P}}{\mathcal{V}^{1 / 3}} e^{-a \operatorname{Re}(T) / 3} .
$$

Comparing (3.8) with (3.7), we see that in the second case we have $\operatorname{Re}(T)=\tau$, but comparing (3.8) with (3.6) we realise that in the first case we need to redefine the modulus as $\operatorname{Re}(T)=\tau+\ln \left(\mathcal{V}^{1 / 2}\right) / a$ [33]. Given that in both cases $e^{-a \tau} \simeq \mathcal{V}^{-1}$, we end up with $\Lambda \simeq M_{P} \mathcal{V}^{-5 / 6}$ for the first case, and $\Lambda \simeq M_{P} \mathcal{V}^{-2 / 3}$ for the second.

The scale of IR strong dynamics $\Lambda$ has to be no lower than the Hubble parameter during inflation $H_{\text {inf }}: H_{\text {inf }} \leq \Lambda$ [36. This is indeed the case for FI:

$$
H_{i n f} \simeq \frac{V^{1 / 2}}{M_{P}} \simeq \frac{M_{P}}{\mathcal{V}^{5 / 3}}
$$

and for BI when $\tau$ sits at its VEV:

$$
H_{i n f} \simeq \frac{V^{1 / 2}}{M_{P}} \simeq \frac{M_{P}}{\mathcal{V}^{3 / 2}} .
$$

However, as we pointed out in section 2.2.1, the non-perturbative inflationary potential for BI has to be generated by gaugino condensation and not by stringy instantons. Therefore in this case, given that the inflaton $\tau$ varies during inflation, also $\Lambda$ varies while $H_{\text {inf }}$ remains constant. Thus we need to check that $H_{\text {inf }}<\Lambda$ throughout all inflationary dynamics and not just when $\tau$ relaxes at its minimum. Focusing on the case when the 4-cycle is invariant under the orientifold, we have to make sure that the expression (3.6) for $\Lambda$ evaluated at the value of $\tau$ such that you get 60 e-foldings of inflation, is not lower than $H_{\text {inf }}$ given by (3.10). As can be seen from Table 1, taking $a \tau=n \ln \mathcal{V}$, small variations of $n$ give large modifications of the number of e-foldings. In particular, $N_{e}=60$ is obtained for $n \simeq 2.7$ if $\mathcal{V} \simeq 10^{6}$. Then we obtain:

$$
\Lambda \simeq \frac{M_{P}}{\mathcal{V}^{1 / 2}} e^{-a \tau / 3} \simeq \frac{M_{P}}{\mathcal{V}^{1 / 2}} e^{-(\ln \mathcal{V})^{9 / 10}} \simeq \frac{M_{P}}{\mathcal{V}^{7 / 5}} \Rightarrow \frac{H_{\text {inf }}}{\Lambda} \simeq \frac{1}{\mathcal{V}^{1 / 10}} \leq \mathcal{O}(1),
$$

and so we conclude that throughout all inflationary dynamics we always have $H_{\text {inf }} \leq \Lambda$.

Another very interesting observation is that the inflaton mass $m_{\text {inf }}$ after inflation for both BI and FI is lower than $\Lambda$ since we have:

$$
m_{\text {inf }} \simeq \frac{M_{P}}{\mathcal{V}} \text { for BI, } \quad m_{\text {inf }} \simeq \frac{M_{P}}{\mathcal{V}^{5 / 3}} \text { for FI. }
$$

Thus the inflaton decay to hidden sector dof is kinematically forbidden! In addition the KaluzaKlein scale is given by:

$$
M_{K K} \simeq \frac{M_{s}}{R} \simeq \frac{M_{s}}{\tau_{4}^{1 / 4}} \simeq \frac{M_{P}}{\sqrt{\mathcal{V}} \tau_{4}^{1 / 4}},
$$

and so we end up with the following final hierarchy among scales:

$$
m_{\text {inf }}<H_{\text {inf }} \leq \Lambda<M_{K K}<M_{s} .
$$

Finally we need also to check that the gaugino condensate does not get destabilised by thermal effects as it would be the case for reheating temperatures in the hidden sector $T_{\text {hid }}>\Lambda$. However we can check that this is never the case. The largest temperature in the hidden sector is given by $T_{\text {hid }} \simeq\left(\Gamma_{\text {inf }} M_{P}\right)^{1 / 2}$, where $\Gamma_{\text {inf }}=m_{\text {inf }}^{3} \mathcal{V}^{x} / M_{P}^{2}$ is the inflaton decay rate. Given that the strongest possible inflaton coupling is $1 / M_{s}$ we infer that $x \leq 1$, and so it suffices to check that $T_{\text {hid }}<\Lambda$ for $x=1$. However in this case we can conclude that we always have $T_{h i d}<\Lambda$ since:

$$
\frac{T_{h i d}}{\Lambda} \simeq \frac{1}{\mathcal{V}^{1 / 6}}<1 \text { for BI, } \quad \frac{T_{h i d}}{\Lambda} \simeq \frac{1}{\mathcal{V}^{7 / 6}}<1 \text { for FI. }
$$


Let us see now how an $N=1 S U\left(N_{c}\right)$ gauge theory with $N_{f}=0$ can be realised in type IIB string compactifications. All the cycles which in our scenarios are stabilised non-perturbatively are local blow-up modes, and so they are rigid cycles which do not intersect with any other cycle. Thus these cycles are perfect to generate no matter either from possible intersections with other cycles or from deformations of the cycle. Given that these cycles are non-Spin, one has to turn on half-integer world-volume fluxes to cancel the Freed-Witten anomalies [48. These fluxes could generate chiral matter but since these cycles do not intersect with any other cycle, one can always choose the $B$-field to compensate these Freed-Witten fluxes and have a total world-volume flux which is zero ${ }^{6}$.

Let us see now what happens if one wraps a stack of $D 7$-branes around a local blow-up 4-cycle which is invariant under the orientifold so that the $D 7$-branes are on top of the $O 7$-planes ${ }^{7}$. In order to cancel the tadpole, $4 \mathrm{D} 7$-branes have to sit on top of each $O 7$-plane. The gauge theory on each stack of $N D$ 7-branes is $S O(2 N)$ and so the gauge group is $S O(8)^{n}$ for $n O 7$-planes. In addition one can turn on a world-volume Abelian gauge flux $F_{2}$ on this 4-cycle: $F_{2}=T_{0} F_{2}^{(0)}+T_{i} F_{2}^{(i)}$, where $T_{0}$ is the generator of the diagonal $U(1)_{d} \subset S O(2 N)$ while $T_{i}$ are the traceless Abelian elements of $S U(N)$. It is then important to consider what happens to $F_{2}$ :

1. $F_{2}=0$ and so we obtain a pure $N=1 S O(8)^{n}$ gauge theory that undergoes gaugino condensation and develops a mass-gap.

2. Only a diagonal flux is turned on: $F_{2}=T_{0} F_{2}^{(0)}$. This flux breaks the gauge group $S O(2 N) \rightarrow$ $S U(N) \times U(1)_{d}$ and the $U(1)_{d}$ acquires a string-scale mass via the Green-Schwarz mechanism. No chiral matter is generated and the theory below $M_{s}$ is still $N=1$ pure SYM. However the non-zero gauge flux generates a Fayet-Iliopoulos term $\xi$ associated to $U(1)_{d}$ which cannot be cancelled by any scalar field. Hence in order to cancel the $D$-terms, one has to impose $\xi=0$ which, due to the $\tau$-dependence of $\xi$, implies that the cycle supporting the hidden sector is forced to shrink to zero size 15 . This is what we do not want to have both for the inflaton and for the cycle keeping the volume minimum stable during inflation, and so we need to discard this case.

3. Only some of the $D 7$-branes get magnetised, say just one of them: $F_{2}=T_{0} F_{2}^{(0)}+T_{1} F_{2}^{(1)}$. This flux breaks the gauge group $S O(2 N) \rightarrow S U(N) \times U(1)_{d} \rightarrow S U(N-1) \times U(1)_{1} \times U(1)_{d}$. The new $U(1)_{1}$ gets massive via the Green-Schwarz mechanism only if $F_{2}^{(1)}$ is turned on on a 2-cycle which is also a 2-cycle of the Calabi-Yau. In addition, chiral matter in the fundamental representation gets originated and so one has to check that $N_{f}<\left(N_{c}-1\right)$ in order to have still gaugino condensation.

Notice that in this case one can prevent the cycle to shrink to zero size by cancelling the Fayet-Iliopoulos term against the VEV of a scalar field $\Phi$ charged under the anomalous $U(1)$ which becomes massive: $\langle\Phi\rangle \sim \sqrt{\xi} \sim M_{s}$. If then the superpotential contains a term of the form:

$$
W=\lambda \Phi Q_{i} \cdot \bar{Q}_{j},
$$

${ }^{6}$ Let us illustrate this claim with a simple example. Let $\Sigma_{1}$ and $\Sigma_{2}$ be two blow-up 4-cycles which do not intersect with each other, and let $D_{1}$ and $D_{2}$ be the corresponding dual 2-forms. Then the half-integer Freed-Witten fluxes $F_{1}$ and $F_{2}$ will look like:

$$
F_{1}=\frac{f_{1}}{2} \cdot D_{1}+0 \cdot D_{2} \quad \text { and } \quad F_{2}=0 \cdot D_{1}+\frac{f_{2}}{2} \cdot D_{2},
$$

with $f_{1}$ and $f_{2}$ integer coefficients. The $B$-field can be adjusted to take the form $B=F_{1}+F_{2}$, so that the total world-volume flux $\left(B-F_{i}\right)$ on $\Sigma_{i}, i=1,2$, is vanishing. Notice that if the two cycles intersected, then $B$ would not be able to compensate both half-integer fluxes at the same time.

${ }^{7}$ We shall not consider the case when the cycle is not invariant under the orientifold due to the similarity with instantons which contribute to the superpotential only if the cycle wrapped by the instanton is invariant under the orientifold, and the fact that it would be difficult to satisfy tadpole cancelation. 
the VEV of $\Phi$ generates a mass for the matter fields of the order $M_{s}>\Lambda$ (for $\lambda \sim 1$ ). Therefore the matter fields can be safely integrated out obtaining a pure $N=1$ SYM theory that develops a mass gap [49].

$3.2 N_{f}<\left(N_{c}-1\right)$

For $N_{f}<\left(N_{c}-1\right)$, the original $S U\left(N_{c}\right)$ gauge theory has $2 N_{f} N_{c}$ complex scalars. Then $D$-terms break the gauge group to $S U\left(N_{c}-N_{f}\right)$ and the resulting theory consists of $\left(N_{c}-N_{f}\right)^{2}-1$ gauge bosons and gauginos plus $N_{f}^{2}$ scalar moduli $M_{i j}$ 47]. The non-perturbative superpotential for $M_{i j}$ is generated by gaugino condensation. This is the famous Affleck-Dine-Seiberg superpotential whose form can be constrained using symmetries [50]:

$$
W_{n p} \simeq\left(\frac{\Lambda^{3 N_{c}-N_{f}}}{\operatorname{det}(M)}\right)^{\frac{1}{N_{c}-N_{f}}} .
$$

Notice that for $N_{f}=0$ we get the previous expression (3.3): $W_{n p} \simeq \Lambda^{3}$. The states $g g, \lambda \lambda$ and $g \lambda$ get a mass of the order $\Lambda$ but the superpotential (3.18) gives a run-away for $M_{i j}$. Therefore there is no stable minimum and the system is driven to $\left\langle M_{i j}\right\rangle \rightarrow \infty$. Notice that the $F$-term of $M_{i j}$ gives the quark condensate: $F_{M_{i j}} \propto\left\langle\psi_{Q_{i}} \cdot \psi_{\bar{Q}_{j}}\right\rangle$. In addition $F_{M_{i j}} \sim\left\langle M_{i j}\right\rangle^{p}$ with $p<0$, hence $\left\langle M_{i j}\right\rangle \rightarrow \infty$ implies $\left\langle\psi_{Q_{i}} \cdot \psi_{\bar{Q}_{j}}\right\rangle \rightarrow 0$. Thus the theory does not show any spontaneous breaking of the original chiral symmetry $S U\left(N_{f}\right)_{L} \times S U\left(N_{f}\right)_{R}$.

However now we are working in supergravity where, due to the $e^{K}$ prefactor of the scalar potential, $M_{i j}$ can be stabilised at a finite VEV. In addition in flux compactifications the background fluxes break supersymmetry inducing a non-zero $F$-term for the modulus supporting the hidden sector $^{8}$. This generates a SUSY-breaking mass term for the scalars: $\Delta \mathcal{L}=-m_{0}^{2}\left(|Q|^{2}+|\bar{Q}|^{2}\right)$, that is the main contribution that yields a minimum at finite $\left\langle M_{i j}\right\rangle=t_{\star} \delta_{i j}$, with $t_{\star} \sim\left(\frac{\Lambda^{k_{1}}}{m_{0}}\right)^{k_{2}}$, $k_{1}=\frac{5 N_{c}-3 N_{f}}{N_{c}-N_{f}}$ and $k_{2}=\frac{N_{c}-N_{f}}{2 N_{c}-N_{f}}$ 51. Expanding around this VEV all the fermions get a mass of the order $m_{0}$. Also some scalars get a mass of the order $m_{0}$ but some of them are left massless. These are the Goldstone bosons of the spontaneously broken chiral symmetry due to the presence of mass terms: $S U\left(N_{f}\right)_{L} \times S U\left(N_{f}\right)_{R} \rightarrow S U\left(N_{f}\right)_{V}$. Notice that now $\left\langle\psi_{Q_{i}} \cdot \psi_{\bar{Q}_{j}}\right\rangle \neq 0$.

This discussion and the previous one for the case $N_{f}=0$ suggest that an $N=1 S U\left(N_{c}\right)$ gauge theory with $N_{f} \neq 0$ can be realised in type IIB string compactifications in several ways. Focusing on local blow-up modes invariant under the orientifold, one has to magnetise only some of the $D 7$ branes wrapping this cycle checking that $N_{f}$ chiral flavours are generated with $N_{f}<\left(N_{c}-1\right)$. Then one has to make sure that the cycle does not shrink to zero size by cancelling the Fayet-Iliopoulos term associated to anomalous $U(1)$ factors against the VEV of a scalar, but without generating a mass term of the order $M_{s}$ for the matter fields due to couplings like (3.17). Then the theory undergoes gaugino condensation, and in the presence of chiral symmetry, its spontaneous breaking will always give rise to massless Goldstone bosons except for the case of a low energy Higgs-like mechanism.

\subsection{Constraint on hidden sector configurations}

We have seen that the simple requirement of having a hidden sector that undergoes gaugino condensation supported by a rigid orientifold-invariant 4-cycle which does not shrink at the quiver locus and does not intersect with any other cycle, does not restrict the possible particle content and mass spectrum of the hidden sector. Let us summarise the various options which we have found:

\footnotetext{
${ }^{8}$ Notice that there are constructions where gaugino condensation can restore supersymmetry but this is never the case for LVS.
} 
1. The hidden sector consists just of a pure $N=1$ SYM theory that develops a mass-gap. All particles acquire a mass of the order $\Lambda$ and are heavier than the inflaton.

2. The hidden sector consists of a pure $N=1$ SYM theory that develops a mass-gap plus a massless $U(1)$. The mass-spectrum below $\Lambda$ consists of massless hidden photons and hidden photini which get an $\mathcal{O}\left(M_{\text {soft }}\right)$ mass due to SUSY-breaking effects.

3. The hidden sector consists of an $N=1 S U\left(N_{c}\right)$ theory with $N_{f}<\left(N_{c}-1\right)$ flavours. Furthermore there might be an additional massless $U(1)$. The condensates made of gauge bosons and gauginos get a mass of the order $\Lambda$. In the presence of spontaneous chiral symmetry breaking some matter condensates are massless while the other get a mass of the order $M_{\text {soft }}$. If chiral symmetry is explicitly broken by a low energy Higgs-like mechanism, all the matter fields will get a $\delta m \ll M_{\text {soft }}$ contribution to their masses.

Therefore there are two options for the matter spectrum:

(a) The hidden photons are massless while the hidden photini, matter fermionic and scalar condensates acquire a mass of the order $M_{\text {soft }}$. In addition there are very light (or even massless) pion-like mesons.

(b) There are matter fermionic and scalar condensates with mass of the order $M_{\text {soft }}$ and very light (or even massless) pion-like mesons, but all the hidden gauge bosons and gauginos are heavier than the inflaton.

\section{Inflaton couplings to hidden and visible sectors}

In order to investigate what fraction of energy density is transferred to the hidden and visible sectors, we have to work out the moduli mass spectrum and the inflaton coupling to visible and hidden sector dof. This can be done starting to derive the canonical normalisation. The first step is to find a global minimum and then expand each modulus around its VEV:

$$
\tau_{i}=\left\langle\tau_{i}\right\rangle+\delta \tau_{i}, \quad \forall i=1, \ldots, h_{1,1},
$$

ending up with:

$$
\mathcal{L}=K_{i j} \partial_{\mu}\left(\delta \tau_{i}\right) \partial^{\mu}\left(\delta \tau_{j}\right)-\langle V\rangle-\frac{1}{2} V_{i j} \delta \tau_{i} \delta \tau_{j}+\mathcal{O}\left(\delta \tau^{3}\right)
$$

Writing the original moduli $\delta \tau_{i}$ in terms of the canonically normalised fields around the minimum $\delta \phi_{i}$ as:

$$
\delta \tau_{i}=\frac{1}{\sqrt{2}} C_{i j} \delta \phi_{j}
$$

the Lagrangian (4.2) takes the canonical form:

$$
\mathcal{L}=\frac{1}{2} \sum_{i=1}^{h_{1,1}} \partial_{\mu}\left(\delta \phi_{i}\right) \partial^{\mu}\left(\delta \phi_{i}\right)-\langle V\rangle-\frac{m_{i}^{2}}{2} \sum_{i=1}^{h_{1,1}} \delta \phi_{i}^{2},
$$

only if:

$$
C_{a i} K_{i j} C_{j b}=\delta_{a b} \quad \text { and } \quad \frac{1}{2} C_{a i} V_{i j} C_{j b}=m_{a}^{2} \delta_{a b}
$$

The two previous relations are satisfied if $C_{j a}$ and $m_{a}^{2}$ are, respectively, the eigenvectors and the eigenvalues of the mass-squared matrix $\left(M^{2}\right)_{i j} \equiv \frac{1}{2}\left(K^{-1}\right)_{i k} V_{k j}$.

In this way we have been able to derive the moduli mass-spectrum. Then the inflaton coupling to visible and hidden sector dof can be explicitly worked out by knowing the moduli dependence of the kinetic and mass terms of open string modes. Subsequently the moduli are expanded around 
their VEVs and then expressed in terms of the canonically normalised fields using (4.3). Following this procedure, in appendix A, we derived the moduli canonical normalisation, mass spectrum and couplings to all particles in the model for the case of BI and FI both in the geometric regime and at the quiver locus.

Let us outline here just the derivation of the moduli coupling to the massless gauge bosons of the field theory living on a stack of $D 7$-branes wrapping a 4-cycle whose volume is given by $\tau$ (which can be any of our moduli). We focus just on this case since this is the simplest derivation and, above all, in appendix $\mathrm{A}$ we show that the strongest moduli decay rates are to visible or hidden gauge bosons.

These couplings can be worked out from the moduli dependence of the tree-level gauge kinetic function [52], which in the case when $\tau$ is fixed in the geometric regime, is given by $4 \pi g^{-2}=\tau$. The kinetic terms read:

$$
\mathcal{L}_{\text {gauge }}=-\frac{\tau}{M_{P}} F_{\mu \nu} F^{\mu \nu}
$$

We then expand $\tau$ around its minimum and go to the canonically normalised field strength $G_{\mu \nu}$ defined as $G_{\mu \nu}=2 \sqrt{\tau} F_{\mu \nu}$ and obtain:

$$
\mathcal{L}_{\text {gauge }}=-\frac{1}{4} G_{\mu \nu} G^{\mu \nu}-\frac{\delta \tau}{4 M_{P}\langle\tau\rangle} G_{\mu \nu} G^{\mu \nu} .
$$

Now by substituting one of the expressions (4.3) for $\delta \tau$ in (A.28), we obtain the moduli couplings to the corresponding gauge bosons.

We notice that the expression of the gauge kinetic function changes in the case when $\tau$ shrinks down at the singularity. In fact in this case the tree-level bit depends on the axio-dilaton $S=$ $e^{-\phi}+i C_{0}$ and the Kähler modulus enters only at 1-loop in the presence of a non vanishing worldvolume flux $F$ 115:

$$
4 \pi g^{-2}=\operatorname{Re}(S)+h(F) \tau .
$$

Hence in this case, in order to derive the moduli couplings to the the corresponding gauge bosons, one has to work out the moduli mixing with the axio-dilaton $S$ (see appendix A).

Let us illustrate these claims in the explicit examples of BI and FI both in the geometric regime and at the quiver locus.

\subsection{Blow-up inflation}

Let us focus on the inflationary model described in section 2.2. We shall consider both the case when the cycle $\tau_{4}$ supporting the visible sector is stabilised in the geometric regime or at the quiver locus.

\subsubsection{Geometric Regime}

The canonical normalisation reads (see appendix A):

$$
\begin{aligned}
\delta \tau_{1} & \sim \mathcal{O}\left(\mathcal{V}^{2 / 3}\right) \delta \phi_{1}+\sum_{i=2}^{4} \mathcal{O}\left(\mathcal{V}^{1 / 6}\right) \delta \phi_{i} \sim \mathcal{O}\left(\mathcal{V}^{2 / 3}\right) \delta \phi_{1} \\
\delta \tau_{2} & \sim \mathcal{O}(1) \delta \phi_{1}+\mathcal{O}\left(\mathcal{V}^{1 / 2}\right) \delta \phi_{2}+\sum_{i=3}^{4} \mathcal{O}\left(\mathcal{V}^{-1 / 2}\right) \delta \phi_{i} \sim \mathcal{O}\left(\mathcal{V}^{1 / 2}\right) \delta \phi_{2} \\
\delta \tau_{3} & \sim \mathcal{O}(1) \delta \phi_{1}+\mathcal{O}\left(\mathcal{V}^{1 / 2}\right) \delta \phi_{3}+\sum_{i=2,4} \mathcal{O}\left(\mathcal{V}^{-1 / 2}\right) \delta \phi_{i} \sim \mathcal{O}\left(\mathcal{V}^{1 / 2}\right) \delta \phi_{3} \\
\delta \tau_{4} & \sim \mathcal{O}(1) \delta \phi_{1}+\mathcal{O}\left(\mathcal{V}^{1 / 2}\right) \delta \phi_{4}+\sum_{i=2}^{3} \mathcal{O}\left(\mathcal{V}^{-1 / 2}\right) \delta \phi_{i} \sim \mathcal{O}\left(\mathcal{V}^{1 / 2}\right) \delta \phi_{4}
\end{aligned}
$$


and the moduli mass spectrum looks like:

$$
m_{1}^{2} \simeq \frac{M_{P}^{2}}{\mathcal{V}^{3} \ln \mathcal{V}}, \quad \text { and } \quad m_{i}^{2} \simeq \frac{(2 \ln \mathcal{V})^{2}}{\mathcal{V}^{2}} M_{P}^{2}, \quad \forall i=2,3,4
$$

We point out that the volume-scaling of the canonical normalisation of the small blow-up modes $\delta \tau_{i}$, $i=2,3,4$, can be understood from a geometric point of view. In fact, each canonically normalised field $\delta \phi_{i}, i=2,3,4$, is mostly given by the corresponding blow-up mode with a power of $\mathcal{V}^{1 / 2}$, then the next mixing in a large volume expansion is with $\delta \tau_{1}$ which corresponds to the overall volume mode. Finally the mixing with all the other blow-up modes is further suppressed by a power of $\mathcal{V}^{-1 / 2}$. This suppression with respect to the mixing with the overall volume mode $\delta \tau_{1}$, is reflecting the geometrical separation in the internal space between the different localised singularities resolved by each different blow-up mode.

As we have seen in section 2.2.1, we need to fine-tune the coefficient of the string loop corrections in order to prevent them from spoiling the flatness of the inflationary potential. In addition, we shall consider two situations:

1. Inflaton 4-cycle $\tau_{2}$ wrapped just by the hidden sector $D 7$ stack;

2. Inflaton 4-cycle $\tau_{2}$ wrapped by both the visible and the hidden sector $D 7$ stack. More precisely, the hidden sector $D 7$-branes are wrapped around $\tau_{2}$ while the visible sector is wrapped around a combination of $\tau_{2}$ and $\tau_{4}$ with chiral intersections only on $\tau_{4}$.

Inflaton 4-cycle not wrapped by the visible sector

The brane set-up is (assuming that the tadpole-cancelation condition can be satisfied by an appropriate choice of background fluxes):

- $\tau_{1}$ is not wrapped by any brane;

- $\tau_{2}$ is wrapped by a hidden sector $D 7$-stack that undergoes gaugino-condensation;

- $\tau_{3}$ is wrapped by a hidden sector $D 7$-stack that undergoes gaugino-condensation;

- $\tau_{4}$ is wrapped by the visible sector stack of $D 7$-branes.

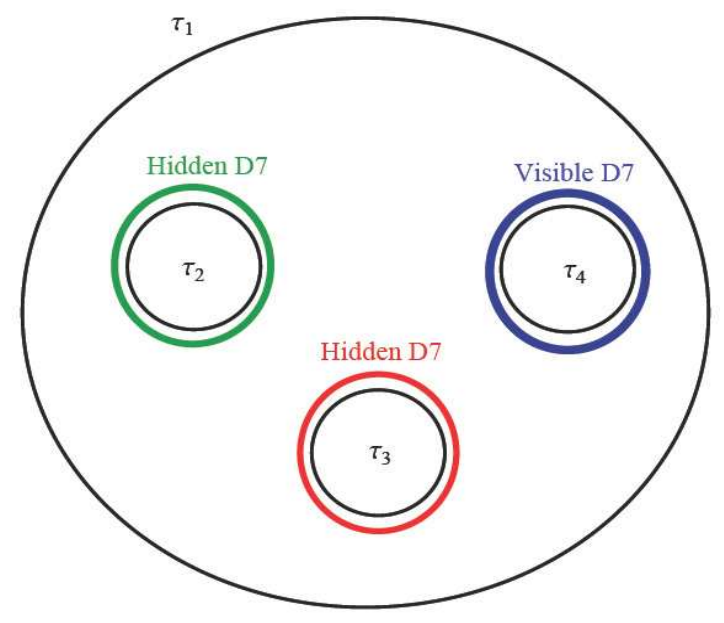

Figure 1: Brane set-up for BI in the geometric regime with the inflaton $\tau_{2}$ wrapped by a hidden sector. 
The order of magnitude of the moduli couplings to hidden gauge bosons living on $\tau_{2}$ and $\tau_{3}$, and to visible gauge bosons living on $\tau_{4}$ reads (see appendix $\mathrm{A}$ for a detailed derivation):

\begin{tabular}{c||c|c|c|c} 
& $\delta \phi_{1}$ & $\delta \phi_{2}$ & $\delta \phi_{3}$ & $\delta \phi_{4}$ \\
\hline \hline$\left(F_{\mu \nu}^{(2)} F_{(2)}^{\mu \nu}\right)$ & $\frac{1}{M_{P}}$ & $\frac{\mathcal{V}^{1 / 2}}{M_{P}}$ & $\frac{1}{\mathcal{V}^{1 / 2} M_{P}}$ & $\frac{1}{\mathcal{V}^{1 / 2} M_{P}}$ \\
\hline$\left(F_{\mu \nu}^{(3)} F_{(3)}^{\mu \nu}\right)$ & $\frac{1}{M_{P}}$ & $\frac{1}{\mathcal{V}^{1 / 2} M_{P}}$ & $\frac{\mathcal{V}^{1 / 2}}{M_{P}}$ & $\frac{1}{\mathcal{V}^{1 / 2} M_{P}}$ \\
\hline$\left(F_{\mu \nu}^{(4)} F_{(4)}^{\mu \nu}\right)$ & $\frac{1}{M_{P}}$ & $\frac{1}{\mathcal{V}^{1 / 2} M_{P}}$ & $\frac{1}{\mathcal{V}^{1 / 2} M_{P}}$ & $\frac{\mathcal{V}^{1 / 2}}{M_{P}}$
\end{tabular}

As studied in [19], at the end of inflation, due to the steepness of the potential, the inflaton $\tau_{2}$ stops oscillating just after two or three oscillations due to an extremely efficient non-perturbative particle production of $\delta \tau_{2}$ fluctuations. From the canonical normalisation (4.10), we then realise that our Universe will mostly be filled with $\delta \phi_{2}$-particles plus some $\delta \phi_{1}$ and fewer $\delta \phi_{3}$ and $\delta \phi_{4^{-}}$ particles. Therefore the energy density of the Universe is dominated by $\delta \phi_{2}$ whose decay will be responsible for reheating.

Then denoting as $g$ the visible gauge bosons and with $X_{2}$ and $X_{3}$ the hidden ones, we immediately realise that the coupling of $\delta \phi_{2}$ to $g g$ is suppressed by an inverse power of the overall volume with respect to its coupling to $X_{2} X_{2}$. Hence the inflaton will dump all its energy to hidden, instead of visible, sector dof. More precisely the first particles to decay are:

$$
\delta \phi_{2} \rightarrow X_{2} X_{2}, \quad \delta \phi_{3} \rightarrow X_{3} X_{3}, \quad \delta \phi_{4} \rightarrow g g .
$$

Later on also $\delta \phi_{1}$ decays to visible and hidden $d o f$ out of thermal equilibrium.

The only way to avoid the inflaton energy dumping to hidden sector dof is to forbid the decay of $\delta \phi_{2}$ to any hidden particle. In section 3 we have studied the dynamics of the hidden sector gauge theory and the relative mass spectrum. Moreover we focused on the simplest case of a rigid blow-up cycle invariant under the orientifold, not intersecting with any other cycle and not shrinking at the quiver locus. In this case we have found that the mass spectrum is strictly dependent on the way the $D 7$-branes get magnetised, finding some cases where all hidden sector particles get a mass $m$ greater then the inflaton mass $m_{2}: m \geq \Lambda>m_{2}$. This is the case when no gauge flux is turned on, or $F_{2} \neq 0$ but all matter fields get a string scale mass due to their Yukawa interaction with a scalar that takes an $\mathcal{O}\left(M_{s}\right)$ VEV via $D$-terms [49].

We shall therefore consider these cases where the decay of $\delta \phi_{2}$ to $X_{2} X_{2}$ is kinematically forbidden. As pointed out before, we stress again that the coupling of the hidden dof to the visible ones is too weak to reheat the visible sector via the decay of hidden to visible $d o f$. Then the first particles to decay are $\delta \phi_{3}$ and $\delta \phi_{4}$ but their decay does not give rise to any reheating since the energy density of the Universe is dominated by $\delta \phi_{2}$. Reheating takes place only later on when $\delta \phi_{2}$ decays to visible gauge bosons with total decay rate (see appendix A):

$$
\Gamma_{\delta \phi_{2} \rightarrow g g}^{T O T} \simeq(\ln \mathcal{V})^{3} \frac{M_{P}}{\mathcal{V}^{4}}
$$

The maximal reheating temperature for the visible sector in the approximation of sudden thermalisation can be worked out as follows:

$$
\frac{4\left(\Gamma_{\delta \phi_{2} \rightarrow g g}^{T O T}\right)^{2}}{3}=3 H^{2}=\left(\frac{g_{*}^{v i s} \pi^{2}}{30}\right) \frac{\left(T_{R H}^{m a x}\right)^{4}}{M_{P}^{2}} \Rightarrow T_{R H}^{\max }=\left(\frac{40}{g_{*}^{v i s} \pi^{2}}\right)^{1 / 4} \sqrt{\Gamma_{\delta \phi_{2} \rightarrow g g}^{T O T} M_{P}}
$$

where $g_{*}^{v i s} \simeq 200$ is the total number of relativistic dof in the thermal bath. Hence we end up with:

$$
T_{R H}^{\max } \simeq\left(\frac{(\ln \mathcal{V})^{3 / 2}}{2}\right) \frac{M_{P}}{\mathcal{V}^{2}} .
$$


We notice that for $\mathcal{V} \sim 10^{6-7}$ (as needed to match the COBE normalisation for the density fluctuations in BI), we would obtain $T_{R H}^{\max } \simeq 10^{6-8} \mathrm{GeV}$ for the ideal case when all the dof are relativistic. This is the largest temperature a visible sector can have in this particular scenario.

However, given that $\delta \phi_{2}$ couples to $g g$ and $X_{3} X_{3}$ with the same $\left(1 /\left(M_{P} \sqrt{\mathcal{V}}\right)\right)$-strength, the inflaton would decay at the same time to visible and hidden sector dof living on $\tau_{3}$. This might give rise to the problem of hidden sector dark matter overproduction due to the fact that $\delta \phi_{2}$ releases the same amount of entropy to both sectors. Let us see in detail the behaviour with respect to this problem of each realisation of the hidden sector outlined in section 3:

1. Pure SYM theory: the inflaton decay to hidden sector dof is kinematically forbidden, and so reheating is viable.

2. Pure SYM theory plus a massless $U(1)$ : the mass spectrum below $\Lambda$ consists of massless photons plus photini with $\mathcal{O}\left(M_{\text {soft }}\right)$ mass. From the derivation of the moduli decay rates in appendix A, we find that the branching ratios $\operatorname{read}^{9}$ :

$$
\begin{gathered}
B R\left(\delta \phi_{2} \rightarrow g g\right) \simeq B R\left(\delta \phi_{2} \rightarrow \bar{\varphi} \varphi\right) \simeq 42 \%, \\
B R\left(\delta \phi_{2} \rightarrow g \bar{\psi} \psi\right) \simeq B R\left(\delta \phi_{2} \rightarrow \bar{H} H\right) \simeq B R\left(\delta \phi_{2} \rightarrow X_{3} X_{3}\right) \simeq B R\left(\delta \phi_{2} \rightarrow \lambda_{\text {hid }}^{(3)} \lambda_{\text {hid }}^{(3)}\right) \simeq 4 \%, \\
B R\left(\delta \phi_{2} \rightarrow \text { other visible } d o f\right) \simeq B R\left(\delta \phi_{2} \rightarrow \delta \phi_{1} \delta \phi_{1}\right) \simeq 0 \% .
\end{gathered}
$$

Therefore we might overproduce dark matter since about 4 over 100 inflaton decays yield hidden gauginos. We point out that this dark matter would be produced directly from the inflaton decay given that hidden photons and photini cannot form a thermal bath. In addition in the presence of $N_{G}^{\text {hid }}>1$ additional massless $U(1)$ factor the situation becomes even worse since:

$$
\frac{B R\left(\delta \phi_{2} \rightarrow g g\right)}{B R\left(\delta \phi_{2} \rightarrow X_{3} X_{3}\right)} \simeq \frac{10}{N_{G}^{h i d}}
$$

Hence we would prefer to discard this case.

3. Pure SYM theory plus matter but no massless $U(1)$ : the mass spectrum below $\Lambda$ consists of massless pions plus massive condensates with $\mathcal{O}\left(M_{\text {soft }}\right)$ mass. From the results of appendix A, we find that the branching ratios read ${ }^{10}$ :

- For $N_{f}=2$

$$
\begin{gathered}
B R\left(\delta \phi_{2} \rightarrow g g\right) \simeq B R\left(\delta \phi_{2} \rightarrow \bar{\varphi} \varphi\right) \simeq 40 \%, \quad B R\left(\delta \phi_{2} \rightarrow \bar{\psi}_{\text {hid }}^{(3)} \psi_{\text {hid }}^{(3)}\right) \simeq 8 \%, \\
B R\left(\delta \phi_{2} \rightarrow g \bar{\psi} \psi\right) \simeq B R\left(\delta \phi_{2} \rightarrow \bar{H} H\right) \simeq 4 \% \\
B R\left(\delta \phi_{2} \rightarrow \pi_{3} \pi_{3}\right) \simeq B R\left(\delta \phi_{2} \rightarrow \varphi_{\text {hid }}^{(3)} \varphi_{\text {hid }}^{(3)}\right) \simeq 2 \% \\
B R\left(\delta \phi_{2} \rightarrow \text { other visible } \operatorname{dof}\right) \simeq B R\left(\delta \phi_{2} \rightarrow \delta \phi_{1} \delta \phi_{1}\right) \simeq 0 \%
\end{gathered}
$$

- For $N_{f}=5$

$$
\begin{gathered}
B R\left(\delta \phi_{2} \rightarrow \bar{\psi}_{\text {hid }}^{(3)} \psi_{\text {hid }}^{(3)}\right) \simeq 32 \%, \quad B R\left(\delta \phi_{2} \rightarrow g g\right) \simeq B R\left(\delta \phi_{2} \rightarrow \bar{\varphi} \varphi\right) \simeq 25 \%, \\
B R\left(\delta \phi_{2} \rightarrow \pi_{3} \pi_{3}\right) \simeq B R\left(\delta \phi_{2} \rightarrow \varphi_{\text {hid }}^{(3)} \varphi_{\text {hid }}^{(3)}\right) \simeq 6 \%, \\
B R\left(\delta \phi_{2} \rightarrow g \bar{\psi} \psi\right) \simeq B R\left(\delta \phi_{2} \rightarrow \bar{H} H\right) \simeq 3 \%, \\
B R\left(\delta \phi_{2} \rightarrow \text { other visible } d o f\right) \simeq B R\left(\delta \phi_{2} \rightarrow \delta \phi_{1} \delta \phi_{1}\right) \simeq 0 \% .
\end{gathered}
$$

\footnotetext{
${ }^{9}$ Here the visible sector particles are denoted as $\psi$ (for fermions), $H$ (for Higgs), $\lambda$ (for gauginos), $\varphi$ (for SUSY scalars) and $\tilde{H}$ (for Higgsinos) whereas $\lambda_{\text {hid }}^{(3)}$ denotes hidden gauginos on $\tau_{3}$.

${ }^{10}$ The hidden dof on $\tau_{3}$ are denoted as $\psi_{\text {hid }}^{(3)}$ (for massive fermionic condensates), $\varphi_{\text {hid }}^{(3)}$ (for massive scalar condensates) and $\pi_{3}$ (for massless pion-like mesons).
} 
Due to the large branching ratio of the inflaton decay to hidden massive fermionic condensates, hidden dark matter would be overproduced, and so we need to discard this case. On top of this, these particles will form a thermal bath due to their residual QCD-like interactions, and massless hidden pions could then be produced not just directly from the inflaton decay but also thermally.

Given that the hidden sector particles interact with the visible ones only gravitationally, two thermal baths will get formed with two different temperatures $T_{v i s}$ and $T_{h i d}$, with $T_{v i s} \neq T_{\text {hid }}$ but both of them of the same order of magnitude as $T_{R H}^{\max }$ given by (4.17). Then both the thermal baths will contribute to the expansion of the Universe given by eq. (2.36), where the number of hidden $d o f$ is given by: $g_{*}^{\text {hid }}=N_{f}^{2},{ }^{11}$ yielding too many new relativistic $d o f$ in contrast with the severe bounds from BBN, which allows only one relativistic species, i.e. $N_{f}=1$. Hence this is another reason why we need to discard this case.

4. Pure SYM theory plus matter and a massless $U(1)$ : this case has to be discarded due to the reasons outlined above.

We have seen that the only way to avoid the overproduction of hidden dark matter living on $\tau_{3}$ from the inflaton decay, is to forbid the decay of $\delta \phi_{2}$ to any hidden particle. Hence, like in the case of $\tau_{2}$, also $\tau_{3}$ has to support a pure $N=1 \mathrm{SYM}$ theory. We finally stress that the requirement of having a viable reheating sets severe constraints on the particular brane constructions realising the hidden sectors. For example, no hidden photon can be present in the effective field theory even for a brane wrapping the large cycle $\tau_{1}$ since $\delta \phi_{2}$ would also couple to those photons as $1 /\left(M_{P} \sqrt{\mathcal{V}}\right)$.

Inflaton 4-cycle wrapped by the visible sector

The brane set-up is (assuming that the tadpole-cancelation condition can be satisfied by an appropriate choice of background fluxes):

- $\tau_{1}$ is not wrapped by any brane;

- $\tau_{2}$ is wrapped by a hidden sector $D 7$-stack that undergoes gaugino-condensation plus the visible sector $D 7$-stack which has chiral intersections with $\tau_{4}$ but not with $\tau_{2}$;

- $\tau_{3}$ is wrapped by a hidden sector $D 7$-stack that undergoes gaugino-condensation;

- $\tau_{4}$ is wrapped by the visible sector stack of $D 7$-branes.

The moduli couplings to hidden gauge bosons living on $\tau_{2}$ and $\tau_{3}$, and to visible gauge bosons living on $\tau_{4}$ scale as:

\begin{tabular}{c||c|c|c|c} 
& $\delta \phi_{1}$ & $\delta \phi_{2}$ & $\delta \phi_{3}$ & $\delta \phi_{4}$ \\
\hline \hline$\left(F_{\mu \nu}^{(2)} F_{(2)}^{\mu \nu}\right)$ & $\frac{1}{M_{P}}$ & $\frac{\mathcal{V}^{1 / 2}}{M_{P}}$ & $\frac{1}{\mathcal{V}^{1 / 2} M_{P}}$ & $\frac{1}{\mathcal{V}^{1 / 2} M_{P}}$ \\
\hline$\left(F_{\mu \nu}^{(3)} F_{(3)}^{\mu \nu}\right)$ & $\frac{1}{M_{P}}$ & $\frac{1}{\mathcal{V}^{1 / 2} M_{P}}$ & $\frac{\mathcal{V}^{1 / 2}}{M_{P}}$ & $\frac{1}{\mathcal{V}^{1 / 2} M_{P}}$ \\
\hline$\left(F_{\mu \nu}^{(4)} F_{(4)}^{\mu \nu}\right)$ & $\frac{1}{M_{P}}$ & $\frac{\mathcal{V}^{1 / 2}}{M_{P}}$ & $\frac{1}{\mathcal{V}^{1 / 2} M_{P}}$ & $\frac{\mathcal{V}^{1 / 2}}{M_{P}}$
\end{tabular}

At the end of inflation there will be non-perturbative production of $\delta \tau_{2}$ excitations given that the field $\tau_{2}$ is the inflaton. From the canonical normalisation (4.10), we then realise that our

\footnotetext{
${ }^{11}$ The number of massless Goldstone bosons is given by the dimension of the broken global symmetry group which is $\operatorname{dim}\left(U\left(N_{f}\right)\right)=N_{f}^{2}$ (the $S U\left(N_{f}\right)$ factor comes from chiral symmetry breaking whereas the $U(1)$ factor comes from the breaking of the original $R$-symmetry).
} 


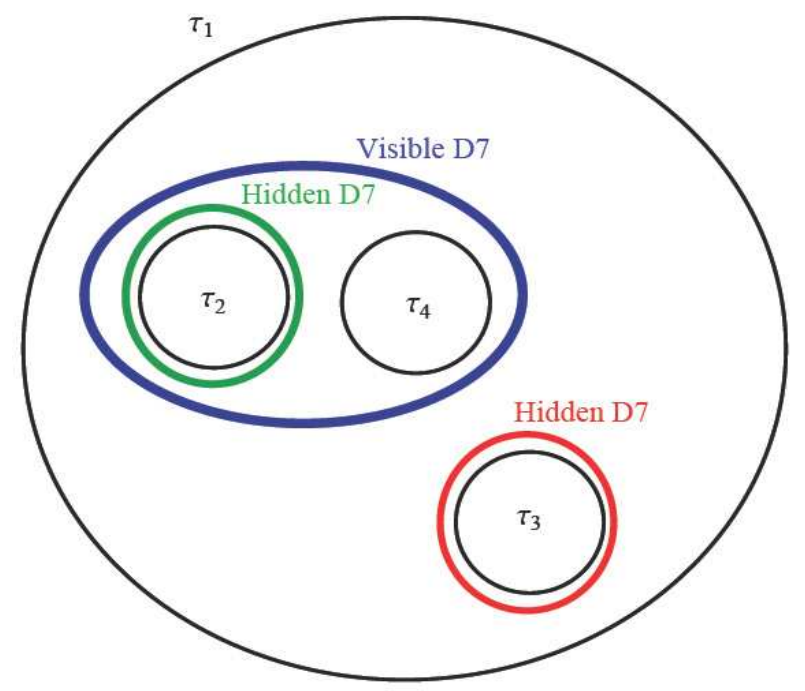

Figure 2: Brane set-up for BI in the geometric regime with the inflaton $\tau_{2}$ wrapped by the visible sector.

Universe will mostly be filled with $\delta \phi_{2}$-particles plus some $\delta \phi_{1}$ and fewer $\delta \phi_{3}$ and $\delta \phi_{4}$-particles. The inflaton $\delta \phi_{2}$ couples to visible gauge bosons $g$ and hidden gauge bosons $X_{2}$ with the same $1 / M_{s}$-strength while it couples much more weakly (as $1 /\left(M_{P} \sqrt{\mathcal{V}}\right)$ ) to the hidden gauge bosons living on $\tau_{3}$. Therefore $\delta \phi_{2}$ will decay at the same time to visible and hidden sector dof with the possible overproduction of dark matter particles from the hidden sector. More precisely the first particles to decay are:

$$
\delta \phi_{2} \rightarrow g g \text { or } X_{2} X_{2}, \quad \delta \phi_{3} \rightarrow X_{3} X_{3}, \quad \delta \phi_{4} \rightarrow g g .
$$

Only the decay of $\delta \phi_{2}$ gives rise to reheating since the energy density of the Universe is dominated by this particle. The maximal reheating temperature of the visible sector turns out to be (see appendix A]:

$$
\Gamma_{\delta \phi_{2} \rightarrow g g}^{T O T} \simeq 0.1(\ln \mathcal{V})^{3} \frac{M_{P}}{\mathcal{V}^{2}} \Rightarrow T_{R H}^{\max } \simeq 0.1(\ln \mathcal{V})^{3 / 2} \frac{M_{P}}{\mathcal{V}}
$$

We notice that for $\mathcal{V} \simeq 10^{6-7}$, we would obtain a rather high reheating temperature: $T_{R H}^{\max } \simeq$ $10^{12-13} \mathrm{GeV}$, that would allow any mechanism for the generation of the matter-antimatter asymmetry. Furthermore there is no gravitino problem since for $\mathcal{V} \simeq 10^{7}$ the gravitino would be heavier than $10 \mathrm{TeV}: m_{3 / 2} \simeq M_{P} / \mathcal{V} \simeq T_{R H}^{\max } \simeq 10^{11} \mathrm{GeV}$. Later on $\delta \phi_{1}$ decays out of thermal equilibrium to visible and hidden $d o f$.

Like in the case when the inflaton 4-cycle is not wrapped by the visible sector, we are again facing the same problem: dark matter overproduction due to the fact that $\delta \phi_{2}$ releases the same amount of entropy to the visible and the hidden sector living on $\tau_{2}$. Let us see in detail the behaviour of each possible realisation of the hidden sector outlined in section 3 :

- Pure SYM theory: the inflaton decay to hidden sector dof is kinematically forbidden, and so reheating is viable.

- Pure SYM theory plus a massless $U(1)$ : the mass spectrum below $\Lambda$ consists of massless photons plus photini with $\mathcal{O}\left(M_{\text {soft }}\right)$ mass. From the results of appendix $\mathrm{A}$, we find that the 
branching ratios read:

$$
\begin{gathered}
B R\left(\delta \phi_{2} \rightarrow g g\right) \simeq 50 \%, \quad B R\left(\delta \phi_{2} \rightarrow \bar{\varphi} \varphi\right) \simeq 25 \%, \\
B R\left(\delta \phi_{2} \rightarrow g \bar{\psi} \psi\right) \simeq B R\left(\delta \phi_{2} \rightarrow g \lambda \lambda\right) \simeq B R\left(\delta \phi_{2} \rightarrow X_{2} X_{2}\right) \simeq B R\left(\delta \phi_{2} \rightarrow \lambda_{h i d}^{(2)} \lambda_{h i d}^{(2)}\right) \simeq 5 \% \\
B R\left(\delta \phi_{2} \rightarrow \bar{H} H\right) \simeq B R\left(\delta \phi_{2} \rightarrow g \tilde{H} \tilde{H}\right) \simeq 2.5 \%, \quad B R\left(\delta \phi_{2} \rightarrow \delta \phi_{1} \delta \phi_{1}\right) \simeq 0 \% .
\end{gathered}
$$

Therefore we might overproduce dark matter since about 5 over 100 inflaton decays yield hidden gauginos. We point out that this dark matter would be produced directly from the inflaton decay given that hidden photons and photini cannot form a thermal bath. In addition in the presence of more than one additional massless $U(1)$ factor the situation becomes even worse hence we would prefer to discard this case.

- Pure SYM theory plus matter but no massless $U(1)$ : the mass spectrum below $\Lambda$ consists of massless pions plus massive condensates with $\mathcal{O}\left(M_{\text {soft }}\right)$ mass. The branching ratios read (see appendix A):

- For $N_{f}=2$

$$
\begin{gathered}
B R\left(\delta \phi_{2} \rightarrow g g\right) \simeq 50 \%, \quad B R\left(\delta \phi_{2} \rightarrow \bar{\varphi} \varphi\right) \simeq 25 \%, \\
B R\left(\delta \phi_{2} \rightarrow \bar{\psi}_{\text {hid }}^{(2)} \psi_{\text {hid }}^{(2)}\right) \simeq 10 \%, \quad B R\left(\delta \phi_{2} \rightarrow g \bar{\psi} \psi\right) \simeq B R\left(\delta \phi_{2} \rightarrow g \lambda \lambda\right) \simeq 5 \%, \\
B R\left(\delta \phi_{2} \rightarrow \bar{H} H\right) \simeq B R\left(\delta \phi_{2} \rightarrow g \tilde{H} \tilde{H}\right) \simeq 2.5 \%, \quad B R\left(\delta \phi_{2} \rightarrow \text { other hidden } d o f\right) \simeq 0 \% .
\end{gathered}
$$

- For $N_{f}=5$

$$
\begin{gathered}
B R\left(\delta \phi_{2} \rightarrow \bar{\psi}_{\text {hid }}^{(2)} \psi_{\text {hid }}^{(2)}\right) \simeq 40 \%, \quad B R\left(\delta \phi_{2} \rightarrow g g\right) \simeq 32 \%, \quad B R\left(\delta \phi_{2} \rightarrow \bar{\varphi} \varphi\right) \simeq 16 \%, \\
B R\left(\delta \phi_{2} \rightarrow g \bar{\psi} \psi\right) \simeq B R\left(\delta \phi_{2} \rightarrow g \lambda \lambda\right) \simeq 3 \%, \\
B R\left(\delta \phi_{2} \rightarrow \bar{H} H\right) \simeq B R\left(\delta \phi_{2} \rightarrow g \tilde{H} \tilde{H}\right) \simeq 2 \%, \\
B R\left(\delta \phi_{2} \rightarrow \pi_{2} \pi_{2}\right) \simeq B R\left(\delta \phi_{2} \rightarrow \varphi_{\text {hid }}^{(2)} \varphi_{\text {hid }}^{(2)}\right) \simeq 1 \%, \quad B R\left(\delta \phi_{2} \rightarrow \delta \phi_{1} \delta \phi_{1}\right) \simeq 0 \% .
\end{gathered}
$$

We immediately realise that we need to discard this case since we would overproduce hidden dark matter.

- Pure SYM theory plus matter and a massless $U(1)$ : this case has to be discarded due to the reasons outlined above.

We finally stress that we have seen also in this case how the requirement of having a viable reheating sets severe constraints on the hidden sector model building. Again the only clearly viable scenario is the one where the hidden sector on $\tau_{2}$ consists of just a pure SYM theory. On the other hand, in this case there is no constraint on the hidden sector wrapping $\tau_{3}$ or on another possible hidden sector wrapping $\tau_{1}$. Thus this model would allow the presence of hidden photons living on both a small and a large 4-cycle.

\subsubsection{Quiver Locus}

In this case the inflaton 4-cycle can never be wrapped by the visible sector since this is realised by $D 3$-branes at the $\tau_{4}$-singularity ${ }^{12}$. Therefore we need to focus only on the case of the inflaton

\footnotetext{
${ }^{12}$ The authors of [19] considered the very speculative case of the inflaton shrinking below the string scale at the end of inflation assuming that it is possible to derive such a model of inflation. The inflaton's energy should be transferred to closed strings which are created by the merging of two winding modes which appear in the theory when the cycle shrinks below the string scale. Afterwards these excited closed strings decay to KK gravitons which in turn decay to SM dof, in a way somehow similar to what happens at the end of brane inflation. However the realisation of inflation and the transition from the geometric to the singular regime is not very clear, and so we shall not consider this case.
} 
4-cycle wrapped by just the hidden sector undergoing gaugino condensation ${ }^{13}$. The resulting brane set-up is:

- $\tau_{1}$ is not wrapped by any brane;

- $\tau_{2}$ is wrapped by a hidden sector $D 7$-stack that undergoes gaugino-condensation;

- $\tau_{3}$ is wrapped by a hidden sector $D 7$-stack that undergoes gaugino-condensation;

- $\tau_{4}$ is shrunk down at the quiver locus and the visible sector is built via D3-branes at the singularity.

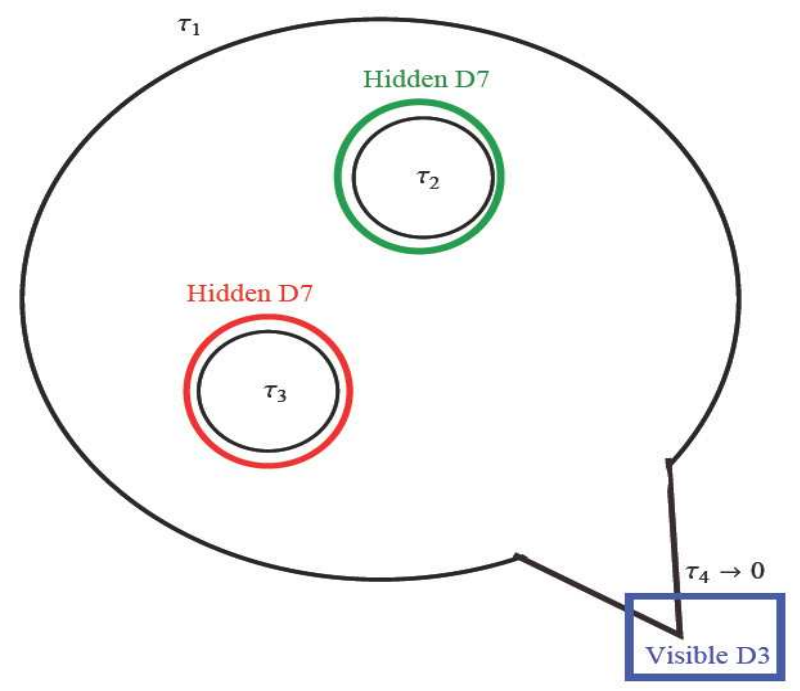

Figure 3: Brane set-up for BI at the quiver locus.

In this case the gauge kinetic function for the visible sector takes the form [15]:

$$
4 \pi g^{-2}=\operatorname{Re}(S)+h(F) \tau_{4},
$$

hence in order to work out the coupling of the inflaton $\tau_{2}$ to the visible gauge bosons, we have to derive its mixing with the real part of the axio-dilaton $s=\operatorname{Re}(S)$ and the other Kähler modulus $\tau_{4}$. The effective field theory at the quiver locus admits a Kähler potential which can be expanded around the singularity as (with $\hat{\xi} \equiv \xi / g_{s}^{3 / 2}=\xi / s^{3 / 2}$ ) 15:

$$
K=-2 \ln \left(\mathcal{V}^{\prime}+\frac{\hat{\xi}}{2}\right)+\lambda \frac{\tau_{4}^{2}}{\mathcal{V}^{\prime}}-\ln (2 s),
$$

with:

$$
\mathcal{V}^{\prime}=\alpha\left(\tau_{1}^{3 / 2}-\gamma_{2} \tau_{2}^{3 / 2}-\gamma_{3} \tau_{3}^{3 / 2}\right)
$$

The particular form of the Kähler potential (4.22) and $\left\langle\tau_{4}\right\rangle=0$ imply that at leading order there is no mixing between $\tau_{2}$ and $\tau_{4}$. In addition, at tree level, the Kähler potential takes a factorised form, and so $\tau_{2}$ does not mix with the axio-dilaton either. However $\tau_{2}$ mixes with $s$ due to $\alpha^{\prime}$ corrections

\footnotetext{
${ }^{13}$ It is understood that we assume that the coefficients of the string loop corrections are fine-tuned small in order not to spoil the flatness of the inflationary potential.
} 
to $K$ leading to the following general form of the canonical normalisation (see appendix $A$ for the detailed derivation):

$$
\begin{aligned}
\delta \tau_{1} & \sim \mathcal{O}\left(\mathcal{V}^{2 / 3}\right) \delta \phi_{1}+\sum_{i=2}^{3} \mathcal{O}\left(\mathcal{V}^{1 / 6}\right) \delta \phi_{i}+\mathcal{O}\left(\mathcal{V}^{1 / 6}\right) \delta \phi_{s} \sim \mathcal{O}\left(\mathcal{V}^{2 / 3}\right) \delta \phi_{1} \\
\delta \tau_{2} & \sim \mathcal{O}(1) \delta \phi_{1}+\mathcal{O}\left(\mathcal{V}^{1 / 2}\right) \delta \phi_{2}+\mathcal{O}\left(\mathcal{V}^{-1 / 2}\right) \delta \phi_{3}+\mathcal{O}\left(\mathcal{V}^{-1 / 2}\right) \delta \phi_{s} \sim \mathcal{O}\left(\mathcal{V}^{1 / 2}\right) \delta \phi_{2}, \\
\delta \tau_{3} & \sim \mathcal{O}(1) \delta \phi_{1}+\mathcal{O}\left(\mathcal{V}^{1 / 2}\right) \delta \phi_{3}+\mathcal{O}\left(\mathcal{V}^{-1 / 2}\right) \delta \phi_{2}+\mathcal{O}\left(\mathcal{V}^{-1 / 2}\right) \delta \phi_{s} \sim \mathcal{O}\left(\mathcal{V}^{1 / 2}\right) \delta \phi_{3}, \\
\delta \tau_{4} & \sim \mathcal{O}\left(\mathcal{V}^{1 / 2}\right) \delta \phi_{4} \\
\delta s & \sim \mathcal{O}\left(\mathcal{V}^{-1 / 2}\right) \delta \phi_{1}+\sum_{j=2}^{3} \mathcal{O}\left(\mathcal{V}^{-1}\right) \delta \phi_{j}+\mathcal{O}(1) \delta \phi_{s} \sim \mathcal{O}(1) \delta \phi_{s} .
\end{aligned}
$$

Then the volume scaling of the moduli couplings to hidden gauge bosons living on $\tau_{2}$ and $\tau_{3}$, and to visible gauge bosons at the $\tau_{4}$-singularity reads:

\begin{tabular}{c||c|c|c|c|c} 
& $\delta \phi_{1}$ & $\delta \phi_{2}$ & $\delta \phi_{3}$ & $\delta \phi_{4}$ & $\delta \phi_{s}$ \\
\hline \hline$\left(F_{\mu \nu}^{(2)} F_{(2)}^{\mu \nu}\right)$ & $\frac{1}{M_{P}}$ & $\frac{\mathcal{V}^{1 / 2}}{M_{P}}$ & $\frac{1}{\mathcal{V}^{1 / 2} M_{P}}$ & - & $\frac{1}{\mathcal{V}^{1 / 2} M_{P}}$ \\
\hline$\left(F_{\mu \nu}^{(3)} F_{(3)}^{\mu \nu}\right)$ & $\frac{1}{M_{P}}$ & $\frac{1}{\mathcal{V}^{1 / 2} M_{P}}$ & $\frac{\mathcal{V}^{1 / 2}}{M_{P}}$ & - & $\frac{1}{\mathcal{V}^{1 / 2} M_{P}}$ \\
\hline$\left(F_{\mu \nu}^{(4)} F_{(4)}^{\mu \nu}\right)$ & $\frac{1}{\mathcal{V}^{1 / 2} M_{P}}$ & $\frac{1}{\mathcal{V} M_{P}}$ & $\frac{1}{\mathcal{V} M_{P}}$ & $\frac{\mathcal{V}^{1 / 2}}{M_{P}}$ & $\frac{1}{M_{P}}$
\end{tabular}

At the end of inflation there will be non-perturbative production of $\delta \tau_{2}$ excitations given that the field $\tau_{2}$ is the inflaton. From the canonical normalisation (4.25), we then realise that our Universe will mostly be filled with $\delta \phi_{2}$-particles plus some $\delta \phi_{1}$ and fewer $\delta \phi_{3}$ and $\delta \phi_{s}$-particles.

This model is affected by the problem of the dumping of the inflaton energy to hidden, instead of visible, sector dof. In fact the coupling of $\delta \phi_{2}$ to $\left(F_{\mu \nu}^{(2)} F_{(2)}^{\mu \nu}\right)$ is stronger than the coupling to $\left(F_{\mu \nu}^{(3)} F_{(3)}^{\mu \nu}\right)$ which, in turn, is stronger than the coupling to the visible $\left(F_{\mu \nu}^{(4)} F_{(4)}^{\mu \nu}\right)$. The explanation of this different behaviour is the geometric separation between $\tau_{2}$ and $\tau_{3}$ and, on top of that, the sequestering of the visible sector at the $\tau_{4}$-singularity.

Thus the first particles to decay are $\delta \phi_{2} \rightarrow X_{2} X_{2}$ and $\delta \phi_{3} \rightarrow X_{3} X_{3}$. As we have seen in section 4.1.1, the only way to solve this problem is to forbid the decay of $\delta \phi_{2}$ to any hidden particle. This requirement forces us to consider brane constructions on both $\tau_{2}$ and $\tau_{3}$ that yield just a pure SYM theory with no matter that develops a mass gap, so that the decay of $\delta \phi_{2}$ to $X_{2} X_{2}$ or $X_{3} X_{3}$ is kinematically forbidden. Then the first particle to decay is $\delta \phi_{s} \rightarrow g g$ but without giving rise to any reheating since the energy density of the Universe is dominated by the inflaton field $\delta \phi_{2}$ which has not decayed yet. Reheating takes place only later on when $\delta \phi_{2}$ decays to $g g$ producing the following maximal reheating temperature for the visible sector:

$$
\Gamma_{\delta \phi_{2} \rightarrow g g}^{T O T} \simeq(\ln \mathcal{V})^{3} \frac{M_{P}}{\mathcal{V}^{5}} \Rightarrow T_{R H}^{\max } \simeq\left(\frac{(\ln \mathcal{V})^{3 / 2}}{2}\right) \frac{M_{P}}{\mathcal{V}^{5 / 2}}
$$

We notice that for $\mathcal{V} \simeq 10^{6}$, we would obtain $T_{R H}^{\max } \simeq 5 \cdot 10^{4} \mathrm{GeV}$ but for $\mathcal{V} \simeq 10^{7}$, we would find $T_{R H}^{\max } \simeq 10^{2} \mathrm{GeV}$. However this reheating temperature is quite higher than $T_{B B N} \simeq 1 \mathrm{MeV}$, and so it would not give rise to any problem if the matter-antimatter asymmetry could be realised in a non-thermal/thermal way. Note that baryogenesis is still possible at very low scales if R-parity violating interactions are introduced [53. We finally point out that this model does not allow the presence of any hidden photon since the coupling of $\delta \phi_{2}$ to possible hidden photons living on the large cycle $\tau_{1}$ would scale as $1 /\left(M_{P} \sqrt{\mathcal{V}}\right)$, and so it would be stronger than the coupling to visible dof on $\tau_{4}$. 


\subsection{Fibre Inflation}

Let us focus on the inflationary model described in section 2.3 considering both the case when the cycle $\tau_{4}$ supporting the visible sector is stabilised in the geometric regime or at the quiver locus.

\subsubsection{Geometric Regime}

The canonical normalisation reads (see appendix A):

$$
\begin{aligned}
\delta \tau_{1} & \sim \sum_{i=1}^{2} \mathcal{O}\left(\mathcal{V}^{2 / 3}\right) \delta \phi_{i}+\sum_{j=3}^{4} \mathcal{O}\left(\mathcal{V}^{1 / 6}\right) \delta \phi_{j} \sim \sum_{i=1}^{2} \mathcal{O}\left(\mathcal{V}^{2 / 3}\right) \delta \phi_{i} \\
\delta \tau_{2} & \sim \sum_{i=1}^{2} \mathcal{O}\left(\mathcal{V}^{2 / 3}\right) \delta \phi_{i}+\sum_{j=3}^{4} \mathcal{O}\left(\mathcal{V}^{1 / 6}\right) \delta \phi_{j} \sim \sum_{i=1}^{2} \mathcal{O}\left(\mathcal{V}^{2 / 3}\right) \delta \phi_{i} \\
\delta \tau_{3} & \sim \mathcal{O}\left(c^{-1 / 2} \mathcal{V}^{-1 / 3}\right) \delta \phi_{1}+\mathcal{O}(1) \delta \phi_{2}+\mathcal{O}\left(\mathcal{V}^{1 / 2}\right) \delta \phi_{3}+\mathcal{O}\left(\mathcal{V}^{-1 / 2}\right) \delta \phi_{4} \sim \mathcal{O}\left(\mathcal{V}^{1 / 2}\right) \delta \phi_{3} \\
\delta \tau_{4} & \sim \mathcal{O}\left(c^{-1 / 2} \mathcal{V}^{-1 / 3}\right) \delta \phi_{1}+\mathcal{O}(1) \delta \phi_{2}+\mathcal{O}\left(\mathcal{V}^{-1 / 2}\right) \delta \phi_{3}+\mathcal{O}\left(\mathcal{V}^{1 / 2}\right) \delta \phi_{4} \sim \mathcal{O}\left(\mathcal{V}^{1 / 2}\right) \delta \phi_{4}
\end{aligned}
$$

and the moduli mass spectrum looks like (with $\left\langle\tau_{1}\right\rangle=c \mathcal{V}^{2 / 3}$ ):

$$
m_{1}^{2} \simeq \frac{M_{P}^{2}}{c^{1 / 2} \mathcal{V}^{10 / 3}}, \quad m_{2}^{2} \simeq \frac{M_{P}^{2}}{\mathcal{V}^{3} \ln \mathcal{V}}, \quad \text { and } \quad m_{i}^{2} \simeq \frac{(2 \ln \mathcal{V})^{2}}{\mathcal{V}^{2}} M_{P}^{2}, \quad \forall i=3,4
$$

As shown in appendix A, inverting the exact form of (4.30) and (4.31), it turns out that $\delta \phi_{2}$ is a particular combination of $\delta \tau_{1}$ and $\delta \tau_{2}$ which corresponds to the overall volume, whereas $\delta \phi_{1}$ is a direction orthogonal to this one which is fixed only at subleading order by string loops. This is why, as can be seen from (4.34), $\delta \phi_{1}$ is lighter than $\delta \phi_{2}$, and plays the rôle of the inflaton. We also stress that the canonical normalisation for a K3 fibration and a Swiss-cheese Calabi-Yau has the same volume scaling once we identify $\delta \phi_{2}$ as the large overall volume mode. The brane set-up is:

- $\tau_{1}$ is wrapped by a hidden sector $D 7$-stack which generates string loops;

- $\tau_{2}$ is wrapped by a hidden sector $D 7$-stack which generates string loops;

- $\tau_{3}$ is wrapped by a hidden sector $D 7$-stack that undergoes gaugino-condensation;

- $\tau_{4}$ is wrapped by the visible sector stack of $D 7$-branes.

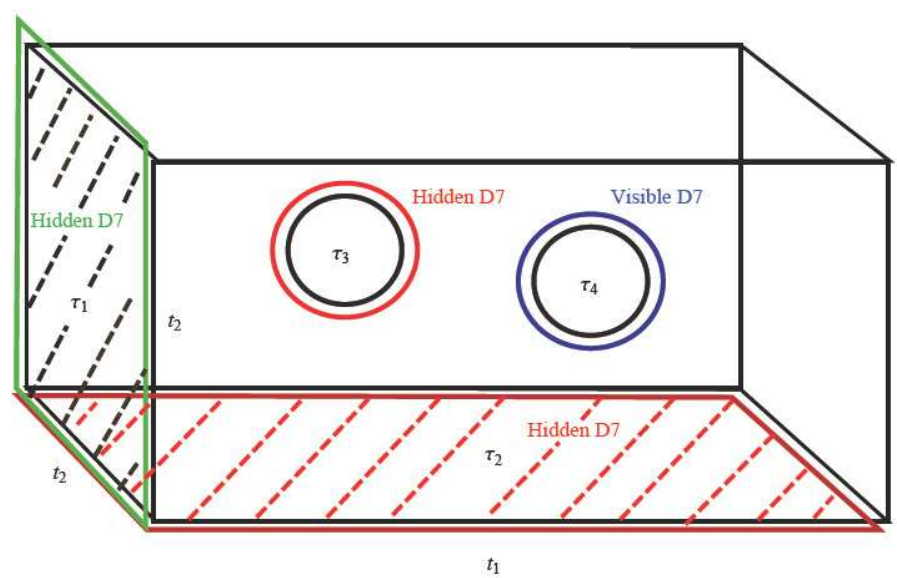

Figure 4: Brane set-up for FI in the geometric regime. 
The volume scaling of the moduli couplings to visible gauge bosons living on $\tau_{4}$, and to hidden gauge bosons living on $\tau_{1}, \tau_{2}$ and $\tau_{3}$ reads:

\begin{tabular}{c||c|c|c|c} 
& $\delta \phi_{1}$ & $\delta \phi_{2}$ & $\delta \phi_{3}$ & $\delta \phi_{4}$ \\
\hline \hline$\left(F_{\mu \nu}^{(1,2)} F_{(1,2)}^{\mu \nu}\right)$ & $\frac{1}{M_{P}}$ & $\frac{1}{M_{P}}$ & $\frac{1}{\mathcal{V}^{1 / 2} M_{P}}$ & $\frac{1}{\mathcal{V}^{1 / 2} M_{P}}$ \\
\hline$\left(F_{\mu \nu}^{(3)} F_{(3)}^{\mu \nu}\right)$ & $\frac{c^{-1 / 2}}{\mathcal{V}^{1 / 3} M_{P}}$ & $\frac{1}{M_{P}}$ & $\frac{\mathcal{V}^{1 / 2}}{M_{P}}$ & $\frac{1}{\mathcal{V}^{1 / 2} M_{P}}$ \\
\hline$\left(F_{\mu \nu}^{(4)} F_{(4)}^{\mu \nu}\right)$ & $\frac{c^{-1 / 2}}{\mathcal{V}^{1 / 3} M_{P}}$ & $\frac{1}{M_{P}}$ & $\frac{1}{\mathcal{V}^{1 / 2} M_{P}}$ & $\frac{\mathcal{V}^{1 / 2}}{M_{P}}$
\end{tabular}

As we have pointed out before, the inflaton $\chi$ is a particular combination of $\tau_{1}$ and $\tau_{2}$ orthogonal to the overall volume ( $a$ and $b$ are $\mathcal{O}(1)$ constants):

$$
\chi=a \ln \tau_{1}+b \ln \tau_{2}
$$

and so at the end of inflation there will be non-perturbative production of $\delta \chi$ excitations. Given that the shape of the inflationary potential for FI is much less steep than the one for BI, we expect a different behaviour for preheating without such a violent non-perturbative production of inflaton inhomogeneities. It might even be that in this case all the dynamics is purely perturbative. The canonical normalisation around the minimum for $\chi$ is given by:

$$
\delta \chi \sim \mathcal{O}(1) \delta \phi_{1}+\mathcal{O}\left(c^{-1 / 2} \mathcal{V}^{-1 / 3}\right) \delta \phi_{2}+\sum_{i=3}^{4} \mathcal{O}\left(\mathcal{V}^{-1 / 2}\right) \delta \phi_{i}
$$

where the subleading mixing with $\delta \phi_{2}$ is introduced by the string loop corrections to the Kähler potential (2.26). From the canonical normalisation eq. 4.36), we then realise that our Universe will mostly be filled with $\delta \phi_{1}$-particles plus some $\delta \phi_{2}$ and fewer $\delta \phi_{3}$ and $\delta \phi_{4}$-particles.

Then denoting as $g$ the visible gauge bosons and with $X_{1}, X_{2}$ and $X_{3}$ the hidden sector ones, the first particles to decay are $\delta \phi_{3}$ and $\delta \phi_{4}: \delta \phi_{3} \rightarrow X_{3} X_{3}$ and $\delta \phi_{4} \rightarrow g g$. However these decays do not give rise to any reheating since the energy density of the Universe is dominated by $\delta \phi_{1}$. This is also the case when the $\delta \phi_{2}$ particles decay to all the gauge bosons in the theory at the same time. Finally also $\delta \phi_{1}$ decays but no reheating takes place in the visible sector since the inflaton coupling to the hidden gauge bosons $X_{1}$ and $X_{2}$ is stronger than the one to visible gauge bosons and $X_{3}$ since (see appendix A):

$$
\frac{\Gamma_{\delta \phi_{1} \rightarrow g g}}{\Gamma_{\delta \phi_{1} \rightarrow X_{1} X_{1}}} \sim\left(\frac{0.01}{(\ln \mathcal{V})^{2}}\right) \frac{1}{c \mathcal{V}^{2 / 3}}=\left(\frac{0.01}{(\ln \mathcal{V})^{2}}\right) \frac{1}{\left\langle\tau_{1}\right\rangle} \ll 1
$$

Thus reheating turns out to be a problem in this model where the inflaton dumps all its energy to hidden, instead of visible, dof. There are two possible solutions to this problem:

1. Forbid the inflaton decay to hidden sector dof.

If all the hidden sector dof are heavier than the inflaton, then the inflaton decay to these particles is kinematically forbidden. In section 4.1, we have seen that this can indeed be the case if the hidden sector consists of a pure $N=1 \mathrm{SYM}$ theory that develops a mass gap. Nonetheless, in this case, the hidden sector supported by $\tau_{1}$ and $\tau_{2}$ is only responsible to source the string loop corrections that generate the inflationary potential, and so it does not have to undergo gaugino condensation. Hence it might have a much more complicated gauge group structure and particle content with several particles that would naturally be lighter than the inflaton. However there is nothing against having non-perturbative effects 
supported on $\tau_{1}$ and $\tau_{2}$ since these two cycles are stabilised large, and so in a regime where the non-perturbative corrections are subleading with respect to the string loops and do not modify the moduli stabilisation picture. We shall therefore assume that it is indeed possible to render all the hidden sector $d o f$ on $\tau_{1}$ and $\tau_{2}$ heavier than the inflaton, for example considering a pure SYM theory living on these cycles.

We have then to consider a pure SYM theory also on $\tau_{3}$ otherwise, as discussed in section 4.1, too much hidden sector dark matter would be produced by the simultaneous inflaton decay to visible and hidden $d o f$ living on $\tau_{3}$. Under all these constraints on the hidden sector model building, reheating can take place leading to the formation of a visible sector thermal bath with maximal temperature:

$$
\Gamma_{\delta \phi_{1} \rightarrow g g}^{T O T} \simeq\left(\frac{5 \cdot 10^{-4}}{\ln \mathcal{V}}\right) \frac{M_{P}}{c^{7 / 4} \mathcal{V}^{17 / 3}} \Rightarrow T_{R H}^{\max } \simeq\left(\frac{0.01}{(\ln \mathcal{V})^{1 / 2}}\right) \frac{M_{P}}{c^{7 / 8} \mathcal{V}^{17 / 6}}
$$

In the case of $\mathrm{FI}$ the requirement of matching the COBE normalisation for the density fluctuations sets $\mathcal{V} \simeq 10^{3-4}$, and using the same parameters of [21], $c \simeq 5 \cdot 10^{-2}$. For these values we would obtain a maximal reheating temperature of the order $T_{R H}^{\max } \simeq 5 \cdot 10^{5-8} \mathrm{GeV}$. We finally stress that this model does not allow the presence of any light hidden photon.

2. Locate the visible sector on $\tau_{1}$.

Another possible way-out is to change our initial brane set-up assuming that the visible sector is located on $\tau_{1}$ without any need to wrap $D 7$-branes around $\tau_{4}$. The first problem that one encounters with this set-up, is that the K3 fiber is not a rigid cycle and so one has to worry about how to fix the $D 7$-brane deformation moduli that would give rise to unwanted matter in the adjoint representation. Here we shall assume that these moduli can be fixed by the use of background fluxes. The second problem is the order of magnitude of the visible sector gauge coupling:

$$
\alpha_{1}=\frac{g_{1}^{2}}{4 \pi}=\frac{1}{\left\langle\tau_{1}\right\rangle}=\frac{1}{c \mathcal{V}^{2 / 3}},
$$

which for large volume would clearly become too small. However in the case of FI the volume is not too large: $\mathcal{V} \simeq 10^{4}$. In addition at the end of inflation the $\mathrm{K} 3$ fibre sits at a small size $\left\langle\tau_{1}\right\rangle \sim \mathcal{O}(10)$ (for $c \simeq 5 \cdot 10^{-2}$ ) which reproduces the correct order of magnitude of $\alpha_{1}$. Hence we shall assume that it is indeed possible to wrap the visible sector around $\tau_{1}$. Notice that in this case the stack of $D 7$-branes wrapped around $\tau_{2}$ would intersect with the visible sector stack wrapped around $\tau_{1}$. Hence there would be gauge interactions among the particles living on $\tau_{1}$ and $\tau_{2}$. Thus $\tau_{2}$ is not supporting a hidden sector but a different gauge group of the visible sector. The gauge coupling of this additional force is given by (with $\mathcal{V} \simeq \alpha \sqrt{\tau_{1}} \tau_{2}$ ):

$$
\alpha_{2}=\frac{g_{2}^{2}}{4 \pi}=\frac{1}{\tau_{2}}=\frac{\alpha \sqrt{\tau_{1}}}{\mathcal{V}}=\frac{\alpha c^{1 / 2}}{\mathcal{V}^{2 / 3}} \Leftrightarrow \frac{\alpha_{1}}{\alpha_{2}}=\frac{1}{\alpha c^{3 / 2}}
$$

and so for $c \simeq 5 \cdot 10^{-2}$ and $\alpha \simeq 0.1$ (as for typical Calabi-Yau examples [25]), the ratio $\alpha_{1} / \alpha_{2} \simeq 10^{3}$ takes the same order of magnitude of the ratio between the electromagnetic and the weak gauge coupling: $\alpha_{E M} / \alpha_{W} \simeq 5 \cdot 10^{3}$. Notice that the presence of at least two different visible sector gauge groups does not allow to build a GUT theory with a unified gauge group unless the gauge bosons on $\tau_{2}$ decouple from the effective field theory getting an $\mathcal{O}\left(M_{s}\right)$ mass.

Therefore the inflaton couples to visible gauge bosons living on $\tau_{1}$ and $\tau_{2}$ with the same $1 / M_{P}$ strength. In addition, in the presence of hidden massless pions living on $\tau_{3}, \delta \phi_{1}$ would also couple to these particles as $1 / M_{P}$. Hence the inflaton decay would yield too many new relativistic dof or it would overproduce hidden dark matter if the hidden pions on $\tau_{3}$ get 
massive via a low-energy Higgs-like mechanism. On the other hand, $\delta \phi_{1}$ would couple to hidden photons on $\tau_{3}$ as $1 /\left(M_{P} \mathcal{V}^{1 / 3}\right)$, so more weakly than its coupling to visible gauge bosons. Therefore the presence of an additional massless $U(1)$ factor on $\tau_{3}$ would not induce any problem. Assuming that the hidden sector on $\tau_{3}$ is a pure SYM theory with possibly the presence of an additional massless $U(1)$ factor, the visible sector can be safely reheated to the temperature:

$$
\Gamma_{\delta \phi_{1} \rightarrow g g}^{T O T} \simeq 0.05 \frac{M_{P}}{c^{3 / 4} \mathcal{V}^{5}} \Rightarrow T_{R H}^{\max } \simeq 0.1 \frac{M_{P}}{c^{3 / 8} \mathcal{V}^{5 / 2}}
$$

For $\mathcal{V} \simeq 10^{3-4}$ and $c \simeq 5 \cdot 10^{-2}$, we would obtain $T_{R H}^{\max } \simeq 10^{8-10} \mathrm{GeV}$. We finally stress that this model would allow the presence of hidden photons living on the small cycle $\tau_{3}$.

\subsubsection{Quiver Locus}

In this case the visible sector 4-cycle is shrunk down at the singularity and the resulting brane set-up is:

- $\tau_{1}$ is wrapped by a hidden sector $D 7$-stack which generates string loops;

- $\tau_{2}$ is wrapped by a hidden sector $D 7$-stack which generates string loops;

- $\tau_{3}$ is wrapped by a hidden sector $D 7$-stack that undergoes gaugino-condensation;

- $\tau_{4}$ is shrunk down at the quiver locus and the visible sector is built via D3-branes at the singularity.

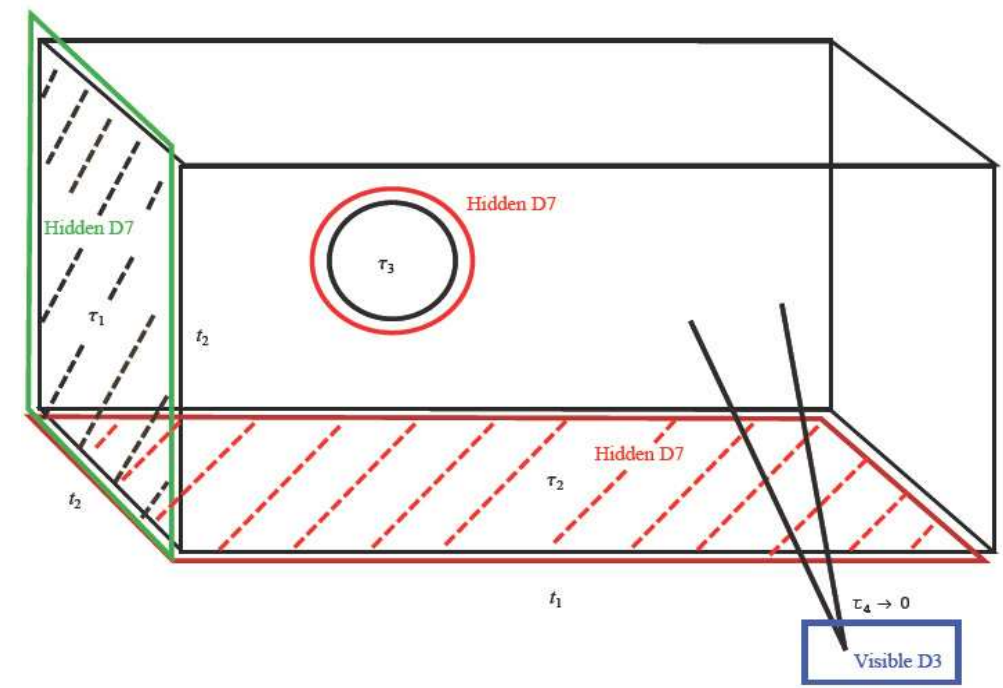

Figure 5: Brane set-up for FI at the quiver locus.

As we have seen in section 4.1.2, the particular form of the Kähler potential and $\left\langle\tau_{4}\right\rangle=0$ imply that at leading order there is no mixing between $\tau_{4}$ and the other moduli, leading to the following 
canonical normalisation:

$$
\begin{aligned}
\delta \tau_{1} & \sim \sum_{j=1}^{2} \mathcal{O}\left(\mathcal{V}^{2 / 3}\right) \delta \phi_{j}+\mathcal{O}\left(\mathcal{V}^{1 / 6}\right) \delta \phi_{3}+\mathcal{O}\left(\mathcal{V}^{1 / 6}\right) \delta \phi_{s} \sim \sum_{j=1}^{2} \mathcal{O}\left(\mathcal{V}^{2 / 3}\right) \delta \phi_{j} \\
\delta \tau_{2} & \sim \sum_{j=1}^{2} \mathcal{O}\left(\mathcal{V}^{2 / 3}\right) \delta \phi_{j}+\mathcal{O}\left(\mathcal{V}^{1 / 6}\right) \delta \phi_{3}+\mathcal{O}\left(\mathcal{V}^{1 / 6}\right) \delta \phi_{s} \sim \sum_{j=1}^{2} \mathcal{O}\left(\mathcal{V}^{2 / 3}\right) \delta \phi_{j} \\
\delta \tau_{3} & \sim \mathcal{O}\left(c^{-1 / 2} \mathcal{V}^{-1 / 3}\right) \delta \phi_{1}+\mathcal{O}(1) \delta \phi_{2}+\mathcal{O}\left(\mathcal{V}^{1 / 2}\right) \delta \phi_{3}+\mathcal{O}\left(\mathcal{V}^{-1 / 2}\right) \delta \phi_{s} \sim \mathcal{O}\left(\mathcal{V}^{1 / 2}\right) \delta \phi_{3}, \\
\delta \tau_{4} & \sim \mathcal{O}\left(\mathcal{V}^{1 / 2}\right) \delta \phi_{4} \\
\delta S & \sim \mathcal{O}\left(c^{-1 / 2} \mathcal{V}^{-5 / 6}\right) \delta \phi_{1}+\mathcal{O}\left(\mathcal{V}^{-1 / 2}\right) \delta \phi_{2}+\mathcal{O}\left(\mathcal{V}^{-1}\right) \delta \phi_{3}+\mathcal{O}(1) \delta \phi_{s} \sim \mathcal{O}(1) \delta \phi_{s}
\end{aligned}
$$

Then the moduli couplings to visible gauge bosons at the $\tau_{4}$-singularity, and to hidden gauge bosons living on $\tau_{1}, \tau_{2}$ and $\tau_{3}$ scale as:

\begin{tabular}{c||c|c|c|c|c} 
& $\delta \phi_{1}$ & $\delta \phi_{2}$ & $\delta \phi_{3}$ & $\delta \phi_{4}$ & $\delta \phi_{s}$ \\
\hline \hline$\left(F_{\mu \nu}^{(1,2)} F_{(1,2)}^{\mu \nu}\right)$ & $\frac{1}{M_{P}}$ & $\frac{1}{M_{P}}$ & $\frac{1}{\mathcal{V}^{1 / 2} M_{P}}$ & - & $\frac{1}{\mathcal{V}^{1 / 2} M_{P}}$ \\
\hline$\left(F_{\mu \nu}^{(3)} F_{(3)}^{\mu \nu}\right)$ & $\frac{c^{-1 / 2}}{\mathcal{V}^{1 / 3} M_{P}}$ & $\frac{1}{M_{P}}$ & $\frac{\mathcal{V}^{1 / 2}}{M_{P}}$ & - & $\frac{1}{\mathcal{V}^{1 / 2} M_{P}}$ \\
\hline$\left(F_{\mu \nu}^{(4)} F_{(4)}^{\mu \nu}\right)$ & $\frac{c^{-1 / 2}}{\mathcal{V}^{5 / 6} M_{P}}$ & $\frac{1}{\mathcal{V}^{1 / 2} M_{P}}$ & $\frac{1}{\mathcal{V} M_{P}}$ & $\frac{\mathcal{V}^{1 / 2}}{M_{P}}$ & $\frac{1}{M_{P}}$
\end{tabular}

At the end of inflation there will be non-perturbative production of $\delta \chi$ excitations. The canonical normalisation around the minimum for $\chi$ is given by:

$$
\delta \chi \sim \mathcal{O}(1) \delta \phi_{1}+\mathcal{O}\left(c^{-1 / 2} \mathcal{V}^{-1 / 3}\right) \delta \phi_{2}+\mathcal{O}\left(\mathcal{V}^{-1 / 2}\right) \delta \phi_{3}+\mathcal{O}\left(\mathcal{V}^{-1 / 2}\right) \delta \phi_{s},
$$

where the subleading mixing with $\delta \phi_{2}$ is introduced by the string loop corrections to the Kähler potential (2.26). From the canonical normalisation (4.47), we then realise that our Universe will mostly be filled with $\delta \phi_{1}$-particles plus some $\delta \phi_{2}$ and fewer $\delta \phi_{3}$ and $\delta \phi_{s}$-particles.

Then denoting as $g$ the visible sector gauge bosons and with $X_{1}, X_{2}$ and $X_{3}$ the hidden sector ones, the first decay is $\delta \phi_{3} \rightarrow X_{3} X_{3}$ and later on $\delta \phi_{s} \rightarrow g g$. However these decays do not give rise to any reheating since the energy density of the Universe is dominated by $\delta \phi_{1}$ which has not decayed yet. This is also the case when the $\delta \phi_{2}$ particles decay to all the hidden sector gauge bosons in the theory at the same time.

Later on the inflaton dumps all its energy to hidden, instead of visible, gauge bosons due to the fact that the coupling of $\delta \phi_{1}$ to $\left(F_{\mu \nu}^{(1,2)} F_{(1,2)}^{\mu \nu}\right)$ is stronger than the coupling to $\left(F_{\mu \nu}^{(3)} F_{(3)}^{\mu \nu}\right)$ which, in turn, is even stronger than the coupling to the visible $\left(F_{\mu \nu}^{(4)} F_{(4)}^{\mu \nu}\right)$. Hence in this model the reheating of the visible sector turns out to be a problem. We stress that we cannot try to overcome this problem as we did in the geometric regime case, where we assumed a different set-up with the visible sector localised on $\tau_{1}$. In fact, in this case, $\tau_{1}$ cannot shrink to zero size since the all volume of the Calabi-Yau would shrink to zero size. However we can imagine a brane construction such that all the hidden sectors in the model consist of a pure SYM theory that develops a mass gap. In this way all the hidden gauge bosons and gauginos condense and become heavier than $\delta \phi_{1}$ forbidding the corresponding decays.

Under these assumptions, the maximal reheating temperature due to the $\delta \phi_{1}$ decay to visible gauge bosons turns out to be:

$$
\Gamma_{\delta \phi_{1} \rightarrow g g}^{T O T} \simeq\left(\frac{5 \cdot 10^{-4}}{\ln \mathcal{V}}\right) \frac{M_{P}}{c^{7 / 4} \mathcal{V}^{20 / 3}} \Rightarrow T_{R H}^{\max } \simeq\left(\frac{0.01}{(\ln \mathcal{V})^{1 / 2}}\right) \frac{M_{P}}{c^{7 / 8} \mathcal{V}^{10 / 3}}
$$


For $\mathcal{V} \simeq 10^{3-4}$ and $c \simeq 5 \cdot 10^{-2}$, we would obtain $T_{R H}^{\max } \simeq 10^{4-7} \mathrm{GeV}$. As far as hidden photons are concerned, we found that the requirement of having a viable reheating of the visible sector automatically forbids their presence in the effective field theory both for BI and for FI when the cycle supporting the visible sector is shrunk down at the singularity.

\section{Discussion and Conclusions}

Once the inflaton has completely decayed into visible sector $d o f$, the system evolves to a complete thermalisation and the thermal bath generates a moduli-dependent finite temperature scalar potential $^{14}$. The behaviour of this potential for an arbitrary LVS has been studied in [54] where it was found that the finite temperature corrections do not develop any new minimum, and so they cannot induce any phase transition, but they can still lead the system to a dangerous decompactification limit. The computation of the decompactification temperature for LVS gives 54:

$$
T_{\max } \simeq \frac{M_{P}}{\mathcal{V}^{3 / 4}},
$$

and this is valid for all the different scenarios that we have studied here. This temperature sets the maximal temperature of our Universe, and so one has to make sure that $T_{R H}^{\max }<T_{\max }$. It is reassuring to notice that in all our cases the reheating temperature is always suppressed with respect to the decompactification temperature (5.1) by inverse powers of the overall volume. Only in the case of BI with the inflaton wrapped by the visible sector, the ratio $R \equiv T_{R H}^{\max } / T_{\max } \simeq \mathcal{V}^{-1 / 4}$ is not particularly small. Hence it is important to compute the exact coefficient in from of the $\mathcal{V}^{-1 / 4}$ factor. This has already been done in [54] and the final result is:

$$
R \equiv \frac{T_{R H}^{\max }}{T_{\max }}=c \frac{(\ln \mathcal{V})^{3 / 2}}{\mathcal{V}^{1 / 4}} \text { with } c \simeq \frac{W_{0} e^{K_{c s} / 2}}{2\left\langle\tau_{2}\right\rangle^{11 / 8}},
$$

For $\mathcal{V} \simeq 10^{6-7}$, the previous ratio just depends on the $c$ coefficient: $R \simeq c$. However for natural values $W_{0} \simeq K_{c s} \simeq \mathcal{O}(1)$ and $\left\langle\tau_{2}\right\rangle \simeq \mathcal{O}(10), c \simeq 0.1-0.01$, and so we correctly have $R<1$. In the fine-tuned cases when $R$ becomes dangerously of the order one, larger values of the volume seem to be favoured. However for $\mathcal{V}>10^{7}$, the inflaton fluctuations are not able anymore to generate the right amount of density perturbations, which could be, on the other hand, produced by some non-standard mechanism, like a curvaton or a modulating field [23].

Let us now summarise the main results we have found in this paper:

- In any LVS hidden sectors are always present along with the visible sector.

- The ubiquitous presence of a hidden sector wrapping the inflaton 4-cycle implies that BI needs fine-tuning whereas FI does not.

- The hidden sector and the visible sector are not directly coupled, and furthermore the inflaton coupling to visible sector dof can never be made stronger than its coupling to hidden sector $d o f$.

- Two generic problems arise:

1. Inflaton energy dumping to hidden, instead of visible, sector dof. As such this is not a problem provided the hidden sector dof are relativistic and there exists a curvaton mechanism which is responsible for creating the perturbations and the visible sector

\footnotetext{
${ }^{14}$ Note that within the MSSM, as it is the case for many scenarios, the final reheating temperature can be as low as $1 \mathrm{TeV}$.
} 
matter. Such a scenario was considered in past [55, where the MSSM Higgs would dominate the energy density during its oscillations and would create all the visible dof. It would also be interesting to investigate if this problem could be solved by building a curvaton model along the lines of 23] where the curvaton dominates the energy density.

2. Overproduction of hidden sector dark matter if there exists a massive long lived species, otherwise there would be too many new relativistic dof, which would spoil the BBN predictions.

- A possible solution could be to render the inflaton decay to hidden sector dof kinematically forbidden. This sets severe constraints on hidden sector model building leaving as the most promising possibility just a pure $N=1$ SYM theory. This is always the case for each hidden sector at the quiver locus and in the geometric regime if the inflaton is not wrapped by the visible sector. On the contrary, in the geometric regime case with the inflaton wrapped by the visible sector, the requirement of having just a pure SYM theory for the hidden sector not wrapping the inflaton 4-cycle can be relaxed.

- This constraint seems to be in contrast with the generic presence of hidden photons whose study received a lot of attention recently [56]. More precisely, the appearance of hidden photons living on small or large cycles seems to be compatible with reheating only for models in the geometric regime with the inflaton 4-cycle wrapped by the visible sector. All the other cases seem to be incompatible with the existence of hidden photons.

Assuming that these problems can be solved by an appropriate brane construction of the hidden sector, let us summarise our results for the maximal reheating temperature of the visible sector:

\begin{tabular}{c||c|c} 
& Geometric Regime & Quiver Locus \\
\hline \hline & Inflaton not wrapped by the visible sector: & \\
Blow-up & $T_{R H}^{\max } \sim M_{P} \mathcal{V}^{-2} \sim 10^{6-8} \mathrm{GeV}$ for $\mathcal{V} \sim 10^{6-7}$ & $T_{R H}^{\max } \sim M_{P} \mathcal{V}^{-5 / 2} \sim 10^{2-4} \mathrm{GeV}$ \\
Inflation & Inflaton wrapped by the visible sector: & for $\mathcal{V} \sim 10^{6-7}$ \\
& $T_{R H}^{\max } \sim M_{P} \mathcal{V}^{-1} \sim 10^{12-13} \mathrm{GeV}$ for $\mathcal{V} \sim 10^{6-7}$ & \\
\hline \multirow{2}{*}{ Fibre } & Inflaton not wrapped by the visible sector: & \\
Inflation & $T_{R H}^{\max } \sim M_{P} \mathcal{V}^{-17 / 6} \sim 10^{6-9} \mathrm{GeV}$ for $\mathcal{V} \sim 10^{3-4}$ & $T_{R H}^{\max } \sim M_{P} \mathcal{V}^{-10 / 3} \sim 10^{4-7} \mathrm{GeV}$ \\
& Inflaton wrapped by the visible sector: & for $\mathcal{V} \sim 10^{3-4}$ \\
& $T_{R H}^{\max } \sim M_{P} \mathcal{V}^{-5 / 2} \sim 10^{8-10} \mathrm{GeV}$ for $\mathcal{V} \sim 10^{3-4}$ &
\end{tabular}

We stress that only the model of $\mathrm{BI}$ with the visible sector at the quiver locus gives rise to a viable reheating with $\mathrm{TeV}$ scale SUSY at the same time. In all other cases $M_{\text {soft }} \gg 1 \mathrm{TeV}$. Let us now assume that the inflaton is only responsible to drive inflation but the density fluctuations are generated in a non-standard way by a second curvaton or modulating field [23]. In this way we still obtain inflation but the volume could be set so to obtain TeV scale SUSY. The resulting maximal reheating temperature in the visible sector would turn out to be:

1. $\mathrm{BI}$ in the geometric regime with the inflaton not wrapped by the visible sector: for $\mathcal{V} \simeq 10^{14}$ (as needed to obtain TeV scale SUSY in the geometric regime), we would obtain $T_{R H}^{\max } \simeq 10$ $\mathrm{eV}$. This is in clear disagreement with the lower bound coming from BBN: $T_{R H}^{\max }>1 \mathrm{MeV}$. Thus we infer that this model does not seem to be compatible with TeV scale SUSY.

2. $\mathrm{BI}$ in the geometric regime with the inflaton wrapped by the visible sector: for $\mathcal{V} \simeq 10^{14}$, we would obtain $T_{R H}^{\max } \simeq 5 \cdot 10^{5} \mathrm{GeV}$ which is not in contrast with any observation. Thus we infer that this model could be made compatible with TeV scale SUSY. 
3. FI in the geometric regime with the inflaton not wrapped by the visible sector: for $\mathcal{V} \simeq 10^{14}$, and $c \simeq 5 \cdot 10^{-2}$ we would obtain $T_{R H}^{\max } \simeq 10^{-14} \mathrm{eV}$, which is unrealistically small. Thus we infer that this model does not seem to be compatible with TeV scale SUSY.

4. FI in the geometric regime with the inflaton wrapped by the visible sector: for $\mathcal{V} \simeq 10^{14}$, and $c \simeq 5 \cdot 10^{-2}$ we would obtain $T_{R H}^{\max } \simeq 10^{-8} \mathrm{eV}$, which is unrealistically small. Thus we infer that this model does not seem to be compatible with TeV scale SUSY. On top of this, we would obtain an unrealistically small value for the visible sector gauge coupling: $\alpha_{v i s}^{-1}=\left\langle\tau_{1}\right\rangle=c \mathcal{V}^{2 / 3} \simeq 10^{8}$.

5. FI at the quiver locus : for $\mathcal{V} \simeq 10^{6}$ (as needed to obtain TeV scale SUSY at the quiver locus), we would obtain $T_{R H}^{\max } \simeq 1 \mathrm{MeV}$ which is of the same order of magnitude as $T_{B B N}$. Thus we infer that this model could be made compatible with TeV scale SUSY only if $T_{R H}^{\max }$ turns out to be actually a bit higher than $T_{B B N}$ and the matter-antimatter asymmetry is generated in a non-thermal way.

Thus, even though FI does not require any fine-tuning of the coefficient of the string loop corrections, it seems very difficult to obtain a viable reheating for these models which is also compatible with TeV scale SUSY. On the contrary, both BI at the quiver locus and in the geometric regime with the inflaton wrapped by the visible sector, seems to be more promising to achieve inflation, reheating and TeV scale SUSY at the same time.

We finally stress that our estimate of the reheating temperature was based on the approximation of sudden thermalisation of the visible sector dof. In reality it is more likely that the system slowly evolves to thermal equilibrium with an actual reheating temperature $T_{R H}<T_{R H}^{\max }$. However we shall leave the detailed study of the thermalisation process for future investigation.

\section{Acknowledgments}

We would like to thank Neil Barnaby, Wildfried Buchmueller, Cliff Burgess, Joe Conlon, Manuel Drees, Mark Goodsell, Thomas Grimm, Tetsutaro Higaki, Sven Krippendorf, Oleg Lebedev, Fernando Quevedo, Gianmassimo Tasinato for helpful discussions and explanations. We are also grateful to Fernando Quevedo and Cliff Burgess for commenting on the final draft of this paper. The research of AM is partly supported by the European Union through Marie Curie Research and Training Network "UNIVERSENET" (MRTN-CT-2006-035863).

\section{A. Calculation of the moduli couplings}

In this appendix we shall derive for each case studied, the canonical normalisation which is then needed to derive the moduli mass spectrum and their couplings to visible and hidden sector dof.

\section{A.1 Blow-up Inflation}

Without loss of generality but just in order to simplify our calculations, we shall focus on compactifications on Calabi-Yau orientifolds with three instead of four Kähler moduli. The overall volume looks like:

$$
\mathcal{V}=\alpha\left(\tau_{1}^{3 / 2}-\gamma_{2} \tau_{2}^{3 / 2}-\gamma_{3} \tau_{3}^{3 / 2}\right)
$$

\section{A.1.1 Geometric regime}

Let us first of all start with the canonical normalisation and then compute the moduli couplings to ordinary and supersymmetric particles. 


\section{Canonical normalisation}

After axion minimisation, the scalar potential takes the form:

$$
V=\frac{\lambda_{2} \sqrt{\tau_{2}} e^{-2 a_{2} \tau_{2}}}{\mathcal{V}}+\frac{\lambda_{3} \sqrt{\tau_{3}} e^{-2 a_{3} \tau_{3}}}{\mathcal{V}}-\frac{\mu_{2} \tau_{2} e^{-a_{2} \tau_{2}}}{\mathcal{V}^{2}}-\frac{\mu_{3} \tau_{3} e^{-a_{3} \tau_{3}}}{\mathcal{V}^{2}}+\frac{\nu}{\mathcal{V}^{3}}
$$

where $\lambda_{i}=8 a_{i}^{2} A_{i}^{2} /\left(3 \alpha \gamma_{i}\right) \forall i=2,3, \mu_{j}=4 W_{0} a_{j} A_{j} \forall j=2,3$, and $\nu=3 \xi W_{0}^{2} /\left(4 g_{s}^{3 / 2}\right)$. The global minimum is located at:

$$
a_{i}\left\langle\tau_{i}\right\rangle=\left(\frac{4 \nu}{J}\right)^{2 / 3}, \quad\langle\mathcal{V}\rangle=\left(\frac{\mu_{i}}{2 \lambda_{i}}\right) \sqrt{\left\langle\tau_{i}\right\rangle} e^{a_{i}\left\langle\tau_{i}\right\rangle}, \quad \forall i=2,3
$$

where:

$$
J \equiv \sum_{i=2}^{3}\left(\frac{\mu_{i}^{2}}{\lambda_{i} a_{i}^{3 / 2}}\right)
$$

Let us now work out the canonical normalisation around the minimum, starting expanding each modulus around its VEV:

$$
\tau_{i}=\left\langle\tau_{i}\right\rangle+\delta \tau_{i}, \quad \forall i=1,2,3
$$

Then the Lagrangian takes the form:

$$
\mathcal{L}=K_{i j} \partial_{\mu}\left(\delta \tau_{i}\right) \partial^{\mu}\left(\delta \tau_{j}\right)-\langle V\rangle-\frac{1}{2} V_{i j} \delta \tau_{i} \delta \tau_{j}+\mathcal{O}\left(\delta \tau^{3}\right)
$$

where the direct Kähler metric at leading order looks like:

$$
\mathcal{K}=\frac{9}{8\left\langle\tau_{1}\right\rangle^{2}}\left(\begin{array}{ccc}
2 / 3 & -\gamma_{2} \epsilon_{2} & -\gamma_{3} \epsilon_{3} \\
-\gamma_{2} \epsilon_{2} & \gamma_{2} \epsilon_{2}^{-1} / 3 & \gamma_{2} \gamma_{3} \epsilon_{2} \epsilon_{3} \\
-\gamma_{3} \epsilon_{3} & \gamma_{2} \gamma_{3} \epsilon_{2} \epsilon_{3} & \gamma_{3} \epsilon_{3}^{-1} / 3
\end{array}\right)
$$

with $\epsilon_{i} \equiv \sqrt{\left\langle\tau_{i}\right\rangle /\left\langle\tau_{1}\right\rangle} \ll 1 \forall i=2,3$. Writing the original moduli $\delta \tau_{i}$ in terms of the canonically normalised fields around the minimum $\delta \phi_{i}$ as:

$$
\left(\begin{array}{l}
\delta \tau_{1} \\
\delta \tau_{2} \\
\delta \tau_{3}
\end{array}\right)=\left(\vec{v}_{1}\right) \frac{\delta \phi_{1}}{\sqrt{2}}+\left(\vec{v}_{2}\right) \frac{\delta \phi_{2}}{\sqrt{2}}+\left(\vec{v}_{3}\right) \frac{\delta \phi_{3}}{\sqrt{2}},
$$

the Lagrangian A.6. takes the canonical form:

$$
\mathcal{L}=\frac{1}{2} \sum_{i=1}^{3} \partial_{\mu}\left(\delta \phi_{i}\right) \partial^{\mu}\left(\delta \phi_{i}\right)-\langle V\rangle-\frac{m_{i}^{2}}{2} \sum_{i=1}^{3} \delta \phi_{i}^{2},
$$

only if $\overrightarrow{v_{i}}$ and $m_{i}^{2}$ are, respectively, the eigenvectors and the eigenvalues of the mass-squared matrix $\left(M^{2}\right)_{i j} \equiv \frac{1}{2}\left(K^{-1}\right)_{i k} V_{k j}$. On top of this, the eigenvectors have to be normalised as ${\overrightarrow{v_{i}}}^{T} \cdot \mathcal{K} \cdot \vec{v}_{j}=\delta_{i j}$.

The inverse Kähler metric at leading order is given by:

$$
\mathcal{K}^{-1}=4\left\langle\tau_{1}\right\rangle^{2}\left(\begin{array}{ccc}
1 / 3 & \epsilon_{2}^{2} & \epsilon_{3}^{2} \\
\epsilon_{2}^{2} & 2 \epsilon_{2} /\left(3 \gamma_{2}\right) & \epsilon_{2}^{2} \epsilon_{3}^{2} \\
\epsilon_{3}^{2} & \epsilon_{2}^{2} \epsilon_{3}^{2} & 2 \epsilon_{3} /\left(3 \gamma_{3}\right)
\end{array}\right),
$$

whereas the Hessian of the scalar potential evaluated at the global minimum (A.3), at leading order, looks like:

$$
V_{i j}=\frac{\alpha^{-3}}{\left\langle\tau_{1}\right\rangle^{13 / 2}}\left(\begin{array}{ccc}
c_{1}-c_{2}\left\langle\tau_{2}\right\rangle^{3 / 2}-c_{3}\left\langle\tau_{3}\right\rangle^{3 / 2} & -4 a_{2} c_{2}\left\langle\tau_{1}\right\rangle\left\langle\tau_{2}\right\rangle^{3 / 2} / 27 & -4 a_{3} c_{3}\left\langle\tau_{1}\right\rangle\left\langle\tau_{3}\right\rangle^{3 / 2} / 27 \\
-4 a_{2} c_{2}\left\langle\tau_{1}\right\rangle\left\langle\tau_{2}\right\rangle^{3 / 2} / 27 & 8 a_{2}^{2} c_{2}\left\langle\tau_{1}\right\rangle^{2}\left\langle\tau_{2}\right\rangle^{3 / 2} / 81 & 0 \\
-4 a_{3} c_{3}\left\langle\tau_{1}\right\rangle\left\langle\tau_{3}\right\rangle^{3 / 2} / 27 & 0 & 8 a_{3}^{2} c_{3}\left\langle\tau_{1}\right\rangle^{2}\left\langle\tau_{3}\right\rangle^{3 / 2} / 81
\end{array}\right)
$$


where:

$$
c_{1} \equiv \frac{99 \nu}{4}, \quad c_{2} \equiv \frac{81 \mu_{2}^{2}}{16 \lambda_{2}}, \quad c_{3} \equiv \frac{81 \mu_{3}^{2}}{16 \lambda_{3}} .
$$

The mass-squared matrix can be obtained multiplying (A.10) by (A.11). The leading order result is (setting without loss of generality $\gamma_{2}=\gamma_{3}=A_{2}=A_{3}=W_{0}=1$ ):

$$
\mathcal{M}^{2}=\frac{\alpha^{-3}}{\left\langle\tau_{1}\right\rangle^{9 / 2}}\left(\begin{array}{ccc}
-9\left(a_{2}\left\langle\tau_{2}\right\rangle^{5 / 2}+a_{3}\left\langle\tau_{3}\right\rangle^{5 / 2}\right)(1-7 \delta) & 6 a_{2}^{2}\left\langle\tau_{1}\right\rangle\left\langle\tau_{2}\right\rangle^{5 / 2}(1-5 \delta) & 6 a_{3}^{2}\left\langle\tau_{1}\right\rangle\left\langle\tau_{3}\right\rangle^{5 / 2}(1-5 \delta) \\
-6 a_{2} \sqrt{\left\langle\tau_{1}\right\rangle}\left\langle\tau_{2}\right\rangle^{2}(1-5 \delta) & 4 a_{2}^{2}\left\langle\tau_{1}\right\rangle^{3 / 2}\left\langle\tau_{2}\right\rangle^{2}(1-3 \delta) & 6 a_{3}^{2}\left\langle\tau_{2}\right\rangle\left\langle\tau_{3}\right\rangle^{5 / 2} \\
-6 a_{3} \sqrt{\left\langle\tau_{1}\right\rangle}\left\langle\tau_{3}\right\rangle^{2}(1-5 \delta) & 6 a_{2}^{2}\left\langle\tau_{3}\right\rangle\left\langle\tau_{2}\right\rangle^{5 / 2} & 4 a_{3}^{2}\left\langle\tau_{1}\right\rangle^{3 / 2}\left\langle\tau_{3}\right\rangle^{2}(1-3 \delta)
\end{array}\right)
$$

where $\delta \equiv 1 /\left(4 a_{2}\left\langle\tau_{2}\right\rangle\right)=1 /\left(4 a_{3}\left\langle\tau_{3}\right\rangle\right) \simeq 1 /(4 \ln \mathcal{V}) \ll 1$. The two small blow-up modes $\tau_{2}$ and $\tau_{3}$ behave in the same way and so they will have the same mass and will be heavier that the large overall volume mode $\tau_{1}: m_{2} \sim m_{3} \gg m_{1}$. Therefore we can work out the leading order volume scaling of the moduli mass spectrum noticing that:

$$
\begin{aligned}
& \operatorname{Tr}\left[\mathcal{M}^{2}\right]=m_{1}^{2}+m_{2}^{2}+m_{3}^{2} \sim m_{2}^{2} \sim m_{3}^{2} \sim \frac{a_{2}^{2}\left\langle\tau_{2}\right\rangle^{2}}{\alpha^{3}\left\langle\tau_{1}\right\rangle^{3}} \sim \frac{a_{3}^{2}\left\langle\tau_{3}\right\rangle^{2}}{\alpha^{3}\left\langle\tau_{1}\right\rangle^{3}} \sim\left(\frac{\ln \mathcal{V}}{\mathcal{V}}\right)^{2} M_{P}^{2} \\
& \frac{\operatorname{Det}\left[\mathcal{M}^{2}\right]}{\operatorname{Tr}\left[\mathcal{M}^{2}\right]^{2}} \sim \frac{m_{1}^{2} m_{2}^{2} m_{3}^{2}}{m_{2}^{2} m_{3}^{2}} \sim m_{1}^{2} \sim \frac{\left(\left\langle\tau_{2}\right\rangle^{3 / 2}+\left\langle\tau_{3}\right\rangle^{3 / 2}\right)}{a_{2}\left\langle\tau_{2}\right\rangle \alpha^{3}\left\langle\tau_{1}\right\rangle^{9 / 2}} \sim \frac{\left(\left\langle\tau_{2}\right\rangle^{3 / 2}+\left\langle\tau_{3}\right\rangle^{3 / 2}\right)}{a_{3}\left\langle\tau_{3}\right\rangle \alpha^{3}\left\langle\tau_{1}\right\rangle^{9 / 2}} \sim \frac{M_{P}^{2}}{\mathcal{V}^{3} \ln \mathcal{V}}
\end{aligned}
$$

Let us now derive the corresponding eigenvectors. For the eigenvalue $m_{1}^{2}$ we have (for $\vec{v}_{1}=$ $\left.\left(x_{1}, y_{1}, z_{1}\right)\right)$ :

$$
\mathcal{M}^{2} \vec{v}_{1}=m_{1}^{2} \vec{v}_{1} \Leftrightarrow\left\{\begin{array}{c}
x_{1} \simeq a_{s}\left\langle\tau_{1}\right\rangle\left(y_{1}+z_{1}\right) \\
y_{1}=z_{1} \\
z_{1}
\end{array},\right.
$$

where without loss of generality we have set $a_{2}=a_{3}=a_{s}$ and $\left\langle\tau_{2}\right\rangle=\left\langle\tau_{3}\right\rangle=\left\langle\tau_{s}\right\rangle$. On the other hand, for the eigenvalue $m_{2}^{2}$ we get (for $\vec{v}_{2}=\left(x_{2}, y_{2}, z_{2}\right)$ ):

$$
\mathcal{M}^{2} \vec{v}_{2}=m_{2}^{2} \vec{v}_{2} \Leftrightarrow\left\{\begin{array}{c}
x_{2} \simeq\left\langle\tau_{s}\right\rangle^{1 / 2}\left\langle\tau_{1}\right\rangle^{-1 / 2} y_{2} \\
y_{2} \\
z_{2} \simeq\left\langle\tau_{s}\right\rangle^{3 / 2}\left\langle\tau_{1}\right\rangle^{-3 / 2} y_{2} \ll y_{2}
\end{array} .\right.
$$

Finally for the eigenvalue $m_{3}^{2}$ we find (for $\vec{v}_{3}=\left(x_{3}, y_{3}, z_{3}\right)$ ):

$$
\mathcal{M}^{2} \vec{v}_{3}=m_{3}^{2} \vec{v}_{3} \Leftrightarrow\left\{\begin{array}{c}
x_{3} \simeq\left\langle\tau_{s}\right\rangle^{1 / 2}\left\langle\tau_{1}\right\rangle^{-1 / 2} z_{3} \\
y_{3} \simeq\left\langle\tau_{s}\right\rangle^{3 / 2}\left\langle\tau_{1}\right\rangle^{-3 / 2} z_{3} \ll z_{3} \\
z_{3}
\end{array}\right.
$$

The remaining components which have not been fixed yet, can be worked out by properly normalising the eigenvectors as:

$$
\left\{\begin{array}{c}
\vec{v}_{1} \cdot \mathcal{K} \cdot \vec{v}_{1}=1 \Leftrightarrow z_{1} \simeq a_{s}^{-1} \\
\vec{v}_{2} \cdot \mathcal{K} \cdot \vec{v}_{2}=1 \Leftrightarrow y_{2} \simeq\left\langle\tau_{s}\right\rangle^{1 / 4}\left\langle\tau_{1}\right\rangle^{3 / 4} \\
\vec{v}_{3} \cdot \mathcal{K} \cdot \vec{v}_{3}=1 \Leftrightarrow z_{3} \simeq\left\langle\tau_{s}\right\rangle^{1 / 4}\left\langle\tau_{1}\right\rangle^{3 / 4} .
\end{array}\right.
$$

Therefore the general form A.8 for the canonical normalisation takes the form:

$$
\left(\begin{array}{c}
\delta \tau_{1} \\
\delta \tau_{2} \\
\delta \tau_{3}
\end{array}\right)=\left(\begin{array}{c}
\left\langle\tau_{1}\right\rangle \\
a_{s}^{-1} \\
a_{s}^{-1}
\end{array}\right) \frac{\delta \phi_{1}}{\sqrt{2}}+\left(\begin{array}{c}
\left\langle\tau_{1}\right\rangle^{1 / 4}\left\langle\tau_{s}\right\rangle^{3 / 4} \\
\left\langle\tau_{1}\right\rangle^{3 / 4}\left\langle\tau_{s}\right\rangle^{1 / 4} \\
\left\langle\tau_{1}\right\rangle^{-3 / 4}\left\langle\tau_{s}\right\rangle^{7 / 4}
\end{array}\right) \frac{\delta \phi_{2}}{\sqrt{2}}+\left(\begin{array}{c}
\left\langle\tau_{1}\right\rangle^{1 / 4}\left\langle\tau_{s}\right\rangle^{3 / 4} \\
\left\langle\tau_{1}\right\rangle^{-3 / 4}\left\langle\tau_{s}\right\rangle^{7 / 4} \\
\left\langle\tau_{1}\right\rangle^{3 / 4}\left\langle\tau_{s}\right\rangle^{1 / 4}
\end{array}\right) \frac{\delta \phi_{3}}{\sqrt{2}}
$$


which in terms of factors of the overall volume scales as:

$$
\begin{aligned}
& \frac{\delta \tau_{1}}{\left\langle\tau_{1}\right\rangle} \simeq \mathcal{O}(1) \delta \phi_{1}+\mathcal{O}(\epsilon) \delta \phi_{2}+\mathcal{O}(\epsilon) \delta \phi_{3} \simeq \mathcal{O}(1) \delta \phi_{1}, \\
& \frac{\delta \tau_{2}}{\left\langle\tau_{2}\right\rangle} \simeq \mathcal{O}\left(\frac{1}{\ln \mathcal{V}}\right) \delta \phi_{1}+\mathcal{O}\left(\frac{1}{\epsilon}\right) \delta \phi_{2}+\mathcal{O}(\epsilon) \delta \phi_{3} \simeq \mathcal{O}\left(\frac{1}{\epsilon}\right) \delta \phi_{2}, \\
& \frac{\delta \tau_{3}}{\left\langle\tau_{3}\right\rangle} \simeq \mathcal{O}\left(\frac{1}{\ln \mathcal{V}}\right) \delta \phi_{1}+\mathcal{O}(\epsilon) \delta \phi_{2}+\mathcal{O}\left(\frac{1}{\epsilon}\right) \delta \phi_{3} \simeq \mathcal{O}\left(\frac{1}{\epsilon}\right) \delta \phi_{3},
\end{aligned}
$$

where $^{15}$ :

$$
\epsilon \equiv\left(\frac{\left\langle\tau_{s}\right\rangle}{\left\langle\tau_{1}\right\rangle}\right)^{3 / 4} \simeq \frac{10^{1 / 4}}{\mathcal{V}^{1 / 2}} \ll 1
$$

As we have already pointed out in the main part of the text, this result has a nice and clear geometric understanding. In fact, from A.20, we see that the overall volume mode is mostly given by $\delta \phi_{1}$ and then it mixes at subleading order with $\delta \phi_{2}$ and $\delta \phi_{3}$ in the same way since the volume 'sees' the two blow-up modes in the same way. On the contrary, from (A.21) and (A.22), we realise that each blow-up mode is mostly given by $\delta \phi_{2}$, or $\delta \phi_{3}$ respectively, then it mixes with the overall volume, and lastly there is an even more suppressed mixing with the other blow-up mode, reflecting the geometric separation between the two blow-up modes which are resolving two singularities located in different regions of the Calabi-Yau three-fold.

\section{Moduli couplings to visible sector particles}

Assuming that the visible MSSM-like sector is built via magnetised $D 7$-branes wrapping $\tau_{3},{ }^{16}$ the $4 \mathrm{D}$ effective field theory is completely specified by expanding $W, K$ and the gauge kinetic functions $f_{i}$ as power series in the matter fields [14]:

$$
\begin{aligned}
W & =W_{\text {mod }}(\varphi)+\mu(\varphi) H_{u} H_{d}+\frac{Y_{i j k}(\varphi)}{6} C^{i} C^{j} C^{k}+\ldots, \\
K & =K_{\text {mod }}(\varphi, \bar{\varphi})+\tilde{K}_{i \bar{j}}(\varphi, \bar{\varphi}) C^{i} C^{\bar{j}}+\left[Z(\varphi, \bar{\varphi}) H_{u} H_{d}+h . c .\right]+\ldots, \\
f_{i} & =\frac{T_{3}}{4 \pi}+h_{i}(F) S
\end{aligned}
$$

where $\varphi$ and the $C$ 's denote respectively all the moduli and matter fields. In addition $h_{i}(F)$ are 1-loop functions of the world-volume fluxes $F$ on different branes (the index $i$ runs over the three MSSM gauge group factors). Finally the moduli scaling of the Kähler potentials for matter fields $\tilde{K}_{i \bar{j}}(\varphi, \bar{\varphi})$ and $Z(\varphi, \bar{\varphi})$, reads 57 :

$$
\tilde{K}_{i \bar{j}}(\varphi, \bar{\varphi}) \sim \frac{\tau_{3}^{1 / 3}}{\tau_{1}} k_{i \bar{j}}(U) \text { and } Z(\varphi, \bar{\varphi}) \sim \frac{\tau_{3}^{1 / 3}}{\tau_{1}} z(U) .
$$

We notice that, due to the axionic shift symmetry of the imaginary part of the Kähler moduli, $T=\tau+i b$ with $b \rightarrow b+\epsilon$, the superpotential cannot depend on $b$. In addition, the holomorphicity of $W$, forbids also any dependence on $\tau=(T+\bar{T}) / 2$. Thus the superpotential $W$ can depend only on the complex structure moduli and the dilaton, but not on the Kähler moduli. This implies that there cannot be any dimension 4 operator involving Kähler moduli and ordinary MSSM particles. On the contrary, the Kähler moduli will couple only via higher order operators once we consider the normalised superpotential $e^{K / 2} W$ and expand the Kähler potential around the moduli VEVs.

\footnotetext{
${ }^{15}$ For $\tau_{s} \sim g_{s}^{-1} \sim \mathcal{O}(10)$ and $\tau_{1} \sim(\mathcal{V} / \alpha)^{2 / 3} \sim(10 \mathcal{V})^{2 / 3}$ since for an arbitrary Calabi-Yau $\alpha \sim \mathcal{O}(1 / 10)$.

${ }^{16}$ We consider the visible sector localised on a blow-up cycle which is fixed non-perturbatively even though this is not possible, since this does not affect the derivation of the volume scaling of the moduli canonical normalisation.
} 
The moduli couplings to ordinary and supersymmetric particles at high temperatures above the EW symmetry breaking scale, $T>M_{E W}$, where all the gauge bosons and matter fermions are still massless and no mixing of Higgsinos with gauginos into charginos and neutralinos has taken place yet, have already been derived in [52, 54] for the case of Swiss-cheese Calabi-Yau manifolds with just one small blow-up cycle. We shall now generalise those results for the case of more than one blow-up mode.

\section{- Couplings to visible gauge bosons}

The moduli couplings to the gauge bosons $g$ arise from the moduli dependence of the gauge kinetic function (A.26). Given that different values of MSSM gauge groups are obtained by turning on different world-volume fluxes at 1-loop, the coupling of $\tau_{3}$ to the gauge bosons is the same for $U(1), S U(2)$ and $S U(3)$. The relevant dimension 5 operator turns out to be:

$$
\mathcal{L} \supset \frac{\delta \tau_{3}}{4\left\langle\tau_{3}\right\rangle M_{P}} G_{\mu \nu} G^{\mu \nu}
$$

where $G_{\mu \nu}$ is the canonically normalised field strength. Now using the canonical normalisation A.22) we end up with the following couplings:

$$
\begin{gathered}
\mathcal{L}_{\delta \phi_{1} g g} \simeq \mathcal{O}\left(\frac{1}{4 \ln \mathcal{V}}\right) \frac{\delta \phi_{1}}{M_{P}} G_{\mu \nu} G^{\mu \nu}, \quad \mathcal{L}_{\delta \phi_{2} g g} \simeq \mathcal{O}\left(\frac{10^{1 / 4}}{4 \sqrt{\mathcal{V}}}\right) \frac{\delta \phi_{2}}{M_{P}} G_{\mu \nu} G^{\mu \nu}, \\
\mathcal{L}_{\delta \phi_{3} g g} \simeq \mathcal{O}\left(\frac{\sqrt{\mathcal{V}}}{4 \cdot 10^{1 / 4}}\right) \frac{\delta \phi_{3}}{M_{P}} G_{\mu \nu} G^{\mu \nu}
\end{gathered}
$$

\section{- Couplings to visible matter fermions}

The moduli couplings to an ordinary matter fermion $\psi$ can be worked out starting from its moduli-dependent kinetic and mass terms, then expanding the moduli around their VEVs, canonically normalising the $\psi$ kinetic terms, and finally substituting the canonical normalisation for the moduli. In terms of the canonically unnormalised moduli, the relevant operator is:

$$
\mathcal{L} \supset\left(\frac{1}{2\left\langle\tau_{1}\right\rangle} \frac{\delta \tau_{1}}{M_{P}}+\frac{1}{3\left\langle\tau_{3}\right\rangle} \frac{\delta \tau_{3}}{M_{P}}\right) \lambda_{c}\left\langle H_{c}\right\rangle \bar{\psi}_{c} \psi_{c}
$$

where the physical Yukawa coupling is given by: $\lambda_{c}=\lambda(U)\left\langle\tau_{3}\right\rangle^{-1 / 2}$. Notice that for $T>M_{E W}$ there is no direct modulus-fermion-fermion coupling since the Higgs VEV is still located at zero: $\left\langle H_{c}\right\rangle=0$. Therefore we have to consider the 4-particle vertex given by:

$$
\mathcal{L}_{i n t} \sim\left(\frac{1}{\left\langle\tau_{1}\right\rangle} \frac{\delta \tau_{1}}{M_{P}}-\frac{1}{3\left\langle\tau_{3}\right\rangle} \frac{\delta \tau_{3}}{M_{P}}\right) q_{c} \bar{\psi}_{c} \gamma^{\mu} A_{\mu}^{c} \psi_{c}
$$

where $q_{c} \equiv q /\left(2\left\langle\tau_{3}\right\rangle^{1 / 2}\right)$. The final moduli couplings look like:

$$
\begin{gathered}
\mathcal{L}_{\delta \phi_{1} g \bar{\psi} \psi} \sim \mathcal{O}(1) \frac{\delta \phi_{1}}{M_{P}} \bar{\psi}_{c} \gamma^{\mu} A_{\mu}^{c} \psi_{c}, \quad \mathcal{L}_{\delta \phi_{2} g \bar{\psi} \psi} \sim \mathcal{O}\left(\frac{2 \cdot 10^{1 / 4}}{3 \sqrt{\mathcal{V}}}\right) \frac{\delta \phi_{2}}{M_{P}} \bar{\psi}_{c} \gamma^{\mu} A_{\mu}^{c} \psi_{c} \\
\mathcal{L}_{\delta \phi_{3} g \bar{\psi} \psi} \sim \mathcal{O}\left(\frac{\sqrt{\mathcal{V}}}{3 \cdot 10^{1 / 4}}\right) \frac{\delta \phi_{3}}{M_{P}} \bar{\psi}_{c} \gamma^{\mu} A_{\mu}^{c} \psi_{c}
\end{gathered}
$$




\section{- Couplings to visible Higgs fields}

The form of moduli couplings to the canonically normalised Higgs field $H_{c}$ (either $H_{u p}$ or $\left.H_{\text {down }}\right)$, can similarly be worked out from the expansion of the Higgs kinetic term. The relevant dimension 5 operator looks like ${ }^{17}$ :

$$
\mathcal{L} \supset\left(\frac{\delta \tau_{1}}{\left\langle\tau_{1}\right\rangle M_{P}}-\frac{\delta \tau_{3}}{3\left\langle\tau_{3}\right\rangle M_{P}}\right) \partial_{\mu} \bar{H}_{c} \partial^{\mu} H_{c}
$$

Thus substituting in A.34 the expressions (A.20) and (A.22), the moduli couplings to Higgs fields take the form:

$$
\begin{gathered}
\mathcal{L}_{\delta \phi_{1} \bar{H}_{c} H_{c}} \sim \mathcal{O}(1) \frac{\delta \phi_{1}}{M_{P}} \partial_{\mu} \bar{H}_{c} \partial^{\mu} H_{c}, \quad \mathcal{L}_{\delta \phi_{2} \bar{H}_{c} H_{c}} \sim \mathcal{O}\left(\frac{2 \cdot 10^{1 / 4}}{3 \sqrt{\mathcal{V}}}\right) \frac{\delta \phi_{2}}{M_{P}} \partial_{\mu} \bar{H}_{c} \partial^{\mu} H_{c} \\
\mathcal{L}_{\delta \phi_{3} \bar{H}_{c} H_{c}} \sim \mathcal{O}\left(\frac{\sqrt{\mathcal{V}}}{3 \cdot 10^{1 / 4}}\right) \frac{\delta \phi_{3}}{M_{P}} \partial_{\mu} \bar{H}_{c} \partial^{\mu} H_{c} .
\end{gathered}
$$

\section{- Couplings to visible gauginos}

The moduli couplings to gauginos can again be derived by focusing on their moduli-dependent kinetic and soft mass terms. One first expands the moduli around their VEVs, then canonically normalises the gauginos and finally substitutes the expressions for the canonically normalised moduli fields. We recall that for a field theory living on a brane wrapping a generic small cycle $\tau_{s}$, the gaugino mass is proportional to the non-vanishing $F$-term of $\tau_{s}$ defined as $F^{s}=e^{K / 2} K^{s \bar{i}} D_{\bar{i}} W[58$. It is important then to realise that this $F$-term can take the form:

1. $F^{s} \simeq-2 W_{0} \frac{\tau_{s}}{\mathcal{V}} \Rightarrow$ at the minimum $\left|\left\langle F^{s}\right\rangle\right| \simeq \frac{W_{0}}{\langle\mathcal{V}\rangle}\left\langle\tau_{s}\right\rangle$ if $\tau_{s}$ is stabilised by $D$-terms or perturbative effects [13];

2. $F^{s}=-2 W_{0} \frac{\tau_{s}}{\mathcal{V}}+\left(\frac{8 a_{s} A_{s}}{3 \alpha \gamma_{s}}\right) \sqrt{\tau_{s}} e^{-a_{s} \tau_{s}} \Rightarrow$ at the minimum $\left|\left\langle F^{s}\right\rangle\right| \simeq \frac{W_{0}}{\langle\mathcal{V}\rangle}$ if $\tau_{s}$ is stabilised non-perturbatively [14].

The first case holds for the cycle $\tau_{3}$ supporting the visible sector while we will need to consider the second case for the cycle $\tau_{2}$ supporting the hidden sector. Hence we realise that $F^{2}$ and $F^{3}$ have the same volume scaling but a different dependence on the small cycle. This difference becomes crucial once the moduli are expanded around their VEVs in order to derive the moduli couplings, leading to a different volume scaling in the two cases. In the case of the visible sector, we need to start from the Lagrangian:

$$
\mathcal{L}=4 i \tau_{3} \lambda^{\dagger} \bar{\sigma}^{\mu} \partial_{\mu} \lambda+\left|F^{3}\right|(\lambda \lambda+\text { h.c. }),
$$

where $F^{3}$ takes the same form as in the first case above, and then expand the moduli around their VEVs. In terms of the canonically normalised fields $\lambda_{c}=2\left\langle\tau_{3}\right\rangle^{1 / 2} \lambda$, (A.37) becomes:

$$
\mathcal{L} \sim\left(1+\frac{\delta \tau_{3}}{\left\langle\tau_{3}\right\rangle M_{P}}\right)\left[\lambda_{c}^{\dagger} i \bar{\sigma}^{\mu} \partial_{\mu} \lambda_{c}+\frac{M_{1 / 2}^{h i d}}{2} \lambda_{c} \lambda_{c}\right]-\frac{3}{4} \frac{\delta \tau_{1}}{\left\langle\tau_{1}\right\rangle} \frac{M_{1 / 2}}{M_{P}}\left(\lambda_{c} \lambda_{c}+\text { h.c. }\right) .
$$

with $M_{1 / 2}=\left|\left\langle F^{3}\right\rangle\right| /\left(2\left\langle\tau_{3}\right\rangle\right) \simeq M_{P} / \mathcal{V}$. Only the last term in (A.38) contributes to the moduli decay rates due to the equations of motion. Hence by means of (A.20), we finally obtain the following

\footnotetext{
${ }^{17}$ Another coupling can be derived from the expansion of the SUSY breaking contribution to the Higgs mass given by $m_{H} \sim\left|F^{3}\right| / \tau_{3} \sim M_{P} / \mathcal{V}$ 14. However this coupling gives rise to a subleading contribution to the moduli decay rates and so we shall ignore it.
} 
couplings:

$$
\begin{aligned}
\mathcal{L}_{\delta \phi_{1} \lambda \lambda} & \sim \mathcal{O}\left(\frac{3}{4}\right) \frac{M_{1 / 2}}{M_{P}} \delta \phi_{1} \lambda \lambda \sim \mathcal{O}\left(\frac{3}{4 \mathcal{V}}\right) \delta \phi_{1} \lambda \lambda \\
\mathcal{L}_{\delta \phi_{i} \lambda \lambda} & \sim \mathcal{O}\left(\frac{3 \cdot 10^{1 / 4}}{4 \sqrt{\mathcal{V}}}\right) \frac{M_{1 / 2}}{M_{P}} \delta \phi_{i} \lambda \lambda \sim \mathcal{O}\left(\frac{3 \cdot 10^{1 / 4}}{4 \mathcal{V}^{3 / 2}}\right) \delta \phi_{i} \lambda \lambda, \quad \forall i=2,3 .
\end{aligned}
$$

It is interesting to notice that, contrary to the previous cases, there is no difference in the volume scaling of the coupling of $\delta \phi_{2}$ and $\delta \phi_{3}$ to $\lambda \lambda$. Thus the moduli couplings to gauginos do not reflect the geometric localisation of the visible sector on $\tau_{3}$ instead of $\tau_{2}$. The reason is that the moduli couple to gauginos only via the gaugino mass term, $M_{1 / 2} \simeq M_{P} / \mathcal{V}$, which depends only on the overall volume without reflecting the locality of the visible sector $D 7$-branes. However this locality becomes manifest if we look at the coupling of $\delta \phi_{2}$ to $\lambda \lambda$ plus another particle, like a gauge boson $g$ or the light modulus $\delta \phi_{1}$ :

$$
\mathcal{L}_{\delta \phi_{3} g \lambda \lambda} \sim \mathcal{O}\left(\frac{\sqrt{\mathcal{V}}}{10^{1 / 4}}\right) \frac{\delta \phi_{3}}{M_{P}} \lambda_{c}^{\dagger} \bar{\sigma}_{\mu} A^{\mu} \lambda_{c}, \quad \mathcal{L}_{\delta \phi_{3} \delta \phi_{1} \lambda \lambda} \sim \mathcal{O}\left(\frac{\sqrt{\mathcal{V}}}{10^{1 / 4}}\right) \frac{\delta \phi_{3}}{M_{P}} \delta \phi_{1} \lambda_{c} \lambda_{c} .
$$

These couplings will give rise to 3-body decay rates which turn out to be larger than the 2-body decay rate coming from the coupling (A.40).

\section{- Couplings to visible SUSY scalars}

The moduli couplings to squarks and sleptons collectively denoted as $\varphi$, are worked out from the expansion of the their kinetic and mass terms exactly as we did for the moduli couplings to the Higgs fields. Thus the results look like (A.35) and (A.36).

\section{- Couplings to visible Higgsinos}

The moduli couplings to Higgsinos can be derived in a way similar to the previous cases obtaining the following relevant part of the supergravity Lagrangian:

$$
\mathcal{L} \supset \frac{3}{2} \frac{\delta \tau_{1}}{\left\langle\tau_{1}\right\rangle M_{P}} m_{\tilde{H}}\left(\tilde{H}_{c} \tilde{H}_{c}+\text { h.c. }\right),
$$

with the physical Higgsino mass of the same order of the soft SUSY masses: $m_{\tilde{H}} \simeq M_{1 / 2} / 3 \simeq$ $M_{P} /(3 \mathcal{V})$ [14]. Now substituting in (A.42) the expression (A.20) for the canonical normalisation of the large modulus $\tau_{1}$, we obtain the following couplings:

$$
\begin{aligned}
& \mathcal{L}_{\delta \phi_{1} \tilde{H}_{c} \tilde{H}_{c}} \sim \mathcal{O}\left(\frac{3}{2}\right) \frac{m_{\tilde{H}}}{M_{P}} \delta \phi_{1} \tilde{H}_{c} \tilde{H}_{c} \sim \mathcal{O}\left(\frac{1}{2 \mathcal{V}}\right) \delta \phi_{1} \tilde{H}_{c} \tilde{H}_{c}, \\
& \mathcal{L}_{\delta \phi_{i} \tilde{H}_{c} \tilde{H}_{c}} \sim \mathcal{O}\left(\frac{3 \cdot 10^{1 / 4}}{2 \sqrt{\mathcal{V}}}\right) \frac{m_{\tilde{H}}}{M_{P}} \delta \phi_{i} \tilde{H}_{c} \tilde{H}_{c} \sim \mathcal{O}\left(\frac{10^{1 / 4}}{2 \mathcal{V}^{3 / 2}}\right) \delta \phi_{i} \tilde{H}_{c} \tilde{H}_{c}, \forall i=2,3 .
\end{aligned}
$$

Similarly to the case of the moduli couplings to gauginos, we find again that the moduli couplings to Higssinos do not reflect the locality of the visible sector, which, on the other hand, becomes manifest once we consider 4-particles interactions like the ones in (A.41).

\section{- Moduli self couplings}

The moduli self interactions can be obtained expanding the potential around the minimum as:

$$
V=V\left(\left\langle\tau_{i}\right\rangle\right)+\left.\frac{1}{2} \frac{\partial^{2} V}{\partial \tau_{i} \partial \tau_{j}}\right|_{\min } \delta \tau_{i} \delta \tau_{j}+\left.\frac{1}{3 !} \frac{\partial^{3} V}{\partial \tau_{i} \partial \tau_{j} \partial \tau_{k}}\right|_{\min } \delta \tau_{i} \delta \tau_{j} \delta \tau_{k}+\ldots
$$


and then focusing on the trilinear terms. After substituting the expressions A.20)-(A.22) for the moduli canonical normalisation, we end up with $(\forall i, j, k=2,3)$ :

$$
\begin{array}{ll}
\mathcal{L}_{111} & \simeq \mathcal{O}\left(\frac{M_{P}}{\mathcal{V}^{3}}\right) \delta \phi_{1}^{3}, \quad \mathcal{L}_{11 i} \simeq \mathcal{O}\left(\frac{M_{P}}{\mathcal{V}^{5 / 2}}\right) \delta \phi_{1}^{2} \delta \phi_{i}, \\
\mathcal{L}_{1 i j} & \simeq \mathcal{O}\left(\frac{M_{P}}{\mathcal{V}^{2}}\right) \delta \phi_{1} \delta \phi_{i} \delta \phi_{j}, \quad \mathcal{L}_{i j k} \simeq \mathcal{O}\left(\frac{M_{P}}{\mathcal{V}^{3 / 2}}\right) \delta \phi_{i} \delta \phi_{j} \delta \phi_{k} .
\end{array}
$$

\section{Moduli couplings to hidden sector particles}

We shall now assume that $\tau_{2}$ is wrapped by a stack of $D 7$-branes supporting a hidden $S U\left(N_{c}\right)$ gauge theory with $N_{f}<\left(N_{c}-1\right)$ fundamental flavours so that this theory undergoes gaugino condensation. The total superpotential and Kähler potential of the $4 \mathrm{D}$ effective field theory read:

$$
W_{\text {tot }}=W_{\text {mod }}+W_{v i s}+W_{\text {hid }}, \quad K_{\text {tot }}=K_{\text {mod }}+K_{v i s}+K_{\text {hid }},
$$

where $\left(W_{\text {mod }}+W_{\text {vis }}\right)$ is given by (A.24) and $\left(K_{\text {mod }}+K_{\text {vis }}\right)$ by (A.25). Denoting all the hidden matter fields as $\Phi, W_{\text {hid }}, K_{\text {hid }}$ and the hidden sector gauge kinetic functions $f_{\text {hid }}^{(i)}$ look like [57:

$$
W_{h i d}=\frac{Y_{i j k}^{h i d}(U)}{6} \Phi^{i} \Phi^{j} \Phi^{k}+\ldots, \quad K_{h i d}=\frac{\tau_{2}^{1 / 3}}{\tau_{1}} k_{i \bar{j}}^{h i d}(U) \Phi^{i} \Phi^{\bar{j}}+\ldots, \quad f_{h i d}^{(i)}=\frac{T_{2}}{4 \pi}+h_{h i d}^{(i)}(F) S .
$$

We shall now list the moduli couplings to all hidden sector dof following the same procedure used to derive the moduli couplings to visible sector particles.

\section{- Couplings to hidden gauge bosons}

The moduli couplings to hidden gauge bosons $X$ localised on $\tau_{2}$ can be worked out in a manner similar to the couplings to visible gauge bosons with the only difference that now the gauge kinetic function for the hidden sector scales as $f_{\text {hid }} \sim \tau_{2}+h_{\text {hid }}(F) s$. Therefore the relevant dimension 5 operators are:

$$
\begin{aligned}
\mathcal{L}_{\delta \phi_{1} X X} \sim \mathcal{O}\left(\frac{1}{4 \ln \mathcal{V}}\right) & \frac{\delta \phi_{1}}{M_{P}} G_{\mu \nu}^{h i d} G_{h i d}^{\mu \nu}, \quad \mathcal{L}_{\delta \phi_{2} X X} \sim \mathcal{O}\left(\frac{\sqrt{\mathcal{V}}}{4 \cdot 10^{1 / 4}}\right) \frac{\delta \phi_{2}}{M_{P}} G_{\mu \nu}^{h i d} G_{h i d}^{\mu \nu}, \\
& \mathcal{L}_{\delta \phi_{3} X X} \sim \mathcal{O}\left(\frac{10^{1 / 4}}{4 \sqrt{\mathcal{V}}}\right) \frac{\delta \phi_{3}}{M_{P}} G_{\mu \nu}^{h i d} G_{h i d}^{\mu \nu} .
\end{aligned}
$$

\section{- Couplings to hidden gauginos}

In order to derive the moduli couplings to hidden gauginos, we need to start from the Lagrangian:

$$
\mathcal{L}=4 i \tau_{2} \lambda^{\dagger} \bar{\sigma}^{\mu} \partial_{\mu} \lambda+\left|F^{2}\right|(\lambda \lambda+\text { h.c. }),
$$

where $F^{2}$ takes the same form as in the second case at page 42, and then expand the moduli around their VEVs. Given that $F^{2}$ has a different scaling than $F^{3}$, the moduli couplings to hidden gauginos do not have the same volume scaling as the expressions (A.39) and (A.40). In fact, in terms of the canonically normalised fields $\lambda_{c}=2\left\langle\tau_{2}\right\rangle^{1 / 2} \lambda$, (A.51) becomes:

$$
\mathcal{L} \sim\left(1+\frac{\delta \tau_{2}}{\left\langle\tau_{2}\right\rangle M_{P}}\right)\left[\lambda_{c}^{\dagger} i \bar{\sigma}^{\mu} \partial_{\mu} \lambda_{c}+\frac{M_{1 / 2}^{h i d}}{2} \lambda_{c} \lambda_{c}\right]+\left[\mathcal{O}\left(\frac{\ln \mathcal{V}}{M_{P}}\right) \frac{\delta \tau_{1}}{\left\langle\tau_{1}\right\rangle}+\mathcal{O}\left(\frac{(\ln \mathcal{V})^{2}}{M_{P}}\right) \frac{\delta \tau_{2}}{\left\langle\tau_{2}\right\rangle}\right] M_{1 / 2}^{h i d} \lambda_{c} \lambda_{c}
$$


with $M_{1 / 2}^{\text {hid }}=\left|\left\langle F^{2}\right\rangle\right| /\left(2\left\langle\tau_{2}\right\rangle\right) \sim M_{P} /(\mathcal{V} \ln \mathcal{V}) \sim M_{1 / 2}^{v i s} / \ln \mathcal{V}$. The moduli couplings to hidden gauginos can be read off from the last term in $\mathrm{A} .52$ ):

$$
\begin{aligned}
\mathcal{L}_{i n t} \sim\left(\frac{M_{1 / 2}^{h i d}}{M_{P}}\right)\left[\mathcal{O}(\ln \mathcal{V}) \delta \phi_{1}+\mathcal{O}\left(\frac{(\ln \mathcal{V})^{2} \mathcal{V}^{1 / 2}}{10^{1 / 4}}\right) \delta \phi_{2}+\mathcal{O}\left(\frac{10^{1 / 4}(\ln \mathcal{V})^{2}}{\mathcal{V}^{1 / 2}}\right) \delta \phi_{3}\right] \lambda_{c} \lambda_{c} \\
\Longrightarrow \mathcal{L}_{i n t} \sim \mathcal{O}\left(\frac{1}{\mathcal{V}}\right) \delta \phi_{1} \lambda_{c} \lambda_{c}+\mathcal{O}\left(\frac{\ln \mathcal{V}}{10^{1 / 4} \mathcal{V}^{1 / 2}}\right) \delta \phi_{2} \lambda_{c} \lambda_{c}+\mathcal{O}\left(\frac{10^{1 / 4} \ln \mathcal{V}}{\mathcal{V}^{3 / 2}}\right) \delta \phi_{3} \lambda_{c} \lambda_{c} .
\end{aligned}
$$

\section{- Couplings to hidden massless pion-like mesons}

Let us compute the inflaton coupling to a massless pion-like Goldstone boson. The effective Lagrangian for the canonically normalised field $\pi$ expanded around the moduli VEVs looks like:

$$
\mathcal{L} \sim \frac{1}{2} \frac{\partial_{\mu} \pi \partial^{\mu} \pi}{\left(1+\frac{\pi^{2}}{\Lambda^{2}}\right)^{2}}\left(1+\frac{\delta \tau_{2}}{3\left\langle\tau_{2}\right\rangle M_{P}}-\frac{\delta \tau_{1}}{\left\langle\tau_{1}\right\rangle M_{P}}\right)
$$

so that the interaction Lagrangian in terms of the canonically normalised moduli takes the form:

$$
\mathcal{L}_{i n t} \sim \mathcal{O}\left(\frac{1}{2 M_{P}}\right) \delta \phi_{1} \partial_{\mu} \pi \partial^{\mu} \pi+\mathcal{O}\left(\frac{\mathcal{V}^{1 / 2}}{6 \cdot 10^{1 / 4}}\right) \frac{\delta \phi_{2}}{M_{P}} \partial_{\mu} \pi \partial^{\mu} \pi+\mathcal{O}\left(\frac{10^{1 / 4}}{3 \mathcal{V}^{1 / 2}}\right) \frac{\delta \phi_{3}}{M_{P}} \partial_{\mu} \pi \partial^{\mu} \pi
$$

\section{- Couplings to hidden fermionic condensates}

The moduli couplings to hidden fermionic condensates $\psi_{\text {hid }}$ can be worked out again expanding the kinetic and mass terms. We obtain:

$$
\mathcal{L}_{\text {int }} \sim \mathcal{O}\left(\frac{1}{2 \sqrt{3} \mathcal{V}}\right) \delta \phi_{1} \bar{\psi}_{h i d} \psi_{\text {hid }}+\mathcal{O}\left(\frac{\ln \mathcal{V}}{\sqrt{3} \cdot 10^{1 / 4} \mathcal{V}^{1 / 2}}\right) \delta \phi_{2} \bar{\psi}_{h i d} \psi_{h i d}+\mathcal{O}\left(\frac{10^{1 / 4} \ln \mathcal{V}}{\sqrt{3} \mathcal{V}^{3 / 2}}\right) \delta \phi_{3} \bar{\psi}_{\text {hid }} \psi_{\text {hid }}
$$

\section{- Couplings to hidden massive scalar condensates}

The moduli couplings to hidden massive scalar condensates $\varphi_{\text {hid }}$ can be worked out again expanding the kinetic and mass terms. We obtain:

$$
\begin{aligned}
\mathcal{L}_{i n t} \sim & \mathcal{O}\left(\frac{1}{2}\right) \frac{\delta \phi_{1}}{M_{P}} \partial_{\mu} \varphi_{h i d} \partial^{\mu} \varphi_{h i d}+\mathcal{O}\left(\frac{\sqrt{\mathcal{V}}}{6 \cdot 10^{1 / 4}}\right) \frac{\delta \phi_{2}}{M_{P}} \partial_{\mu} \varphi_{h i d} \partial^{\mu} \varphi_{h i d} \\
& +\mathcal{O}\left(\frac{10^{1 / 4}}{3 \sqrt{\mathcal{V}}}\right) \frac{\delta \phi_{3}}{M_{P}} \partial_{\mu} \varphi_{h i d} \partial^{\mu} \varphi_{h i d} .
\end{aligned}
$$

\section{Moduli decay rates}

Having derived the moduli couplings to all ordinary and supersymmetric particles, let us now compute the volume scaling of all the possible decay rates.

\section{- Decays to visible gauge bosons}

The moduli decay rates to visible gauge bosons look like:

$$
\begin{aligned}
\Gamma_{\delta \phi_{1} \rightarrow g g} & \simeq\left[\frac{1}{64 \pi(\ln \mathcal{V})^{2}}\right] \frac{m_{1}^{3}}{M_{P}^{2}} \simeq\left[\frac{1}{64 \pi(\ln \mathcal{V})^{7 / 2}}\right] \frac{M_{P}}{\mathcal{V}^{9 / 2}} \simeq\left[\frac{5 \cdot 10^{-3}}{(\ln \mathcal{V})^{7 / 2}}\right] \frac{M_{P}}{\mathcal{V}^{9 / 2}} \\
\Gamma_{\delta \phi_{2} \rightarrow g g} & \simeq\left(\frac{\sqrt{10}}{64 \pi \mathcal{V}}\right) \frac{m_{2}^{3}}{M_{P}^{2}} \simeq\left[\frac{\sqrt{10}(\ln \mathcal{V})^{3}}{8 \pi}\right] \frac{M_{P}}{\mathcal{V}^{4}} \simeq 0.1(\ln \mathcal{V})^{3} \frac{M_{P}}{\mathcal{V}^{4}} \\
\Gamma_{\delta \phi_{3} \rightarrow g g} & \simeq\left(\frac{\mathcal{V}}{64 \sqrt{10} \pi}\right) \frac{m_{3}^{3}}{M_{P}^{2}} \simeq\left[\frac{(\ln \mathcal{V})^{3}}{8 \sqrt{10} \pi}\right] \frac{M_{P}}{\mathcal{V}^{2}} \simeq 0.01(\ln \mathcal{V})^{3} \frac{M_{P}}{\mathcal{V}^{2}}
\end{aligned}
$$




\section{- Decays to matter fermions}

As we have already pointed out, for temperatures above the EW symmetry breaking scale, $T>M_{E W}$, there is no direct modulus-fermion-fermion coupling and so there is no direct modulus decay to two ordinary fermions. On the other hand, we need to consider a three-body modulus decay to gauge boson-fermion-fermion obtaining ${ }^{18}$ :

$$
\Gamma_{\delta \phi_{1} \rightarrow g \bar{\psi} \psi} \simeq 0.01(\ln \mathcal{V})^{2} \Gamma_{\delta \phi_{1} \rightarrow g g}, \quad \Gamma_{\delta \phi_{i} \rightarrow g \bar{\psi} \psi} \simeq 0.01 \Gamma_{\delta \phi_{i} \rightarrow g g}, \quad \forall i=2,3 .
$$

\section{- Decays to visible Higgs fields}

Given that the SUSY breaking contribution to the Higgs mass $m_{H} \sim M_{P} \mathcal{V}^{-1}$ is higher than $m_{1} \sim M_{P} \mathcal{V}^{-3 / 2}$, the decay of the large modulus $\delta \phi_{1}$ to two Higgs fields is kinematically forbidden. On the other hand the small blow-up modes are heavier than $H$ by a $(\ln \mathcal{V})$ factor, as can be seen from (A.13): $m_{2} \sim m_{3} \sim M_{P}(\ln \mathcal{V}) / \mathcal{V}$. Hence both $\delta \phi_{2}$ and $\delta \phi_{3}$ can decay to two Higgs fields with the following decay rates:

$$
\begin{aligned}
& \Gamma_{\delta \phi_{2} \rightarrow \bar{H} H} \simeq\left(\frac{\sqrt{10}}{144 \pi \mathcal{V}}\right) \frac{m_{2}^{3}}{M_{P}^{2}} \simeq\left[\frac{\sqrt{10}(\ln \mathcal{V})^{3}}{18 \pi}\right] \frac{M_{P}}{\mathcal{V}^{4}} \simeq 0.05(\ln \mathcal{V})^{3} \frac{M_{P}}{\mathcal{V}^{4}} \\
& \Gamma_{\delta \phi_{3} \rightarrow \bar{H} H} \simeq\left(\frac{\mathcal{V}}{576 \sqrt{10} \pi}\right) \frac{m_{3}^{3}}{M_{P}^{2}} \simeq\left[\frac{(\ln \mathcal{V})^{3}}{72 \sqrt{10} \pi}\right] \frac{M_{P}}{\mathcal{V}^{2}} \simeq 0.001(\ln \mathcal{V})^{3} \frac{M_{P}}{\mathcal{V}^{2}}
\end{aligned}
$$

\section{- Decays to visible gauginos}

For the same kinematical reasons pointed out in the case of the moduli decays to Higgs fields, only the small blow-up modes can decay to visible gauginos with the following decay rates:

$$
\begin{aligned}
& \Gamma_{\delta \phi_{2} \rightarrow \lambda \lambda} \simeq\left(\frac{9 \sqrt{10}}{128 \pi \mathcal{V}^{3}}\right) m_{2} \simeq\left(\frac{9 \sqrt{10} \ln \mathcal{V}}{64 \pi \mathcal{V}^{3}}\right) \frac{M_{P}}{\mathcal{V}^{4}} \simeq 0.1(\ln \mathcal{V}) \frac{M_{P}}{\mathcal{V}^{4}} \\
& \Gamma_{\delta \phi_{3} \rightarrow g \lambda \lambda} \simeq\left(\frac{\sqrt{10} \mathcal{V}}{288 \pi^{3}}\right) \frac{m_{3}^{3}}{M_{P}^{2}} \simeq\left[\frac{\sqrt{10}(\ln \mathcal{V})^{3}}{36 \pi^{3}}\right] \frac{M_{P}}{\mathcal{V}^{2}} \simeq 10^{-3}(\ln \mathcal{V})^{3} \frac{M_{P}}{\mathcal{V}^{2}}
\end{aligned}
$$

\section{- Decays to visible SUSY scalars}

Only the heaviest moduli $\delta \phi_{2}$ and $\delta \phi_{3}$ can decay to squarks and sleptons since $m_{1} \sim M_{P} \mathcal{V}^{-3 / 2}<$ $m_{0} \sim M_{P} \mathcal{V}^{-1}$. The decay rates of the small blow-up modes to SUSY scalars scale as $\Gamma_{\delta \phi_{i} \rightarrow \bar{\varphi} \varphi} \sim$ $\Gamma_{\delta \phi_{i} \rightarrow \bar{H} H}, \forall i=2,3$.

\section{- Decays to visible Higgsinos}

For the same kinematical reasons stressed above, only the small blow-up modes can decay into two Higgsinos with decay rates that scale as:

$$
\Gamma_{\delta \phi_{2} \rightarrow \tilde{H} \tilde{H}} \simeq 0.05(\ln \mathcal{V}) \frac{M_{P}}{\mathcal{V}^{4}}, \quad \Gamma_{\delta \phi_{3} \rightarrow g \tilde{H} \tilde{H}} \simeq 10^{-4}(\ln \mathcal{V})^{3} \frac{M_{P}}{\mathcal{V}^{2}}
$$

\footnotetext{
${ }^{18}$ Notice that we could still have a two-body modulus decay to fermion-fermion via a 1-loop process. However this process is very suppressed with respect to the three-body decay since $\Gamma_{\delta \phi \rightarrow \bar{\psi} \psi}^{1-l o o p} \sim \alpha_{E M}^{2} \Gamma_{\delta \phi \rightarrow g g} \sim 10^{-4} \Gamma_{\delta \phi \rightarrow g g}$.
} 


\section{- Decays to the lightest modulus}

The only kinematically allowed moduli decays to other moduli are $\delta \phi_{i} \rightarrow \delta \phi_{1}+\delta \phi_{1}, \forall i=2,3$ with the corresponding decay rates:

$$
\Gamma_{\delta \phi_{i} \rightarrow \delta \phi_{1} \delta \phi_{1}} \simeq\left(\frac{1}{16 \pi \mathcal{V}^{5}}\right) \frac{M_{P}^{2}}{m_{i}} \simeq 10^{-2}(\ln \mathcal{V})^{-1} \frac{M_{P}}{\mathcal{V}^{4}}, \quad \forall i=2,3 .
$$

\section{- Decays to hidden gauge bosons}

The moduli decay rates to hidden gauge bosons $X$ look like:

$$
\begin{aligned}
& \Gamma_{\delta \phi_{1} \rightarrow X X} \simeq\left[\frac{5 \cdot 10^{-3}}{(\ln \mathcal{V})^{7 / 2}}\right] \frac{M_{P}}{\mathcal{V}^{9 / 2}}, \\
& \Gamma_{\delta \phi_{2} \rightarrow X X} \simeq 0.01(\ln \mathcal{V})^{3} \frac{M_{P}}{\mathcal{V}^{2}} \\
& \Gamma_{\delta \phi_{3} \rightarrow X X} \simeq 0.1(\ln \mathcal{V})^{3} \frac{M_{P}}{\mathcal{V}^{4}} .
\end{aligned}
$$

\section{- Decays to hidden gauginos}

Given that $\delta \phi_{1}$ is lighter than the hidden gauginos, only $\delta \phi_{2}$ and $\delta \phi_{3}$ can decay to these particles with corresponding decay rates:

$$
\begin{aligned}
& \Gamma_{\delta \phi_{2} \rightarrow \lambda_{h i d} \lambda_{h i d}} \simeq\left(\frac{(\ln \mathcal{V})^{2}}{8 \sqrt{10} \pi \mathcal{V}}\right) m_{2} \simeq 0.01(\ln \mathcal{V})^{3} \frac{M_{P}}{\mathcal{V}^{2}} \\
& \Gamma_{\delta \phi_{3} \rightarrow \lambda_{\text {hid }} \lambda_{h i d}} \simeq\left(\frac{\sqrt{10}(\ln \mathcal{V})^{2}}{8 \pi \mathcal{V}^{3}}\right) m_{3} \simeq 0.1(\ln \mathcal{V})^{3} \frac{M_{P}}{\mathcal{V}^{4}}
\end{aligned}
$$

\section{- Decays to hidden massless pion-like mesons}

The moduli decay rates to hidden massless pions scale as:

$$
\begin{aligned}
\Gamma_{\delta \phi_{1} \rightarrow \pi \pi} & \simeq\left(\frac{1}{256 \pi}\right) \frac{m_{1}^{3}}{M_{P}^{2}} \simeq\left[\frac{1}{256 \pi(\ln \mathcal{V})^{3 / 2}}\right] \frac{M_{P}}{\mathcal{V}^{9 / 2}} \simeq 10^{-3}(\ln \mathcal{V})^{-3 / 2} \frac{M_{P}}{\mathcal{V}^{9 / 2}} \\
\Gamma_{\delta \phi_{2} \rightarrow \pi \pi} & \simeq\left(\frac{\mathcal{V}}{2304 \sqrt{10} \pi}\right) \frac{m_{2}^{3}}{M_{P}^{2}} \simeq\left[\frac{(\ln \mathcal{V})^{3}}{288 \sqrt{10} \pi}\right] \frac{M_{P}}{\mathcal{V}^{2}} \simeq 10^{-4}(\ln \mathcal{V})^{3} \frac{M_{P}}{\mathcal{V}^{2}} \\
\Gamma_{\delta \phi_{3} \rightarrow \pi \pi} & \simeq\left(\frac{\sqrt{10}}{576 \pi \mathcal{V}}\right) \frac{m_{3}^{3}}{M_{P}^{2}} \simeq\left[\frac{\sqrt{10}(\ln \mathcal{V})^{3}}{72 \pi}\right] \frac{M_{P}}{\mathcal{V}^{4}} \simeq 0.01(\ln \mathcal{V})^{3} \frac{M_{P}}{\mathcal{V}^{4}}
\end{aligned}
$$

\section{- Decays to hidden fermionic condensates}

Only the heaviest moduli $\delta \phi_{2}$ and $\delta \phi_{3}$ can decay to hidden fermionic condensates with corresponding decay rates that scale as:

$$
\Gamma_{\delta \phi_{2} \rightarrow \bar{\psi}_{\text {hid }} \psi_{h i d}} \simeq 5 \cdot 10^{-3}(\ln \mathcal{V})^{3} \frac{M_{P}}{\mathcal{V}^{2}}, \quad \Gamma_{\delta \phi_{3} \rightarrow \bar{\psi}_{h i d} \psi_{h i d}} \simeq 5 \cdot 10^{-2}(\ln \mathcal{V})^{3} \frac{M_{P}}{\mathcal{V}^{4}}
$$

\section{- Decays to hidden massive scalar condensates}

Given that $\delta \phi_{1}$ is lighter than the hidden massive scalar condensates, only $\delta \phi_{2}$ and $\delta \phi_{3}$ can decay to these particles with corresponding decay rates:

$$
\Gamma_{\delta \phi_{2} \rightarrow \varphi_{h i d} \varphi_{h i d}} \simeq 10^{-4}(\ln \mathcal{V})^{3} \frac{M_{P}}{\mathcal{V}^{2}}, \quad \Gamma_{\delta \phi_{3} \rightarrow \varphi_{h i d} \varphi_{h i d}} \simeq 0.01(\ln \mathcal{V})^{3} \frac{M_{P}}{\mathcal{V}^{4}}
$$




\section{Summary}

- Light large modulus $\delta \phi_{1}$ : it can decay only to hidden and visible gauge bosons, massless fermions and hidden pions with the following decay widths:

$$
\begin{aligned}
& \Gamma_{\delta \phi_{1} \rightarrow g g}^{T O T} \simeq N_{G}^{v i s} \Gamma_{1} \simeq 10 \Gamma_{1}, \quad \Gamma_{\delta \phi_{1} \rightarrow g \bar{\psi} \psi}^{T O T} \simeq \frac{N_{F}}{100}(\ln \mathcal{V})^{2} \Gamma_{1} \simeq(\ln \mathcal{V})^{2} \Gamma_{1} \\
& \Gamma_{\delta \phi_{1} \rightarrow X X}^{T O T} \simeq N_{G}^{h i d} \Gamma_{1}, \quad \Gamma_{\delta \phi_{1} \rightarrow \pi \pi}^{T O T} \simeq 5 N_{f}^{2}(\ln \mathcal{V})^{2} \Gamma_{1}
\end{aligned}
$$

where:

$$
N_{F} \equiv N_{F}^{E M}+N_{F}^{N C}+2 N_{F}^{C C}+8 N_{F}^{Q C D}=138, \quad \Gamma_{1} \equiv\left(\frac{5 \cdot 10^{-3}}{(\ln \mathcal{V})^{7 / 2}}\right) \frac{M_{P}}{\mathcal{V}^{9 / 2}},
$$

and $N_{G}^{h i d}=\left(N_{c}^{2}-1\right)$ for a pure SYM theory or $N_{G}^{h i d}=\left(N_{c}-N_{f}\right)^{2}-1$ in the presence of hidden sector matter fields.

- Heavy blow-up mode $\delta \phi_{2}$ supporting the hidden sector: it decays to visible dof with decay widths:

$$
\begin{gathered}
\Gamma_{\delta \phi_{2} \rightarrow g g}^{T O T} \simeq \frac{N_{G}^{v i s}}{10} \frac{\Gamma_{0}}{\mathcal{V}^{2}} \simeq \frac{\Gamma_{0}}{\mathcal{V}^{2}}, \quad \Gamma_{\delta \phi_{2} \rightarrow g \bar{\psi} \psi}^{T O T} \simeq \frac{N_{F}}{1000} \frac{\Gamma_{0}}{\mathcal{V}^{2}} \simeq 0.1 \frac{\Gamma_{0}}{\mathcal{V}^{2}}, \quad \Gamma_{\delta \phi_{2} \rightarrow \bar{H} H}^{T O T} \simeq 0.1 \frac{\Gamma_{0}}{\mathcal{V}^{2}} \\
\Gamma_{\delta \phi_{2} \rightarrow \lambda \lambda}^{T O T} \simeq \frac{1}{(\ln \mathcal{V})^{2}} \frac{\Gamma_{0}}{\mathcal{V}^{2}}, \quad \Gamma_{\delta \phi_{2} \rightarrow \bar{\varphi} \varphi}^{T O T} \simeq \frac{\Gamma_{0}}{\mathcal{V}^{2}}, \quad \Gamma_{\delta \phi_{2} \rightarrow \tilde{H} \tilde{H}}^{T O T} \simeq \frac{0.1}{(\ln \mathcal{V})^{2}} \frac{\Gamma_{0}}{\mathcal{V}^{2}}
\end{gathered}
$$

whereas the decay rates to hidden sector dof look like:

$$
\begin{gathered}
\Gamma_{\delta \phi_{2} \rightarrow X X}^{T O T} \simeq \frac{N_{G}^{h i d}}{100} \Gamma_{0}, \quad \Gamma_{\delta \phi_{2} \rightarrow \lambda_{h i d} \lambda_{h i d}}^{T O T} \simeq \frac{N_{G}^{h i d}}{100} \Gamma_{0}, \quad \Gamma_{\delta \phi_{2} \rightarrow \delta \phi_{1} \delta \phi_{1}}^{T O T} \simeq \frac{10^{-2}}{(\ln \mathcal{V})^{4}} \frac{\Gamma_{0}}{\mathcal{V}^{2}} \\
\Gamma_{\delta \phi_{2} \rightarrow \pi \pi}^{T O T} \simeq 10^{-4} N_{f}^{2} \Gamma_{0}, \quad \Gamma_{\delta \phi_{2} \rightarrow \bar{\psi}_{h i d} \psi_{h i d}}^{T O T} \simeq 5 \cdot 10^{-3} N_{f}^{2} \Gamma_{0}, \quad \Gamma_{\delta \phi_{2} \rightarrow \varphi_{h i d} \varphi_{h i d}}^{T O T} \simeq 10^{-4} N_{f}^{2} \Gamma_{0},
\end{gathered}
$$

where:

$$
\Gamma_{0} \equiv(\ln \mathcal{V})^{3} \frac{M_{P}}{\mathcal{V}^{2}}
$$

- Heavy blow-up mode $\delta \phi_{3}$ supporting the visible sector: it decays to visible dof with decay widths:

$$
\begin{aligned}
\Gamma_{\delta \phi_{3} \rightarrow g g}^{T O T} \simeq \frac{N_{G}^{v i s}}{100} \Gamma_{0} \simeq 0.1 \Gamma_{0}, \quad \Gamma_{\delta \phi_{3} \rightarrow g \bar{\psi} \psi}^{T O T} \simeq 10^{-4} N_{F} \Gamma_{0} \simeq 0.01 \Gamma_{0}, \quad \Gamma_{\delta \phi_{3} \rightarrow \bar{H} H}^{T O T} \simeq 5 \cdot 10^{-3} \Gamma_{0}, \\
\Gamma_{\delta \phi_{3} \rightarrow g \lambda \lambda}^{T O T} \simeq 0.01 \Gamma_{0}, \quad \Gamma_{\delta \phi_{3} \rightarrow \bar{\varphi} \varphi}^{T O T} \simeq 0.05 \Gamma_{0}, \quad \Gamma_{\delta \phi_{3} \rightarrow g \tilde{H} \tilde{H}}^{T O T} \simeq 5 \cdot 10^{-3} \Gamma_{0},
\end{aligned}
$$

whereas the decay rates to hidden sector dof look like:

$$
\begin{aligned}
& \Gamma_{\delta \phi_{3} \rightarrow X X}^{T O T} \simeq 0.1 N_{G}^{h i d} \frac{\Gamma_{0}}{\mathcal{V}^{2}}, \quad \Gamma_{\delta \phi_{2} \rightarrow \lambda_{h i d} \lambda_{h i d}}^{T O T} \simeq 0.1 N_{G}^{h i d} \frac{\Gamma_{0}}{\mathcal{V}^{2}}, \quad \Gamma_{\delta \phi_{3} \rightarrow \delta \phi_{1} \delta \phi_{1}}^{T O T} \simeq \frac{10^{-2}}{(\ln \mathcal{V})^{4}} \frac{\Gamma_{0}}{\mathcal{V}^{2}} \\
& \Gamma_{\delta \phi_{3} \rightarrow \pi \pi}^{T O T} \simeq 0.01 N_{f}^{2} \frac{\Gamma_{0}}{\mathcal{V}^{2}}, \quad \Gamma_{\delta \phi_{3} \rightarrow \bar{\psi}_{h i d} \psi_{h i d}}^{T O T} \simeq 0.05 N_{f}^{2} \frac{\Gamma_{0}}{\mathcal{V}^{2}}, \quad \Gamma_{\delta \phi_{3} \rightarrow \varphi_{h i d} \varphi_{h i d}}^{T O T} \simeq 0.01 N_{f}^{2} \frac{\Gamma_{0}}{\mathcal{V}^{2}}
\end{aligned}
$$

We notice that, except for $\delta \phi_{1}$, the strongest moduli decay rates are to visible or hidden gauge bosons and so, from now on, we shall focus only on those couplings. 


\section{A.1.2 Quiver locus}

Let us now focus on the case when the cycle supporting the visible sector shrinks at zero size: $\tau_{3} \rightarrow 0$. We shall first of all start with the canonical normalisation and then compute the moduli couplings.

\section{Canonical normalisation}

Let us assume that the modulus $\tau_{3}$ shrinks at zero size. This can be achieved by using $D$-terms which generically take the form:

$$
V_{D} \sim g^{2}\left(\sum_{i} q_{i} \frac{\partial K}{\partial \varphi_{i}} \varphi_{i}-\xi_{3}\right)^{2},
$$

where:

$$
\xi_{3} \sim \frac{1}{\mathcal{V}} \int_{C Y} J \wedge\left(F_{3}-B\right) \wedge D_{3}
$$

is the Fayet-Iliopoulos term associated with the turning on of the gauge flux $F_{3}$ on $\tau_{3}$ (we denote with $D_{3}$ the 2 -form dual to the 4 -cycle whose volume is given by $\tau_{3}$ ). If $\tau_{3}$ is a blow-up mode which does not intersect with any other cycle, then $\xi_{3} \sim \sqrt{\tau_{3}} / \mathcal{V}$.

Given that in a large volume expansion $V_{D} \sim \mathcal{V}^{-2}$ whereas $V_{F} \sim \mathcal{V}^{-3}$, a non-vanishing $V_{D}$ would give a run-away behaviour for the volume mode. Hence we need to impose $V_{D}=0$. If the sum in (A.80) comprises also a SM singlet $\varphi_{s}$, then this requirement fixes, $\left|\varphi_{s}\right| \sim \sqrt{\xi_{3}}$, and then $\tau_{3}$ can be fixed by string loop corrections as pointed out in [13]. However if in (A.80) there are no SM singlets, then $V_{D}=0$ implies $\xi_{3}=0$ forcing $\tau_{3}$ to shrink to zero size [15.

At the quiver locus the tree-level gauge kinetic function is given by the axio-dilaton $S$ and not by $\tau_{3}$, hence in order to work out the coupling of the inflaton to visible sector $d o f$, one has to include also $S$ in the canonical normalisation. More precisely, the complete description of the effective field theory at the quiver locus is obtained by expanding around the singularity [15]:

$$
\begin{aligned}
K & \sim-2 \ln \left[\alpha\left(\tau_{1}^{3 / 2}-\gamma_{2} \tau_{2}^{3 / 2}\right)+\frac{s^{3 / 2} \xi}{2}\right]+\lambda \frac{\tau_{3}^{2}}{\mathcal{V}^{\prime}}-\ln (2 s), \\
W & \sim W_{0}+A_{2} e^{-a_{2} T_{2}}, \\
f & \sim S+h T_{3},
\end{aligned}
$$

where $s=\operatorname{Re}(S), h$ is a 1-loop factor and $\mathcal{V}^{\prime}=\alpha\left(\tau_{1}^{3 / 2}-\gamma_{2} \tau_{2}^{3 / 2}\right)$. The real part of the axiodilaton gives the string coupling $\langle s\rangle=g_{s}^{-1}$ and it is flux-stabilised at tree-level so that we are in the perturbative regime: $\langle s\rangle \sim \mathcal{O}(10)$. The direct Kähler metric at leading order and around the minimum $\left\langle\tau_{3}\right\rangle=0$ looks like:

$$
\mathcal{K}=\frac{9}{8\left\langle\tau_{1}\right\rangle^{2}}\left(\begin{array}{cccc}
2 / 3 & -\gamma_{2} \epsilon_{2} & 0 & \xi \epsilon_{s} /(2 \alpha) \\
-\gamma_{2} \epsilon_{2} & \gamma_{2} \epsilon_{2}^{-1} / 3 & 0 & -\gamma_{2} \xi \epsilon_{2} \epsilon_{s} /(2 \alpha) \\
0 & 0 & 4 \lambda \sqrt{\left\langle\tau_{1}\right\rangle} /(9 \alpha) & 0 \\
\xi \epsilon_{s} /(2 \alpha) & -\gamma_{2} \xi \epsilon_{2} \epsilon_{s} /(2 \alpha) & 0 & 2 \epsilon_{s}^{-4} / 9
\end{array}\right)
$$

with $\epsilon_{2}=\sqrt{\left\langle\tau_{2}\right\rangle /\left\langle\tau_{1}\right\rangle} \ll 1$ and $\epsilon_{s}=\sqrt{\langle s\rangle /\left\langle\tau_{1}\right\rangle} \ll 1$. It is easy to realise that at leading order, $\mathcal{K}$ is diagonal and in addition $\tau_{3}$ does not mix with any other modulus. In fact the leading order kinetic Lagrangian reads:

$$
\begin{aligned}
\mathcal{L}_{\text {kin }}^{\text {lead }}= & \frac{3}{4\left\langle\tau_{1}\right\rangle^{2}} \partial_{\mu}\left(\delta \tau_{1}\right) \partial^{\mu}\left(\delta \tau_{1}\right)+\frac{3 \gamma_{2}}{8\left\langle\tau_{1}\right\rangle^{3 / 2} \sqrt{\left\langle\tau_{2}\right\rangle}} \partial_{\mu}\left(\delta \tau_{2}\right) \partial^{\mu}\left(\delta \tau_{2}\right) \\
& +\frac{\lambda}{2 \alpha\left\langle\tau_{1}\right\rangle^{3 / 2}} \partial_{\mu}\left(\delta \tau_{3}\right) \partial^{\mu}\left(\delta \tau_{3}\right)+\frac{1}{4\langle s\rangle^{2}} \partial_{\mu}(\delta s) \partial^{\mu}(\delta s),
\end{aligned}
$$


while the subleading bit is given by:

$$
\mathcal{L}_{\text {kin }}^{\text {sublead }}=-\frac{9 \gamma_{2} \sqrt{\left\langle\tau_{2}\right\rangle}}{4\left\langle\tau_{1}\right\rangle^{5 / 2}} \partial_{\mu}\left(\delta \tau_{1}\right) \partial^{\mu}\left(\delta \tau_{2}\right)+\frac{9 \xi \sqrt{\langle s\rangle}}{8 \alpha\left\langle\tau_{1}\right\rangle^{5 / 2}} \partial_{\mu}\left(\delta \tau_{1}\right) \partial^{\mu}(\delta s)-\frac{9 \gamma_{2} \xi \sqrt{\langle s\rangle} \sqrt{\left\langle\tau_{2}\right\rangle}}{8 \alpha\left\langle\tau_{1}\right\rangle^{3}} \partial_{\mu}\left(\delta \tau_{2}\right) \partial^{\mu}(\delta s) .
$$

The kinetic Lagrangian A.86 can be put in the canonical form defining:

$$
\delta \tau_{1}=\sqrt{\frac{2}{3}}\left\langle\tau_{1}\right\rangle \delta \phi_{1}, \quad \delta \tau_{2}=\sqrt{\frac{4}{3 \gamma_{2}}}\left\langle\tau_{1}\right\rangle^{3 / 4}\left\langle\tau_{2}\right\rangle^{1 / 4} \delta \phi_{2}, \quad \delta \tau_{3}=\sqrt{\frac{\alpha}{\lambda}}\left\langle\tau_{1}\right\rangle^{3 / 4} \delta \phi_{3}, \quad \delta s=\sqrt{2}\langle s\rangle \delta \phi_{s} .
$$

However the Lagrangian A.87 introduces a subleading mixing between $\delta \tau_{1}, \delta \tau_{2}$ and $\delta s$ whose volume scaling can be derived by imposing to have a vanishing $\mathcal{L}_{\text {kin }}^{\text {sublead }}$. We can start writing:

$$
\begin{aligned}
\delta \tau_{1} & =\sqrt{\frac{2}{3}}\left\langle\tau_{1}\right\rangle \delta \phi_{1}+k_{12}\left\langle\tau_{1}\right\rangle^{c_{12}} \delta \phi_{2}+k_{1 s}\left\langle\tau_{1}\right\rangle^{c_{1 s}} \delta \phi_{s}, \\
\delta \tau_{2} & =k_{21}\left\langle\tau_{1}\right\rangle^{c_{21}} \delta \phi_{1}+\sqrt{\frac{4}{3 \gamma_{2}}}\left\langle\tau_{1}\right\rangle^{3 / 4}\left\langle\tau_{2}\right\rangle^{1 / 4} \delta \phi_{2}+k_{2 s}\left\langle\tau_{1}\right\rangle^{c_{2 s}} \delta \phi_{s}, \\
\delta \tau_{3} & =\sqrt{\frac{\alpha}{\lambda}}\left\langle\tau_{1}\right\rangle^{3 / 4} \delta \phi_{3}, \\
\delta s & =k_{s 1}\left\langle\tau_{1}\right\rangle^{c_{s 1}} \delta \phi_{1}+k_{s 2}\left\langle\tau_{1}\right\rangle^{c_{s 2}} \delta \phi_{2}+\sqrt{2}\langle s\rangle \delta \phi_{s},
\end{aligned}
$$

where the coefficients $k_{i j}, i \neq j, i, j=1,2, s$, can involve only powers of the small moduli $\tau_{2}$ and $s$ while the $c$-coefficients have to satisfy the following constraints:

$$
c_{12}=c_{1 s}<1, \quad c_{2 s}<c_{21}<3 / 4, \quad c_{s 2}<c_{s 1}<0
$$

reflecting the fact that in (A.87) the mixing of $\delta \tau_{1}$ with $\delta \tau_{2}$ and $\delta s$ takes places at the same order in a large volume expansion whereas the mixing between $\delta \tau_{2}$ and $\delta s$ occurs only at subleading order. Now substituting the general form for the canonical normalisation A.89)-A.92 in (A.86) and (A.87), and then requiring a vanishing mixing between the moduli at leading order in a large volume expansion, we obtain the following results:

$$
c_{12}=c_{1 s}=1 / 4, \quad c_{21}=0, \quad c_{2 s}=-3 / 4, \quad c_{s 1}=-3 / 4, \quad c_{s 2}=-3 / 2,
$$

which are clearly in agreement with the constraints (A.93). Writing now the volume as $\mathcal{V} \simeq \tau_{1}^{3 / 2}$, the expressions A.89)- A.92 take the final form:

$$
\begin{aligned}
\delta \tau_{1} & \sim \mathcal{O}\left(\mathcal{V}^{2 / 3}\right) \delta \phi_{1}+\mathcal{O}\left(\mathcal{V}^{1 / 6}\right) \delta \phi_{2}+\mathcal{O}\left(\mathcal{V}^{1 / 6}\right) \delta \phi_{s} \sim \mathcal{O}\left(\mathcal{V}^{2 / 3}\right) \delta \phi_{1} \\
\delta \tau_{2} & \sim \mathcal{O}(1) \delta \phi_{1}+\mathcal{O}\left(\mathcal{V}^{1 / 2}\right) \delta \phi_{2}+\mathcal{O}\left(\mathcal{V}^{-1 / 2}\right) \delta \phi_{s} \sim \mathcal{O}\left(\mathcal{V}^{1 / 2}\right) \delta \phi_{2}, \\
\delta \tau_{3} & \sim \mathcal{O}\left(\mathcal{V}^{1 / 2}\right) \delta \phi_{3}, \\
\delta s & \sim \mathcal{O}\left(\mathcal{V}^{-1 / 2}\right) \delta \phi_{1}+\mathcal{O}\left(\mathcal{V}^{-1}\right) \delta \phi_{2}+\mathcal{O}(1) \delta \phi_{s} \sim \mathcal{O}(1) \delta \phi_{s}
\end{aligned}
$$

\section{Moduli couplings}

The couplings of $\delta \phi_{1}, \delta \phi_{2}$ and $\delta \phi_{s}$ to visible gauge bosons can be obtained from the tree-level gauge kinetic function $\operatorname{Re}(f)_{\text {tree }}=s$ while the coupling of $\delta \phi_{3}$ to visible gauge bosons can be derived from the 1-loop gauge kinetic function $\operatorname{Re}(f)_{1-\text { loop }}=h \tau_{3}$. In fact the kinetic terms read:

$$
\mathcal{L}_{\text {gauge }}=-\frac{\left(s+h \tau_{3}\right)}{M_{P}} F_{\mu \nu} F^{\mu \nu} .
$$


We then expand $s$ and $\tau_{3}$ around their VEVs and go to the canonically normalised field strength $G_{\mu \nu}$ defined as:

$$
G_{\mu \nu}=2 \sqrt{\langle s\rangle} F_{\mu \nu}
$$

and obtain:

$$
\mathcal{L}_{\text {gauge }}=-\frac{1}{4} G_{\mu \nu} G^{\mu \nu}-\frac{\delta s}{4 M_{P}\langle s\rangle} G_{\mu \nu} G^{\mu \nu}-\frac{h \delta \tau_{3}}{4 M_{P}\langle s\rangle} G_{\mu \nu} G^{\mu \nu} .
$$

Now by substituting in (A.101) the expressions (A.98) for $\delta s$ and (A.97) for $\delta \tau_{3}$, we obtain the moduli couplings to the visible gauge bosons. It turns out that $\delta \phi_{3}$ couples as $1 / M_{s}, \delta \phi_{s}$ as $1 / M_{P}$, $\delta \phi_{1}$ as $1 /\left(M_{P} \sqrt{\mathcal{V}}\right)$, and $\delta \phi_{2}$ as $1 /\left(M_{P} \mathcal{V}\right)$. Hence the coupling of $\delta \phi_{2}$ to visible sector gauge bosons is even more suppressed than in the geometric case. This result is not surprising since it is reflecting the presence of a singularity besides the geometrical separation between the two blow-up cycles.

We finally stress that the cycle $\tau_{2}$ supporting the hidden sector responsible for the generation of the non-perturbative superpotential, is fixed at a size larger than the string scale. Thus the moduli couplings to hidden sector particles take the same form as in section A.1.1.

\section{A.2 Fibre Inflation}

In the case of FI, the overall volume reads:

$$
\mathcal{V}=\alpha\left(\sqrt{\tau_{1}} \tau_{2}-\sum_{i} \gamma_{i} \tau_{i}^{3 / 2}\right),
$$

and so the only difference with the case of Swiss-cheese Calabi-Yau manifolds used for BI is that the overall volume can be approximated as $\mathcal{V} \simeq \sqrt{\tau_{1}} \tau_{2}$ instead of just $\mathcal{V} \simeq \tau_{1}^{3 / 2}$. In addition we have seen that, in this case, both $\tau_{1}$ and $\tau_{2}$ are large 4-cycles stabilised at subleading 1-loop order.

This implies that the same considerations of the previous sections for the moduli canonical normalisation and couplings apply also in this case where we have just to work out the 'finestructure' mixing between $\tau_{1}$ and $\tau_{2}$. In fact, the same structure of the overall volume implies that we shall obtain the same mixing between large and small moduli.

\section{A.2.1 Canonical normalisation without loop corrections}

Let us derive the mixing between the two large moduli $\tau_{1}$ and $\tau_{2}$ once all the blow-up modes have been fixed at their VEVs: $a_{i}\left\langle\tau_{i}\right\rangle \sim \ln \mathcal{V}$. We shall start by neglecting the loop corrections. Recalling the definition of $J$ from (2.10), the scalar potential schematically looks like:

$$
V \simeq \frac{-J(\ln \mathcal{V})^{3 / 2}+\hat{\xi}}{\mathcal{V}^{3}}=J\left[\frac{-(\ln \mathcal{V})^{3 / 2}+\tilde{\xi}}{\mathcal{V}^{3}}\right],
$$

where $\tilde{\xi} \equiv \hat{\xi} / J, \mathcal{V} \simeq \sqrt{\tau_{1}} \tau_{2}$ and the inverse Kähler metric is given by:

$$
K_{i j}^{-1}=4\left(\begin{array}{cc}
\left\langle\tau_{1}\right\rangle^{2} & \frac{\sqrt{\left\langle\tau_{1}\right\rangle}}{2}(\ln \mathcal{V})^{3 / 2} \\
\frac{\sqrt{\left\langle\tau_{1}\right\rangle}}{2}(\ln \mathcal{V})^{3 / 2} & \left\langle\tau_{2}\right\rangle^{2} / 2
\end{array}\right) .
$$

The presence of a flat direction can be inferred by the fact that the first derivative of the scalar potential with respect to $\tau_{1}, V_{1}$, is proportional to the derivative with respect to $\tau_{2}, V_{2}$ :

$$
V_{1}=\left(\frac{\tau_{2}}{2 \tau_{1}}\right) V_{2}
$$

therefore the vanishing of $V_{2}$ automatically implies also the vanishing of $V_{1}$. The only direction which is fixed is the one corresponding to the overall volume: $\mathcal{V} \simeq \exp \left(\tilde{\xi}^{2 / 3}\right)$. 
In order to diagonalise simultaneously both the kinetic terms and the mass matrix around the minimum, one has to find the eigenvalues and eigenvectors of the following matrix:

$M_{i k}^{2}=\left.K_{i j}^{-1} V_{j k}\right|_{\mathcal{V}=\exp \left(\tilde{\xi}^{2 / 3}\right)}=\frac{9}{2} \frac{\sqrt{\ln \mathcal{V}}}{\mathcal{V}^{3}}\left(\begin{array}{cc}1+\epsilon & \frac{2\left\langle\tau_{1}\right\rangle}{\left\langle\tau_{2}\right\rangle}(1+\epsilon) \\ \frac{\left\langle\tau_{2}\right\rangle}{\left\langle\tau_{1}\right\rangle}(1+\epsilon) & 2+\epsilon\end{array}\right), \quad$ with $\epsilon=\mathcal{O}\left(\frac{(\ln \mathcal{V})^{3 / 2}}{\mathcal{V}}\right) \ll 1$,

where this matrix has been evaluated at:

$$
\tilde{\xi}=\frac{1}{2}\left[2(\ln \mathcal{V})^{3 / 2}-\sqrt{\ln \mathcal{V}}\right] \simeq(\ln \mathcal{V})^{3 / 2}
$$

which is the solution of $V_{1} \propto V_{2}=0$. The eigenvalues with the corresponding eigenvectors turn out to be:

$$
\begin{array}{lll}
m_{1}^{2}=0 & \longleftrightarrow & \vec{v}_{1}=\left(\begin{array}{c}
-\frac{2\left\langle\tau_{1}\right\rangle}{\left\langle\tau_{2}\right\rangle} x \\
x
\end{array}\right), \\
m_{2}^{2} \simeq J \frac{\sqrt{\ln \mathcal{V}}}{\mathcal{V}^{3}} \quad \longleftrightarrow \quad \vec{v}_{2}=\left(\begin{array}{c}
\frac{\left\langle\tau_{1}\right\rangle}{\left\langle\tau_{2}\right\rangle} y(1+\epsilon) \\
y
\end{array}\right),
\end{array}
$$

and so the canonical normalisation around the minimum looks like:

$$
\left(\begin{array}{c}
\delta \tau_{1} \\
\delta \tau_{2}
\end{array}\right)=\vec{v}_{1} \frac{\delta \phi_{1}}{\sqrt{2}}+\vec{v}_{2} \frac{\delta \phi_{2}}{\sqrt{2}}=\left(\begin{array}{c}
-\frac{2\left\langle\tau_{1}\right\rangle}{\left\langle\tau_{2}\right\rangle} \\
x
\end{array}\right) \frac{\delta \phi_{1}}{\sqrt{2}}+\left(\begin{array}{c}
\frac{\left\langle\tau_{1}\right\rangle}{\left\langle\tau_{2}\right\rangle} y(1+\epsilon) \\
y
\end{array}\right) \frac{\delta \phi_{2}}{\sqrt{2}} .
$$

Notice that from (2.10):

$$
J \simeq \frac{\hat{\xi}}{\left(a_{i}\left\langle\tau_{i}\right\rangle\right)^{3 / 2}} \simeq \frac{\mathcal{O}(1)}{\left(g_{s} \ln \mathcal{V}\right)^{3 / 2}}
$$

and so the non-vanishing mass-squared (A.108) scales exactly as A.14):

$$
m_{2}^{2} \simeq \frac{\mathcal{O}(1)}{g_{s}^{3 / 2} \mathcal{V}^{3} \ln \mathcal{V}}
$$

In order to work out the values of $x$ and $y$, we need to impose $\vec{v}_{\alpha}^{T} \cdot \mathcal{K} \cdot \vec{v}_{\beta}=\delta_{\alpha \beta}$ which implies $x=y / \sqrt{2}=2\left\langle\tau_{2}\right\rangle(1+\epsilon) / \sqrt{6}$. Therefore the final canonical normalisation looks like:

$$
\left\{\begin{array}{c}
\frac{\delta \tau_{1}}{\left\langle\tau_{1}\right\rangle}=-\left[\frac{2}{\sqrt{3}}(1+\epsilon)\right] \delta \phi_{1}+\left[\sqrt{\frac{2}{3}}(1+\epsilon)\right] \delta \phi_{2} \\
\frac{\delta \tau_{2}}{\left\langle\tau_{2}\right\rangle}=\left[\frac{1}{\sqrt{3}}(1+\epsilon)\right] \delta \phi_{1}+\left[\sqrt{\frac{2}{3}}(1+\epsilon)\right] \delta \phi_{2}
\end{array}\right.
$$

The physical interpretation of these two mass-eigenstates becomes clearer once we notice that from $\mathcal{V} \simeq \sqrt{\tau_{1}} \tau_{2}$, we obtain:

$$
\frac{1}{\sqrt{3}} \frac{\delta \mathcal{V}}{\mathcal{V}}=\frac{1}{2 \sqrt{3}} \frac{\delta \tau_{1}}{\left\langle\tau_{1}\right\rangle}+\frac{1}{\sqrt{3}} \frac{\delta \tau_{2}}{\left\langle\tau_{2}\right\rangle}=\delta \phi_{2}(1+\epsilon)
$$

Hence $\delta \phi_{2}$ plays the rôle of the overall volume. This is the reason why this mass-eigenstate has a mass-squared of the order $\mathcal{V}^{-3}$ given that the volume is stabilised at that order. On the other hand, $\delta \phi_{1}$ cannot be expressed as a function of just the overall volume, since:

$$
\delta \phi_{1}(1+\epsilon)=\frac{1}{\sqrt{3}} \frac{\delta \mathcal{V}}{\mathcal{V}}-\frac{\sqrt{3}}{2} \frac{\delta \tau_{1}}{\left\langle\tau_{1}\right\rangle}
$$

Due to the dependence of $\delta \phi_{1}$ on $\delta \tau_{1}$ and the fact that, without loop corrections, $\tau_{1}$ is a flat direction, the mass-eigenstate $\delta \phi_{1}$ correctly turns out to be massless. 
On top of this, at this level of analysis, besides being still massless, $\delta \phi_{1}$ does not couple to any gauge boson living on a stack of $D 7$-branes wrapping any of the small blow-up cycles. This is because, at this stage, $\delta \phi_{1}$ does not mix with any blow-up mode. In order to see this important implication, let us now expand the blow-up modes around their VEVs. The leading order kinetic Lagrangian then looks like (setting without loss of generality $\alpha=\gamma_{i}=1 \forall i$ ):

$$
\begin{aligned}
-\mathcal{L}_{k i n} & =\frac{\partial^{\mu}\left(\delta \tau_{1}\right) \partial_{\mu}\left(\delta \tau_{1}\right)}{4\left\langle\tau_{1}\right\rangle^{2}}+\frac{\partial^{\mu}\left(\delta \tau_{2}\right) \partial_{\mu}\left(\delta \tau_{2}\right)}{2\left\langle\tau_{2}\right\rangle^{2}}+\frac{3}{8} \sum_{i} \frac{\partial^{\mu}\left(\delta \tau_{i}\right) \partial_{\mu}\left(\delta \tau_{i}\right)}{\mathcal{V} \sqrt{\left\langle\tau_{i}\right\rangle}}+\frac{\sum_{i}\left\langle\tau_{i}\right\rangle^{3 / 2}}{2 \mathcal{V}} \frac{\partial^{\mu}\left(\delta \tau_{1}\right)}{\left\langle\tau_{1}\right\rangle} \frac{\partial_{\mu}\left(\delta \tau_{2}\right)}{\left\langle\tau_{2}\right\rangle} \\
& -\frac{3}{2} \sum_{i} \frac{\sqrt{\left\langle\tau_{i}\right\rangle}}{\mathcal{V}}\left(\frac{1}{2} \frac{\partial^{\mu}\left(\delta \tau_{1}\right)}{\left\langle\tau_{1}\right\rangle}+\frac{\partial^{\mu}\left(\delta \tau_{2}\right)}{\left\langle\tau_{2}\right\rangle}\right) \partial_{\mu}\left(\delta \tau_{i}\right)+\frac{9}{4} \sum_{j>i} \frac{\sqrt{\left\langle\tau_{i}\right\rangle\left\langle\tau_{j}\right\rangle}}{\mathcal{V}^{2}} \partial^{\mu}\left(\delta \tau_{i}\right) \partial_{\mu}\left(\delta \tau_{j}\right), \quad \text { (A.115) }
\end{aligned}
$$

which in terms of the canonical normalisation (A.112) takes the form:

$$
\begin{gathered}
-\mathcal{L}_{k i n}=\frac{1}{2} \partial_{\mu}\left(\delta \phi_{1}\right) \partial^{\mu}\left(\delta \phi_{1}\right)(1+\epsilon)+\frac{1}{2} \partial_{\mu}\left(\delta \phi_{2}\right) \partial^{\mu}\left(\delta \phi_{2}\right)(1+\epsilon)+\epsilon \partial_{\mu}\left(\delta \phi_{1}\right) \partial^{\mu}\left(\delta \phi_{2}\right) \\
+\frac{3}{8} \sum_{i} \frac{\partial^{\mu}\left(\delta \tau_{i}\right) \partial_{\mu}\left(\delta \tau_{i}\right)}{\mathcal{V} \sqrt{\left\langle\tau_{i}\right\rangle}}-\frac{3 \sqrt{3}}{2 \sqrt{2}} \sum_{i} \frac{\sqrt{\left\langle\tau_{i}\right\rangle}}{\mathcal{V}} \partial^{\mu}\left(\delta \phi_{2}\right) \partial_{\mu}\left(\delta \tau_{i}\right)(1+\epsilon)+\frac{9}{4} \sum_{j>i} \frac{\sqrt{\left\langle\tau_{i}\right\rangle\left\langle\tau_{j}\right\rangle}}{\mathcal{V}^{2}} \partial^{\mu}\left(\delta \tau_{i}\right) \partial_{\mu}\left(\delta \tau_{j}\right) .
\end{gathered}
$$

Therefore we realise that there is no mixing between $\delta \phi_{1}$ and any of the small blow-up modes. As we pointed out at the beginning of this section, we notice that the structure of the mixing between the small moduli has the same form as the case of BI studied before.

\section{A.2.2 Canonical normalisation with loop corrections}

In this section we shall show how the introduction of string loop corrections to the scalar potential (A.103), gives a mass to $\delta \phi_{1}$ and introduces a coupling of this mode to the gauge bosons living on a stack of $D 7$-branes wrapping a blow-up cycle. More precisely string loops will produce a subleading correction in the canonical normalisation (A.112) such that the term:

$$
\left(\frac{1}{2} \frac{\partial^{\mu}\left(\delta \tau_{1}\right)}{\left\langle\tau_{1}\right\rangle}+\frac{\partial^{\mu}\left(\delta \tau_{2}\right)}{\left\langle\tau_{2}\right\rangle}\right)=\sqrt{\frac{3}{2}} \partial^{\mu}\left(\delta \phi_{2}\right)(1+\epsilon)
$$

in the kinetic Lagrangian (A.115), will not depend on just $\delta \phi_{2}$ anymore but a subleading dependence on $\delta \phi_{1}$ will also be introduced, generating a mixing between $\delta \phi_{1}$ and any blow-up mode present in the theory. Let us start from the potential:

$$
V=J\left[\frac{-(\ln \mathcal{V})^{3 / 2}+\tilde{\xi}}{\mathcal{V}^{3}}+\frac{a_{1}}{\mathcal{V}^{3} \sqrt{\tau_{1}}}\right], \quad \text { with } \quad a_{1} \simeq \frac{\mathcal{O}(1)}{J} \simeq \mathcal{O}(1)\left(g_{s} \ln \mathcal{V}\right)^{3 / 2}
$$

where we have schematically introduced the leading order dependence on the $g_{s}$ corrections. The way we shall proceed in order to work out the loop corrections to the canonical normalisation is the following one:

1. We shall still consider the inverse of the tree-level Kähler metric given by (A.104) but now with a loop corrected scalar potential (A.117);

2. We shall evaluate the matrix $M_{i k}^{2}=K_{i j}^{-1} V_{j k}$ (where now $V_{j k}$ are second derivatives of (A.117)) not at A.106) but at the loop corrected solution for $\tilde{\xi}$;

3. We can obtain the loop corrected solution for $\tilde{\xi}$ by noticing that the $g_{s}$ corrections, besides introducing in the scalar potential a dependence on $\tau_{1}$, introduce also a subleading dependence on $\mathcal{V}$. Therefore the volume minimum will acquire a tiny $\tau_{1}$-dependent shift $\mathcal{V}=\mathcal{V}_{0}+\delta \mathcal{V}\left(\tau_{1}\right)$ 
which has already been derived in appendix A.1 of 21]. We can use that result, plug the loop corrected expression for the volume in (A.106) and then obtain the loop corrected result for $\tilde{\xi}$ by Taylor expansion. Following this procedure, we obtain:

$$
\tilde{\xi}=\tilde{\xi}_{0}+\frac{3 a_{1}}{\sqrt{\left\langle\tau_{1}\right\rangle}}
$$

where with $\tilde{\xi}_{0}$ we have denoted the leading order solution given by (A.106).

4. We will assume that $\tau_{1}$ is stabilised by the remaining 1-loop terms in the potential which we have neglected since we are just interested in the leading order volume scaling of the corrections to canonical normalisation (A.112).

The new eigenvalues with the corresponding eigenvectors turn out to be:

$$
\begin{array}{lll}
m_{1}^{2} \simeq J \frac{a_{1}}{\mathcal{V}^{3} \sqrt{\left\langle\tau_{1}\right\rangle}} \sim \frac{\mathcal{O}(1)}{\mathcal{V}^{3} \sqrt{\left\langle\tau_{1}\right\rangle}} & \longleftrightarrow & \vec{v}_{1}=\left(\begin{array}{c}
-\frac{2\left\langle\tau_{1}\right\rangle}{\left\langle\tau_{2}\right\rangle} x(1+\delta) \\
x
\end{array}\right), \\
m_{2}^{2} \simeq J \frac{\sqrt{\ln \mathcal{V}}}{\mathcal{V}^{3}} \sim \frac{\mathcal{O}(1)}{g_{s}^{3 / 2} \mathcal{V}^{3} \ln \mathcal{V}} \quad \longleftrightarrow \quad \vec{v}_{2}=\left(\begin{array}{c}
\frac{\left\langle\tau_{1}\right\rangle}{\left\langle\tau_{2}\right\rangle} y(1+\delta) \\
y
\end{array}\right),
\end{array}
$$

where $\delta=\mathcal{O}\left(\frac{1}{\sqrt{\left\langle\tau_{1}\right\rangle} \sqrt{\ln \mathcal{V}}}\right) \ll 1$ since $\left\langle\tau_{1}\right\rangle=c \mathcal{V}^{2 / 3}$, and we have neglected corrections proportional to $\epsilon$ since $\epsilon \ll \delta$. We see from (A.119) that now $\delta \phi_{1}$ acquires a mass of the order $m_{1}^{2} \simeq c^{-1 / 2} \mathcal{V}^{-10 / 3}$. The values of $x$ and $y$ are now given by $x=y / \sqrt{2}=2\left\langle\tau_{2}\right\rangle(1+\delta) / \sqrt{6}$ and so the leading order loop-corrected canonical normalisation around the minimum looks like:

$$
\left\{\begin{array}{c}
\frac{\delta \tau_{1}}{\left\langle\tau_{1}\right\rangle}=-\left[\frac{2}{\sqrt{3}}(1+\delta / 3)\right] \delta \phi_{1}+\left[\sqrt{\frac{2}{3}}(1+2 \delta / 3)\right] \delta \phi_{2}, \\
\frac{\delta \tau_{2}}{\left\langle\tau_{2}\right\rangle}=\left[\frac{1}{\sqrt{3}}(1-2 \delta / 3)\right] \delta \phi_{1}+\left[\sqrt{\frac{2}{3}}(1-\delta / 3)\right] \delta \phi_{2} .
\end{array}\right.
$$

In terms of this new canonical normalisation, the expression (A.116) now reads:

$$
\left(\frac{1}{2} \frac{\partial^{\mu}\left(\delta \tau_{1}\right)}{\left\langle\tau_{1}\right\rangle}+\frac{\partial^{\mu}\left(\delta \tau_{2}\right)}{\left\langle\tau_{2}\right\rangle}\right)=\sqrt{\frac{3}{2}} \partial^{\mu}\left(\delta \phi_{2}\right)+\frac{\delta}{\sqrt{3}} \partial^{\mu}\left(\delta \phi_{1}\right) \sim \mathcal{O}(1) \partial^{\mu}\left(\delta \phi_{2}\right)+\mathcal{O}\left(\frac{c^{-1 / 2}}{(\ln \mathcal{V})^{1 / 2} \mathcal{V}^{1 / 3}}\right) \partial^{\mu}\left(\delta \phi_{1}\right),
$$

introducing a subleading mixing between $\delta \phi_{1}$ and any blow-up mode in the theory. Therefore, for the geometric regime case, the final canonical normalisation will look like (A.20)-(A.22) but now with a subleading dependence on $\delta \phi_{1}$ of the form (for the case of two blow-up modes $\tau_{3}$ and $\tau_{4}$ ):

$$
\begin{aligned}
\delta \tau_{1} & \sim \sum_{i=1}^{2} \mathcal{O}\left(\mathcal{V}^{2 / 3}\right) \delta \phi_{i}+\sum_{j=3}^{4} \mathcal{O}\left(\mathcal{V}^{1 / 6}\right) \delta \phi_{j} \sim \sum_{i=1}^{2} \mathcal{O}\left(\mathcal{V}^{2 / 3}\right) \delta \phi_{i} \\
\delta \tau_{2} & \sim \sum_{i=1}^{2} \mathcal{O}\left(\mathcal{V}^{2 / 3}\right) \delta \phi_{i}+\sum_{j=3}^{4} \mathcal{O}\left(\mathcal{V}^{1 / 6}\right) \delta \phi_{j} \sim \sum_{i=1}^{2} \mathcal{O}\left(\mathcal{V}^{2 / 3}\right) \delta \phi_{i} \\
\delta \tau_{3} & \sim \mathcal{O}\left(c^{-1 / 2} \mathcal{V}^{-1 / 3}\right) \delta \phi_{1}+\mathcal{O}(1) \delta \phi_{2}+\mathcal{O}\left(\mathcal{V}^{1 / 2}\right) \delta \phi_{3}+\mathcal{O}\left(\mathcal{V}^{-1 / 2}\right) \delta \phi_{4} \sim \mathcal{O}\left(\mathcal{V}^{1 / 2}\right) \delta \phi_{3} \\
\delta \tau_{4} & \sim \mathcal{O}\left(c^{-1 / 2} \mathcal{V}^{-1 / 3}\right) \delta \phi_{1}+\mathcal{O}(1) \delta \phi_{2}+\mathcal{O}\left(\mathcal{V}^{-1 / 2}\right) \delta \phi_{3}+\mathcal{O}\left(\mathcal{V}^{1 / 2}\right) \delta \phi_{4} \sim \mathcal{O}\left(\mathcal{V}^{1 / 2}\right) \delta \phi_{4}
\end{aligned}
$$

Similar considerations apply also for the case at the quiver locus.

\section{A.2.3 Moduli couplings}

The moduli couplings for the case of FI have the same behaviour as in the case of BI since the canonical normalisation has the same structure. The only difference is for the large modulus $\delta \phi_{1}$ 
since $\delta \phi_{2}$ behaves exactly as the large overall volume mode of the previous case. Hence we shall just focus on the couplings and decay rates of $\delta \phi_{1}$. As we have stressed before, the largest couplings are to visible and hidden gauge bosons, and on top of this, $\delta \phi_{1}$ is so light that it can decay only to those particles. Hence we need just to derive the strength of these interactions.

Assuming that $\tau_{3}$ is supporting a hidden sector while $\tau_{4}$ the visible sector, the fact that (A.124) and (A.125) have the same volume dependence on $\delta \phi_{1}$, implies that $\delta \phi_{1}$ couples to hidden gauge bosons $X_{3}$ and visible gauge bosons $g \equiv X_{4}$ with the same strength:

$$
\mathcal{L}_{\delta \phi_{1} X_{i} X_{i}} \sim\left(\frac{c^{-1 / 2}}{40(\ln \mathcal{V})^{1 / 2} \mathcal{V}^{1 / 3}}\right) \frac{\delta \phi_{1}}{M_{P}} G_{\mu \nu}^{(i)} G_{(i)}^{\mu \nu}, \quad i=3,4 .
$$

In addition, also $\tau_{1}$ and $\tau_{2}$ are wrapped by the stacks of $D 7$-branes which source the string loop corrections that stabilise the flat direction orthogonal to the overall volume. Thus each of them also supports a hidden sector. The fact that (A.122) and (A.123) have the same volume dependence on $\delta \phi_{1}$, implies that $\delta \phi_{1}$ couples to hidden gauge bosons $X_{1}$ and $X_{2}$ with the same strength:

$$
\mathcal{L}_{\delta \phi_{1} X_{i} X_{i}} \sim\left(\frac{1}{4 M_{P}}\right) \delta \phi_{1}\left(G_{(i)}^{h i d}\right)_{\mu \nu}\left(G_{(i)}^{h i d}\right)^{\mu \nu}, \quad i=1,2 .
$$

The decay rates corresponding to the couplings (A.126) are:

$$
\Gamma_{\delta \phi_{1} \rightarrow g g} \sim \Gamma_{\delta \phi_{1} \rightarrow X_{3} X_{3}} \sim\left(\frac{1}{6400 \pi \ln \mathcal{V}\left\langle\tau_{1}\right\rangle}\right) \frac{m_{1}^{3}}{M_{P}^{2}} \sim 5 \cdot 10^{-5} \frac{M_{P}}{c^{7 / 4}(\ln \mathcal{V}) \mathcal{V}^{17 / 3}},
$$

whereas the decay rates corresponding to the couplings (A.127) read:

$$
\Gamma_{\delta \phi_{1} \rightarrow X_{1} X_{1}} \sim \Gamma_{\delta \phi_{1} \rightarrow X_{2} X_{2}} \sim\left(\frac{1}{64 \pi}\right) \frac{m_{1}^{3}}{M_{P}^{2}} \sim 5 \cdot 10^{-3} \frac{M_{P}}{c^{3 / 4} \mathcal{V}^{5}},
$$

and so we conclude that $\delta \phi_{1}$ decays to $X_{1}$ and $X_{2}$ instead of $g$ and $X_{3}$ since:

$$
\frac{\Gamma_{\delta \phi_{1} \rightarrow g g}}{\Gamma_{\delta \phi_{1} \rightarrow X_{1} X_{1}}} \sim\left(\frac{10^{-2}}{\ln \mathcal{V}}\right) \frac{1}{c \mathcal{V}^{2 / 3}}=\left(\frac{10^{-2}}{\ln \mathcal{V}}\right) \frac{1}{\left\langle\tau_{1}\right\rangle} \ll 1
$$

We finally point out that when $\tau_{4}$ shrinks to zero size, the coupling of $\delta \phi_{1}$ to visible gauge bosons is even more suppressed since it scales as $c^{-1 / 2} /\left(M_{P} \mathcal{V}^{5 / 6}\right)$.

\section{References}

[1] E. Komatsu et al., arXiv:1001.4538 [astro-ph.CO].

[2] A. Mazumdar and J. Rocher, arXiv:1001.0993 [hep-ph].

[3] R. Allahverdi, R. Brandenberger, F. Y. Cyr-Racine and A. Mazumdar, arXiv:1001.2600 [hep-th].

[4] B. Fields and S. Sarkar, astro-ph/0601514; R. H. Cyburt, Phys. Rev. D 70 (2004) 023505 [astro-ph/0401091].

[5] R. Allahverdi, K. Enqvist, J. Garcia-Bellido and A. Mazumdar, Phys. Rev. Lett. 97 (2006) 191304 [hep-ph/0605035]; R. Allahverdi, A. Kusenko and A. Mazumdar, JCAP 0707 (2007) 018 [hep-ph/0608138]; R. Allahverdi, K. Enqvist, J. Garcia-Bellido, A. Jokinen and A. Mazumdar, JCAP 0706 (2007) 019 [hep-ph/0610134].

[6] K. Enqvist and A. Mazumdar, Phys. Rept. 380 (2003) 99 [hep-ph/0209244]; M. Dine and A. Kusenko, Rev. Mod. Phys. 76 (2004) 1 [hep-ph/0303065].

[7] R. Allahverdi, B. Dutta and A. Mazumdar, Phys. Rev. Lett. 99 (2007) 261301 [arXiv:0708.3983 [hep-ph]]; R. Allahverdi, B. Dutta and A. Mazumdar, Phys. Rev. D 75 (2007) 075018 [hep-ph/0702112]. 
[8] S. Gukov, C. Vafa and E. Witten, Nucl. Phys. B 584 (2000) 69 [Erratum-ibid. B 608 (2001) 477] [hep-th/9906070].

[9] K. Dasgupta, G. Rajesh and S. Sethi, JHEP 9908 (1999) 023 [hep-th/9908088].

[10] S. B. Giddings, S. Kachru and J. Polchinski, Phys. Rev. D 66 (2002) 106006 [hep-th/0105097].

[11] S. Kachru, R. Kallosh, A. Linde and S. P. Trivedi, Phys. Rev. D 68 (2003) 046005 [hep-th/0301240].

[12] V. Balasubramanian, P. Berglund, J. P. Conlon and F. Quevedo, JHEP 0503 (2005) 007 [hep-th/0502058].

[13] M. Cicoli, J. P. Conlon and F. Quevedo, JHEP 0810 (2008) 105 [arXiv:0805.1029 [hep-th]].

[14] J. P. Conlon, F. Quevedo and K. Suruliz, JHEP 0508 (2005) 007 [hep-th/0505076]; J. P. Conlon, S. S. Abdussalam, F. Quevedo and K. Suruliz, JHEP 0701 (2007) 032 [arXiv:hep-th/0610129].

[15] J. P. Conlon, A. Maharana and F. Quevedo, JHEP 0905 (2009) 109 [arXiv:0810.5660 [hep-th]]; R. Blumenhagen, J. P. Conlon, S. Krippendorf, S. Moster and F. Quevedo, JHEP 0909 (2009) 007 [arXiv:0906.3297 [hep-th]].

[16] A. Mazumdar, S. Panda and A. Perez-Lorenzana, Nucl. Phys. B 614 (2001) 101 [hep-ph/0107058]; N. Barnaby, C. P. Burgess and J. M. Cline, JCAP 0504 (2005) 007 [hep-th/0412040]; A. Mazumdar and H. Stoica, Phys. Rev. Lett. 102 (2009) 091601 [arXiv:0807.2570 [hep-th]]; R. H. Brandenberger, K. Dasgupta and A. C. Davis, Phys. Rev. D 78 (2008) 083502 [arXiv:0801.3674 [hep-th]].

[17] L. Kofman and P. Yi, Phys. Rev. D 72 (2005) 106001 [hep-th/0507257]; A. R. Frey, A. Mazumdar and R. C. Myers, Phys. Rev. D 73 (2006) 026003 [hep-th/0508139]. X. Chen and S. H. Tye, JCAP 0606 (2006) 011 [hep-th/0602136].

[18] D. R. Green, Phys. Rev. D 76 (2007) 103504 [arXiv:0707.3832 [hep-th]].

[19] N. Barnaby, J. R. Bond, Z. Huang and L. Kofman, JCAP 0912 (2009) 021 [arXiv:0909.0503 [hep-th]].

[20] J. P. Conlon and F. Quevedo, JHEP 0601 (2006) 146 [hep-th/0509012].

[21] M. Cicoli, C. P. Burgess and F. Quevedo, JCAP 0903 (2009) 013 [arXiv:0808.0691 [hep-th]].

[22] C. P. Burgess, J. M. Cline, H. Stoica and F. Quevedo, JHEP 0409 (2004) 033 [hep-th/0403119]; R. Kallosh and A. Linde, JHEP 0412 (2004) 004 [hep-th/0411011]; J. J. Blanco-Pillado, R. Kallosh and A. Linde, JHEP 0605 (2006) 053 [hep-th/0511042]; R. Kallosh and A. Linde, JHEP 0702 (2007) 002 [hep-th/0611183].

[23] C. Burgess, M. Cicoli, M. Gomez-Reino, F. Quevedo, G. Tasinato and I. Zavala, arXiv:1005.4840 [hep-th].

[24] A. Collinucci, M. Kreuzer, C. Mayrhofer and N. O. Walliser, JHEP 0907 (2009) 074 [arXiv:0811.4599 [hep-th]].

[25] M. Cicoli, M. Kreuzer and C. Mayerhofer, in preparation

[26] M. Berg, M. Haack and B. Kors, JHEP 0511, 030 (2005) [hep-th/0508043]; M. Berg, M. Haack and E. Pajer, arXiv:0704.0737 [hep-th]; M. Cicoli, J. P. Conlon and F. Quevedo, JHEP 0801 (2008) 052 [arXiv:0708.1873 [hep-th]].

[27] R. Blumenhagen, S. Moster and E. Plauschinn, JHEP 0801 (2008) 058 [arXiv:0711.3389 [hep-th]].

[28] T. W. Grimm, S. Krause and T. Weigand, arXiv:0912.3524 [hep-th].

[29] J. P. Conlon, JHEP 0605 (2006) 078 [hep-th/0602233].

[30] J. P. Conlon and D. Cremades, Phys. Rev. Lett. 99 (2007) 041803 [hep-ph/0611144].

[31] G. Aldazabal, L. E. Ibanez and F. Quevedo, JHEP 0002 (2000) 015 [hep-ph/0001083].

[32] J. P. Conlon, R. Kallosh, A. D. Linde and F. Quevedo, JCAP 0809 (2008) 011 [arXiv:0806.0809 [hep-th]].

[33] J. P. Conlon and F. G. Pedro, arXiv:1003.0388 [hep-th].

[34] K. S. Choi, I. W. Kim and J. E. Kim, JHEP 0703 (2007) 116 [hep-ph/0612107]. 
[35] R. Blumenhagen, M. Cvetic and T. Weigand, Nucl. Phys. B 771 (2007) 113 [hep-th/0609191];

L. E. Ibanez and A. M. Uranga, JHEP 0703 (2007) 052 [hep-th/0609213].

[36] O. Lebedev, C. Papineau and M. Postma, Phys. Lett. B 684 (2010) 154 [arXiv:0907.4630 [hep-th]].

[37] G. N. Felder, J. Garcia-Bellido, P. B. Greene, L. Kofman, A. D. Linde and I. Tkachev, Phys. Rev. Lett. 87 (2001) 011601 [hep-ph/0012142].

[38] G. N. Felder, L. Kofman and A. D. Linde, Phys. Rev. D 59 (1999) 123523 [hep-ph/9812289].

[39] L. Kofman, A. D. Linde and A. A. Starobinsky, Phys. Rev. D 56 (1997) 3258 [hep-ph/9704452].

[40] J. H. Traschen and R. H. Brandenberger, Phys. Rev. D 42 (1990) 2491; L. Kofman, A. D. Linde and A. A. Starobinsky, Phys. Rev. Lett. 73 (1994) 3195 [hep-th/9405187]; Y. Shtanov, J. H. Traschen and R. H. Brandenberger, Phys. Rev. D 51 (1995) 5438 [hep-ph/9407247].

[41] R. Micha and I. I. Tkachev, Phys. Rev. Lett. 90 (2003) 121301 [hep-ph/0210202].

[42] K. Enqvist, S. Kasuya and A. Mazumdar, Phys. Rev. Lett. 89 (2002) 091301 [hep-ph/0204270]; K. Enqvist, S. Kasuya and A. Mazumdar, Phys. Rev. D 66 (2002) 043505 [hep-ph/0206272].

[43] A. Kusenko and M. E. Shaposhnikov, Phys. Lett. B 418 (1998) 46 [hep-ph/9709492]; K. Enqvist and J. McDonald, Nucl. Phys. B 538 (1999) 321 [hep-ph/9803380].

[44] R. Allahverdi and A. Mazumdar, JCAP 0610 (2006) 008 [hep-ph/0512227]; R. Allahverdi and A. Mazumdar, hep-ph/0505050.

[45] R. Allahverdi and A. Mazumdar, Phys. Rev. D 76 (2007) 103526 [hep-ph/0603244].

[46] R. Allahverdi and A. Mazumdar, JCAP 0708 (2007) 023 [hep-ph/0608296]; R. Allahverdi and A. Mazumdar, Phys. Rev. D 78 (2008) 043511 [arXiv:0802.4430 [hep-ph]].

[47] M. E. Peskin, hep-th/9702094; M. A. Shifman, Prog. Part. Nucl. Phys. 39 (1997) 1 [hep-th/9704114].

[48] D. S. Freed and E. Witten, hep-th/9907189.

[49] K. Choi and K. S. Jeong, JHEP 0608 (2006) 007 [hep-th/0605108].

[50] I. Affleck, M. Dine and N. Seiberg, Nucl. Phys. B 241 (1984) 493.

[51] O. Aharony, J. Sonnenschein, M. E. Peskin and S. Yankielowicz, Phys. Rev. D 52 (1995) 6157 [hep-th/9507013].

[52] J. P. Conlon and F. Quevedo, JCAP 0708 (2007) 019 [arXiv:0705.3460 [hep-ph]].

[53] K. Kohri, A. Mazumdar and N. Sahu, Phys. Rev. D 80 (2009) 103504 [arXiv:0905.1625 [hep-ph]].

[54] L. Anguelova, V. Calo and M. Cicoli, JCAP 0910 (2009) 025 [arXiv:0904.0051 [hep-th]].

[55] K. Enqvist, S. Kasuya and A. Mazumdar, Phys. Rev. Lett. 93 (2004) 061301 [hep-ph/0311224].

[56] M. Goodsell, J. Jaeckel, J. Redondo and A. Ringwald, JHEP 0911 (2009) 027 [arXiv:0909.0515 [hep-ph]].

[57] J. P. Conlon, D. Cremades and F. Quevedo, JHEP 0701 (2007) 022 [hep-th/0609180].

[58] A. Brignole, L. E. Ibanez and C. Munoz, hep-ph/9707209. 